\title{
Abstracts of presentations at the XXV Congress of the Italian Phytopathological Society (SIPaV)
}

\author{
September $16-18,2019$. University of Milan, Milano, Italy
}

\begin{abstract}
New multiplex loop mediated isothermal amplification (LAMP) based assays for the early sequence-specific detection of Dothistroma pini, Dothistroma septosporum and Lecanosticta acicola on pine needles
\end{abstract}

C. Aglietti ${ }^{1,3}$, P. Capretti ${ }^{1}$, L. Ghelardini ${ }^{1}$, G. Marchi ${ }^{1}$, I. Barnes ${ }^{2}$, C. Villari $^{3}$

\begin{abstract}
${ }^{1}$ Department of Agriculture, Food, Environment and Forestry (DAGRI), University of Florence, Piazzale delle Cascine 28, I-50144, Firenze (Italy); ${ }^{2}$ Department of Biochemistry, Genetics and Microbiology, Forestry and Agricultural Biotechnology Institute (FABI), University of Pretoria, Pretoria, South Africa; ${ }^{3}$ Warnell School of Forestry \& Natural Resources, University of Georgia, Athens, Georgia, United States. E-mail: chiara.aglietti@unifi.it
\end{abstract}

Dothistroma pini, D. septosporum and Lecanosticta acicola needle blights are among the most serious needle fungal diseases affecting pine species and are causing concern in many countries. Because management strategies might vary depending on the pathogen, a fast and accurate diagnosis is crucial. Current DNA-based assays available for their detection require well-equipped laboratories and are time consuming. The possibility to screen for such pathogens directly in the field could significantly expedite a rapid response to the threat. The objective of this work was to develop and validate three real-time monitoring loop mediated isothermal amplification (LAMP) assays that target the three pathogens using a portable device. LAMP primers and fluorescent assimilating probes were designed to recognize the beta tubulin ( $\beta$-tub2) and elongation factor $(E F-1 \alpha)$ regions for the two Dothistroma species, and L. acicola, respectively. Fifty-five different isolates from various geographical origins and belonging to the species phylogenetically closer to the three target ones were tested and results confirmed that the assays are highly specific. The assays showed high sensitiv-

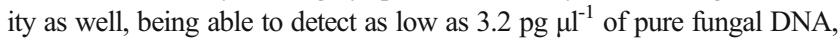
and to detect the pathogens in naturally infected needles in less than $30 \mathrm{~min}$. The simplicity, sensitivity, specificity, and minimum required equipment make these LAMP assays ideal for in-field routine plant tissue testing and could have great implications for the management of D. septosporum, $D$. pini and $L$. acicola both in nurseries and forest systems.

\section{Phytophthora species in rivers and streams of Eastern Sicily}

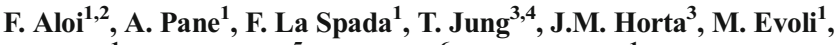
M. Riolo ${ }^{1}$, J.B. Ristaino ${ }^{5}$, L. Schena ${ }^{6}$, S.O. Cacciola ${ }^{1}$

${ }^{1}$ Department of Agricultural, Food and Environment (Di3A), University of Catania, via S. Sofia 100, 95123 Catania, Italy; ${ }^{2}$ Department of Agricultural, Food and Forest Sciences, University of Palermo, Viale delle
Scienze, Building 4, 90128 Palermo, Italy; ${ }^{3}$ Phytophthora Research Center Mendel University, Brno, Czech Republic; ${ }^{4}$ Phytophthora Research and Consultancy, Nußdorf, Germany; ${ }^{5}$ Department of Plant Pathology, North Carolina State University, P.O. Box 7616, Raleigh, NC 27695-7616, USA; ${ }^{6}$ Dipartimento di Agraria, University Mediterranea of Reggio Calabria, Feo di Vito s.n.c., 89122 Reggio Calabria, Italy.E-mail:olgacacciola@unict.it

Phytophthora species were isolated from rivers and streams in eastern Sicily by in situ baiting using floating mesh bag-styrofoam rafts and identified by sequence analysis of internal transcribed spacer (ITS) ribosomal DNA (rDNA). The aim of this study was to investigate the ecological diversity, expressed as Shannon Diversity Index (SDI), of Phytophthora populations from river systems running nine Protected Natural Areas typical of Sicilian riparian plant communities. The major waterways examined included the Alcantara, Anapo and Fiumefreddo rivers on the eastern coast of Sicily, and streams with torrential water regimes such as Sciambro, on mount Etna. The most prevalent species identified in rivers and streams were $P . \times$ cambivora, $P$. citrophthora, $P$. frigida, $P$. gonapodyides, $P$. hydropathica, $P$. sp. kelmania, $P$. lacustris, $P$. multivora, $P$. plurivora, $P$. polonica, $P$. pseudocryptogea and P. thermophila. High SDI values and a significant proportion of exotic Phytophthora species were found in 50\% of the examined river systems, particularly in systems highly disturbed by human activities (e.g. the Fiumefreddo river, a small watercourse in a small natural reserve recently established in a highly anthropized area). By contrast, the naturalized systems showed the lowest diversity (e.g. the Sciambro stream, a seasonal water course at high altitude in the Mount Etna regional park, crossing a single-tree-species forest of black pine).

${ }^{1}$ H-NMR - based metabolomic approach on Xylella fastidiosa subsp. pauca-infected olive trees: disease biomarkers and a field treatment response characterization

F. Angilè ${ }^{1}$, C.R. Girelli ${ }^{1}$, L. Del Coco ${ }^{1}$, D. Migoni ${ }^{1}$, L. Zampella $^{2}$, M. Scortichini $^{2}$, F.P. Fanizzi ${ }^{1}$

${ }^{1}$ Department of Biological and Environmental Sciences and Technologies, University of Salento, Prov.le Lecce-Monteroni, I-73100 Lecce, Italy; ${ }^{2}$ Council for Agricultural Research and EconomicsResearch Centre for Olive, Fruit Trees and Citrus, Via Torrino, 3, I-81100, Caserta, Italy. E-mail: federica.angile@unisalento.it

Xylella fastidiosa subsp. pauca is a xylem-limited bacterial phytopathogen associated on many hectares with the "olive quick decline syndrome" in the Apulia region (southern Italy). In a previous work, in an attempt to find out possible compounds showing the capacity to reduce the 
concentration of $X$. fastidiosa subsp. pauca within olive leaves, we tested a zinc-copper-citric acid biocomplex on symptomatic sensitive cultivars Ogliarola salentina and Cellina di Nardò olive trees. Through ${ }^{1} \mathrm{H}$ NMR and multivariate statistical analysis, we assessed the metabolomic profiles of extracts from the leaves of the two cultivars, observing an opposite trend concerning the sugars and polyphenols content. In the present study, the ${ }^{1} \mathrm{H}-\mathrm{NMR}$-based metabolomic approach was used to provide a snapshot of the plant-pathogen interaction that modifies the plant metabolic pathways, and to identify potential biomarkers of $X$. fastidiosa subsp. pauca infection to olive trees at its early stages. In both Ogliarola salentina and Cellina di Nardò cultivars, metabolites such as quinic acid, the aldehydic form of oleoeuropein, ligustroside and phenolic compounds were consistently found as discriminative for the untreated trees in comparison with treated trees. Quinic acid was confirmed as a disease biomarker for the olive trees infected by $X$. fastidiosa subsp. pauca. This study showed how $X$. fastidiosa subsp. pauca infection strongly modifies the overall metabolism of olive trees, and how a zinc-copper-citric acid biocomplex can induce an early re-programming of the metabolic pathways in the infected trees. These results could be useful for the development of strategies for infection containment and plant health promotion.

\section{Factors affecting outbreaks of Heterobasidion on conifers and broad-leaves in the area of the Serre Calabresi}

\section{N. Anselmi ${ }^{1}$, P. Gonthier ${ }^{2}$, M.R. Pupo ${ }^{1}$, G. Contabile ${ }^{3}$}

${ }^{1}$ Department of Innovation in Biological, Agro-food and Forest systems (DIBAF), University of Tuscia, Via S. Camillo De Lelli, snc., 01100 Viterbo; ${ }^{2}$ Department of Agricultural, Forest and Food Sciences, University of Torino, Largo P. Braccini 2, 10095 Grugliasco, Torino; ${ }^{3}$ Forestry Doctor, Serra San Bruno, Vibo Valentia.E-mail: anselmicasa@live.it

The genus Heterobasidion includes some of the most harmful pathogens of conifers worldwide. In the last decades, in Calabria, the fungus has been found with increasing frequencies on silver fir (Abies alba), but also on broadleaves, elsewhere rarely affected. The purpose of this work was to identify the fungus associated with different tree species at the species level, and to monitor the evolution of outbreaks over time in relation to climatic trends and forest tree species composition. Based on the outcomes of TSCPPCR assays, Heterobasidion abietinum Niemelä \& Korhonen was found exclusively on silver fir, while Heterobasidion annosum (Fr.) Bref. was found on beech (Fagus sylvatica), black pine (Pinus nigra laricio) and Douglas fir (Pseudotsuga menziesii). The greatest damages were detected on silver fir and beech, while they were occasional on Douglas fir and rare on pine. Based on the monitoring of silver fir and beech every six months in three different areas over a ten year period, disease severity appeared affected by rainfall, with an increase in the decline of trees after periods of severe drought and a slowdown with well-distributed rainfall during the year. Outbreaks were strongly slowed down in mixed forests in relation to an increase in the presence of nonsusceptible species. Outbreaks of Heterobasidion in Calabria might be linked to climate change leading to long periods of drought in the last decades, and affecting especially tree species at the edge of their natural range. Tree species diversity is expected to minimize the risk of outbreaks.

\section{Identification and characterization of tomato root bacteri- al endophytes for integrated disease control program}

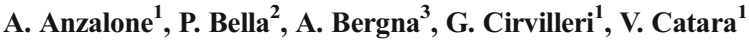 \\ ${ }^{1}$ Dipartimento di Agricoltura Alimentazione e Ambiente, Università degli \\ Studi di Catania, Via Santa Sofia 100, 95123 Catania, Italia;
}

${ }^{2}$ Dipartimento di Scienze, Agrarie, Alimentari e Forestali, Università degli Studi di Palermo, Viale delle Scienze, 90128 Palermo, Italia; ${ }^{3}$ Institute of Environmental Biotechnology, Graz University of Technology, Via Petersgasse 12, 8010 Graz, Austria.E-mail:vcatara@unict.it

In the last decade the need of novel methodologies for chemicalsfree production of tomato plants has been extensively addressed. Among the most promising biocontrol agents, endophytic bacteria received a great attention due to their intimate and non-detrimental association with plant tissues and to their capability to colonise internal host tissues. In the framework of a population study on root-associated bacteria of tomatoes grown in soil in the greenhouse environment in Sicily we set up a collection of 106 bacterial endophytes. They were investigated for a number of plant growth promoting (PGP) and tomato phytopathogen control traits in order to select potential bacterial strains to be further evaluated as bioinoculants. A high number of bacterial strains (38 out of 106) showed in vitro antagonistic activity against all the phytopathogens tested, namely the bacteria Clavibacter michiganesis pv. michiganensis, Pseudomonas syringae pv. tomato, P. corrugata, Xanthomonas euvesicatoria pv. perforans, and the fungus Fusarium oxysporum f. sp. lycopersici. Nevertheless, all the strains showed antagonistic activity at least to one of the microorganism tested, although to a different extent. Further characterisation showed that $28.3 \%$ of strains were able to produce siderophores, $50.94 \%$ were able to solubilise phosphates, and $68.86 \%$ showed resistance to salinity $(\mathrm{NaCl} 8 \%)$. Identification at species/genus level of 87 endophytic strains was conducted by $16 \mathrm{~S} r R N A$ gene sequencing. The sequences were searched against NCBI nucleotide database using BLASTn. Taxonomy information was assigned according to the highest score sequence. Bacterial isolates belonged to Gammaproteobacteria and Bacilli classes covering 23 distinct species belonging to six genera.

\section{In vitro efficacy of chemical and biological fungicides against Diaporthe growth}

\section{R. Arciuolo ${ }^{1}$, G. Chiusa ${ }^{1}$, N. Spigolon ${ }^{2}$, G. Castello ${ }^{2}$, P. Battilani ${ }^{1}$}

${ }^{1}$ Università Cattolica del Sacro Cuore di Piacenza, Department of Sustainable Crop Production (DI.PRO.VE.S.), Via Emilia Parmense 84, 29122 Piacenza ; ${ }^{2}$ SOREMARTEC ITALIA S.r.l., Piazzale Pietro Ferrero 1, Alba (CN), Italy.E-mail: roberta.arciuolo@unicatt.it

Diaporthe spp. was recently identified as the causal agent of brown spots on hazelnuts kernels and internal browning, visible after nut half cut, causing non-compliance to quality standard. These defects show incidence variable between 1-2 and $15 \%$, depending on weather conditions and growing areas. Actions are under consideration, including fungicide application, in order to improve kernel quality. The present study was designed to evaluate in vitro the efficacy of chemical and biological active ingredients in reducing Diaporthe mycelial growth, as preliminary approach for field trials. D. eres isolated from hazelnuts was used for the study. Chemicals, including Kresoxim-methyl, Boscalid+Kresoximmethyl, Fluxapyroraxad+Difenoconazole, Dithianon and Dithianon+ Potassium phosphonate and biocontrol agents (BCA) Bacillus amyloliquefaciens, B. subtilis QST713 (two formulations), Streptomyces griseoviridis K61 and Trichoderma gamsii + T. asperellum were used. Media amended with chemicals (four concentrations 1-1000 $\mathrm{ppm}$ ) and dual cultures for BCAs were considered; trial were managed in triplicate. Inoculated dishes were incubated at three temperatures $(\mathrm{T}=15$, $20,25^{\circ} \mathrm{C}$ ) and the percentage of growth inhibition was computed up to 
21 days of incubation. Fluxapyroraxad+Difenoconazole showed the strongest effect on Diaporthe growth (mean reduction $=72 \%$ ) while Kresoxim-methyl was ineffective. Concerning BCAs, B. subtilis was the most effective, with a mean growth reduction $=92 \%$, followed by B. amyloliquefaciens (89\%) and S. griseoviridis -Trichoderma spp. $(60 \%)$. Chemicals performed better at $15^{\circ} \mathrm{C}$ and $\mathrm{BCAs}$ at $25^{\circ} \mathrm{C}$, with D. eres growth reduction of $26 \%$ and $81 \%$ respectively, as mean of all conditions considered. Based on these results, field trials are ongoing in Turkey to test the performances of selected compounds in reducing defects caused by Diaporthe in hazelnuts orchards.

\section{BABA-induced resistance after the discovery that plants produce $\mathrm{BABA}$ : where is the link?}

\author{
A. Balmer ${ }^{1}$, F. Stefanelli ${ }^{1}$, G. Glauser ${ }^{2}$, B. Mauch-Mani ${ }^{1}$, I. Baccelli ${ }^{1,3}$ \\ ${ }^{1}$ University of Neuchâtel, Institute of Biology, Rue Emile-Argand 11, \\ 2000, Neuchâtel, Switzerland; ${ }^{2}$ University of Neuchâtel, Neuchâtel \\ Platform of Analytical Chemistry (NPAC), Avenue de Bellevaux 51, \\ 2000, Neuchâtel, Switzerland; ${ }^{3}$ CNR-IPSP, Institute for Sustainable \\ Plant Protection, Via Madonna del Piano 10, 50019 Sesto Fiorentino \\ (FI).E-mail: ivan.baccelli@ipsp.cnr.it
}

$\beta$-aminobutyric acid (BABA) is well-known to the scientific community as a priming inducer, since it induces resistance by stimulating plants to respond faster and/or stronger to following stresses. BABA was considered absent in nature until two years ago, when we revealed its endogenous production by a number of plants, including Arabidopsis and some crops. Further analyses revealed that BABA levels increase following pathogen infection and abiotic stresses, while in non-stressed plants BABA levels gradually increase during senescence. Interestingly, the production of BABA is controlled by the plant immune system and is stimulated by PAMPs, DAMPs, and effectors. Infection with avirulent Pseudomonas syringae pv. tomato (Pst) DC3000 avrRpt2 leads to a faster increase of BABA than infection with virulent Pst DC3000. Therefore, our results overall suggest that endogenous BABA plays a role in defense. We have recently found that during leaf infection BABA is accumulated only locally, with no detectable increase occurring in non-infected systemic plant parts. This result seems thus to contrast with an expected role for endogenous BABA in priming, as suggested by the literature existing on priming by synthetic BABA. Nevertheless, we suggest how endogenous and synthetic BABA could prime plants in the light of our recent results. In 1), a local increase of BABA could lead to the generation of a still unknown mobile priming signal; in 2), a local increase of endogenous BABA could take part to a local defense response, and synthetic BABA would prime plants in an indirect way by "mimicking" a widespread defense.

\section{Evaluating the efficacy of a biological formulate against Flavescence dorée phytoplasma}

\section{Baldo, M. Calassanzio, G. Filippini, C. Ratti}

Department of Agricultural Sciences, Alma Mater Studiorum University of Bologna, Viale G. Fanin, 44, 40127, Bologna, Italy. E-mail: david.baldo2@unibo.it

Flavescence dorée (FD) phytoplasma disease is provoking serious losses in the wine industry every year. The only active compounds able to control phytoplasmas are the antibiotic oxytetracyclines. In this work, the first compound designed to act directly on the phytoplasmas was tested to evaluate its potentiality. The formulate, consisting of essential oils, micro-elements and MICROSAP (a drug-delivery molecule), was tested on periwinkle plants infected by FD phytoplasma with an ex-vivo approach. Exposing cuts from infected plants directly to the formulate solution, we evaluated the efficacy of the preparation. After the exposure for $48 \mathrm{~h}$ and 10 days, 114 slices were taken from cuts and analyzed by scanning electron microscope evaluating the phytoplasmas morphology on 60 phloem cells for each slice. A number of the observed slices were then processed for molecular analysis by ddRT-PCR (digital droplet RTPCR) for absolute quantification of phytoplasmas' nucleic acids. The results obtained showed a compromised morphology and significant reduction of phytoplasmas, both in term of number and replication ability, in the cuts treated with the new formulate. A similar effect was observed on slices from cuts treated with the tetracyclines. Furthermore, the use of scanning electron microscopy in parallel with molecular tools was proven to be efficient in verifying the efficacy of compounds against a pathogen impossible to be cultivated in axenic culture. Further studies will focus on the application of the compound directly on grapevine plants to test its efficacy in the field.

\section{Med-Berry - Developing new strategies to protect straw- berry crop in Mediterranean countries}

\section{E. Baraldi (THE MED-BERRY CONSORTIUM)}

DISTAL-UniBo, Viale Fanin 42, 40127, Bologna. E-mail: elena.baraldi@unibo.it

Med-berry is a PRIMA project coordinated by DISTAL-UniBo, aiming at the development of new protocols, tools and strategies to protect strawberry crop in Mediterranean countries. Strawberry is a fundamental crop for the Mediterranean area. Up to $26 \%$ of the world strawberries are produced and important strawberry industries and scientific community are active here. Strawberry fruits have unique flavour and precious nutritional properties and their economic value is extremely high. Growing healthy strawberries using sustainable protection solutions that preserve the fruit quality and yield, and respect human health and environment is a challenging task, specially under the temperature increase in Mediterranean basin. In this project complementary expertise of researchers from public and private institutions from Italy, Spain, France, Morocco and Turkey are joined in the effort to develop innovative tools, protocols and strategies suited to revise the pathogen control strategies in view of innovative concepts of crop protection management. To this, five WPs will focus on the application of conventional instruments (e.g. traditional breeding programs made with local germplasm), New Breeding Techniques (NBT) and biotech RNAi protocols to develop new plants and products that counteract the most aggressive pathogens and the new phytosanitary emergences. Med-Berry results will have impact both at level of environment, research, industries and society.

Postharvest tritrophic interaction involving biocontrol yeast, fungal pathogen and host fruit: a transcriptomic study

G. Barone ${ }^{1}$, D. Palmieri ${ }^{1}$, C. Miccoli ${ }^{1}$, F. De Curtis ${ }^{1}$, R. Castoria ${ }^{1}$, G. Ianiri $^{1,2}$

${ }^{1}$ Department of Agricultural, Environmental and Food Sciences, University of Molise, via F. De Sanctis snc, 86100 Campobasso, Italy. E-mail: castoria@unimol.it; ${ }^{2}$ Department of Molecular Genetics and Microbiology, Duke University Medical Center, 312 CARL Building, Research Drive, Durham, NC 27710,USA. E-mail: giuseppe.ianiri@duke.edu 
Biological and integrated control strategies based on biocontrol agents can address the need of lowering chemical inputs for crop defense. The study of the mechanisms underlying biocontrol activity is pivotal to make these strategies more effective. In this regard, omic approaches are invaluable for the insights they can provide. In this work, we studied the molecular interactions involving the biocontrol yeast Papiliotrema terrestris strain LS28 (BCA), the postharvest patulin-producing pathogen Penicillium expansum strain 7015, and apple fruits cv. Golden delicious. We performed RNAseq analysis during both their dual and tritrophic interactions to identify the differentially expressed genes (DEGs) of LS28 and/or $P$. expansum applied alone or in combination in apple wounds. For the BCA, gene ontology (GO) classification revealed that upregulated genes are involved in oxidation-reduction processes and transmembrane transport, while for $P$. expansum transcription and transmembrane transport were the most represented GO categories. Analysis of the transcriptomic changes of the host during interaction with the $\mathrm{BCA}$ and/or the fungus is in progress. This transcriptomic analysis combined with the recent development of transformation systems for LS28 allows the functional characterization of genes playing a crucial role for biocontrol activity, thus contributing to advance the knowledge on the molecular mechanisms that underlie biocontrol activity.

\section{Monitoring and preventing grapevine trunk diseases in the nursery: comparative assays to control the related pathogens in propagation material}

\section{E. Battiston ${ }^{1}$, S. Schiff ${ }^{2}$, G. Carella ${ }^{1}$, S. Moretti ${ }^{1}$, A. Pacetti ${ }^{1}$, C. Tani ${ }^{2}$, S. Di Marco ${ }^{3}$, L. Mugnai ${ }^{1}$}

${ }^{1}$ Dipartimento di Scienze e Tecnologie Agrarie, Alimentari, Ambientali e Forestali (DAGRI) - Sez. Patologia vegetale ed Entomologia, Università degli Studi di Firenze, P.le Cascine 28 - 50144 Firenze; ${ }^{2}$ Dipartimento di Biologia, Via P.A. Micheli, 3, 50121 Firenze, Italy; ${ }^{3}$ Istituto di Bioeconomia, IBE CNR, Via Gobetti 101 - 40129 - Bologna, Italy. Email:enrico.battiston@unifi.it

The control of grapevine trunk diseases (GTDs) represents a big challenge for grapevine nurseries due to the high infection risks on the propagating material. Plant material disinfection before storage and the grafting practice are both potential factors able to affect the future GTDs development in young vines in field. In this framework, this research is aimed to evaluate the microbiome (especially taking into account GTDs pathogens) in the propagation material undergone through different disinfection treatments, and to compare the impact of three grafting methods on the formation of an active and functional vascular system. To assess the first aim, the microbiome of the scion (cv. Glera) and rootstock (cv. Kober 5BB) (by DNA sequencing technologies and intelligent computing) was preliminary analyzed just before grafting, in order to detect differences on the fungal and bacterial community prior treatment. For the second aim, three methods of grafting, (i) Omega graft, (ii) Whip and Tongue graft and (iii) Full cleft graft, were compared by observing xylogenesis and vascular tissue formation at the grafting point, in order to detect the factors potentially influencing the vine physiology. Early results showed a distinct profile in fungi and bacteria for both rootstock and scion. In rootstock, a higher abundance of GTDs agents was detected, especially in the bark. The anatomical observation on grapevine cuttings, immediately after callusing, revealed differences between all the three grafting methods: the full cleft graft showed the most complete and homogenous callus, which is the ideal condition to avoid the presence of necrosis, potentially colonized by the GTDs pathogens.
Fatty acid metabolism in Fusarium verticillioides

\author{
M. Beccaccioli ${ }^{1}$, A. Cacciotti ${ }^{1}$, V. Scala ${ }^{2}$, M. Reverberi ${ }^{1}$
}

${ }^{1}$ Department of Enviromental Biology, "Sapienza" University of Rome, P.le Aldo Moro 5, 00185 Rome, Italy; ${ }^{2} C R E A-D C$, Via Carlo Giuseppe Bertero 22, 00156 Roma, Italy.E-mail:marzia.beccaccioli@uniroma1.it

Fatty acids (FA) are a metabolic energy source and are building blocks of membrane lipids; moreover, they play a role in the signaling among hosts and pathogens. The ascomycete Fusarium verticillioides is a pathogenic fungus of maize; it is a valuable organism for elucidating processes in eukaryotic cell such as fatty acid metabolism. Oxidized FA, i.e. the oxylipins, are important drivers of the host-pathogen interaction. Previous studies assessed that their synthesis appear tightly coordinated within the subset of oxylipin-related genes and with the genes controlling the synthesis of FA. We suggest here that the calcium-regulated transcription factor Crzl (orthologue in F. verticillioides Fvcrz 1) is probably involved in the upstream regulation of several genes related to fatty acid and oxylipin synthesis. Through a transcriptomic and metabolomics approach, we analyzed gene expression and FA synthesis in F. verticillioides wild type and mutant strain $\operatorname{crz} 1 \Delta$ under in vitro conditions. Notably, we evaluated the expression of genes related to the FA biosynthesis, transformation, and oxylipins synthesis. Transcriptomic approach was associated to a mass spectrometry analysis to quantify several lipids. Apparently and intriguingly, Crzl did not affect significantly the expression of FA and oxylipin-related genes but controlled the total amount of FA and oxylipin in F. verticillioides. In fact, FA and oxylipin amount is reduced significantly in crzl $\Delta$ compared to WT strain whilst gene expression resulted almost unscathed. Advances in understanding such processes in $F$. verticillioides will provide helpful insights to the study of lipid-mediated cross talk within fungus-plant interaction.

\section{Detection of fungal pathogens threatening the oak trees in a coastal forest}

M. Beccaccioli ${ }^{1,2}$, C. Fanelli ${ }^{1,2}$, S. Savini ${ }^{1}$, A. Grottoli ${ }^{2,3}$, V.E. Cambria $^{2,4}$, L. Scarnati ${ }^{5}$, M. Reverberi ${ }^{1,2}$

${ }^{1}$ Department of Enviromental Biology, "Sapienza" University of Rome, P.le Aldo Moro 5, 00185 Rome, Italy; ${ }^{2}$ SARA EnviMob srl, Via dei Castani 116 Roma, Italy; ${ }^{3} D A F N E$ - University of Tuscia, Via San Camillo de Lellis snc 01100, Viterbo, Italy; ${ }^{4}$ Department TESAF, University of Padova, Via dell'Università 16, 35020 Legnano (PD), Italy; ${ }^{5}$ ARSIAL-Via R. Lanciani 38, 00162 Rome, Italy. E-mail: marzia.beccaccioli@uniroma1.it

Coastal forests are among the most degraded and threatened ecosystems in the EU. Several habitats and species in the forest of Palo Laziale are vulnerable. Actions to improve the conservation of habitats are particularly needed in this ecosystem. The first signs of forest dieback were observed in 1995 and after the summer drought of 2003, dead tree have progressively increased up to $40 \%$ because of the presence of several pathogens, among which Biscogniauxia mediterranea. This ascomycete after a long endophytic phase may cause charcoal disease in oak trees.

Several interventions on the territory have been planned, including the reforestation. To produce pathogen-free oak seedlings, seeds were collected in several sites in Palo Laziale forest where the oak trees showed symptoms, and in a symptomless site. The collected seeds were divided in lots. A forestry nursery was equipped to grow the seeds harvested to use the seedlings for reforestation. After three months, we found different 
levels of growth according to the site of collection; the seeds from Palo Laziale produced smaller plants. DNA was extracted from the seeds of each lot to identify by ITS region (Internal Transcribed Region) amplification and sequencing possible pathogens.

We found DNA from several fungal species, supporting the idea that some fungal pathogens could be endophytic to seed. The seedlings of uninfected lots will be used for the reforestation.

This work was supported by LIFE17 NAT/GR/000511

\section{Shoot blight and crown dieback of Chamaecyparis lawsoniana in Tuscany}

\section{A. Benigno, P. Capretti, S. Moricca}

Department of Agricultural, Food, Environmental and Forestry Science and Technology (DAGRI), Plant Pathology and Entomology Division, University of Florence, Piazzale delle Cascine 28, 50144, Firenze, Italy. E-mail: salvatore.moricca@unifi.it

Wilting and dieback of crown portions were observed during winter on Chamaecyparis lawsoniana trees growing in hedges and in rows in a mountain area of the Tuscan Apennines (near Vallombrosa). Symptoms started with tip blights, gradually progressing inwards to older twigs and branches, down to the stem. The infected foliage changed colour, from straw yellow to reddish, to brown-gray ash. Two distinctive patterns of crown dieback were observed in two localities: a progressive wilt, with a patchy, more or less irregular distribution of disease in one locality; a uniform wilt with die-off of the lower crown (up to $1 \mathrm{~m}$ ) in the other locality. In both situations, dark acervuli erupted in spring (May-June) through the epidermis of necrotized tissues. Isolations on PDA in the laboratory gave two fungi from the two localities: Pestalotiopsis funerea was consistently associated with the irregular, patchy crown diebacks of trees; Kabatina thujae was isolated at high percentages (90\%) from necrotic shoots of trees with uniform dieback of the lower crown portions. These fungi are opportunistic or weak parasites of members of Cupressaceae, and both are known to have an endophytic lifestyle. However, under favourable conditions, they can cause significant damage. In the investigated area (about $1000 \mathrm{~m}$ of altitude), some environmental factors may have favoured infection by these agents: summer drought, that has weakened trees; the density of vegetation in hedges and rows, that may have prevented air circulation, favouring excess moisture; and frost damage, that may have created entry points into host's internal tissues.

Thousand Cankers Disease of walnut in Italy: epidemiological aspects of an emerging disease

\section{A. Benigno, T. Panzavolta, M. Bracalini, S. Moricca}

Department of Agricultural, Food, Environmental and Forestry Science and Technology (DAGRI), Plant Pathology and Entomology Division, University of Florence, Piazzale delle Cascine 28, 50144, Firenze, Italy. E-mail: salvatore.moricca@unifi.it

The fungus Geosmithia morbida (Ascomycota, Hypocreales, Bionectriaceae) and its insect vector, the walnut twig beetle (WTB) Pityophthorus juglandis (Coleoptera, Curculionidae), are responsible for Thousand Cankers Disease (TCD) of walnut, a lethal disease that has caused extensive die-off of walnut species in the southwest of the USA and in northern Mexico. In recent years TCD has been reported in Italy, the only country outside its native range were it has dispersed to date. Until recently, the disease was supposed to have occurred only in northern Italy, where it was thought to have been successfully eradicated. The recent report of TCD in Tuscany opens a new and worrying scenario about its possible spread southwards. The attention on this fungus/beetle complex should be very high for two main reasons: 1) the center and south of the Italian Peninsula host the largest walnut plantations; 2) the environmental factors of central and southern Italy are more similar to those of the area of origin of TCD, therefore, presumably, more conducive to disease. Preliminary investigations on the physiological requirements of the fungus and the bio-ecology of its insect vector confirmed the thermophilic nature of both organisms. Since climate models predict for the Mediterranean area reduced precipitation, increased mean temperatures, extreme droughts and hot waves in the coming years, it is easy to assume that the possible spread of WTB southwards could have devastating effects for the cultivation of walnut in our country.

Not only long-term sieve-element blockage against phytoplasmas: role(s) of callose in plant defence-mechanisms

\section{Bernardini, L. Pagliari, S. Buoso, A. Loschi, S. Santi, R. Musetti}

Department of Agricultural, Food, Environmental and Animal Sciences, University of Udine, via delle Scienze, 206 I-33100 Udine, Italy. E-mail: rita.musetti@uniud.it

Phytoplasmas are unculturable phloem-limited prokaryotes, affecting many crops of economic importance. Infection causes several modifications at cytological and physiological level, resulting in yield reduction. One of these modifications is the phloem plugging: several studies attested the importance of the sieve-element occlusion related (SEOR) proteins and callose, respectively in the brief- and long-term plugging of the sieve plates. Apart the role in the occlusion, callose has multiple biological functions: for example, it takes part in the processes of phloem maturation in intact plants, influencing the formation and development of the sieve-plate pores, so influencing the mass flow. The synthesis of callose requires several steps, so the existence of a callose synthase complex, interacting with different proteins, related to sucrose synthesis and metabolism, has been suggested. In a previous work, the anomalous deposition of callose in phytoplasmainfected phloem tissue and the altered modulation of two callose synthase genes have been described as only a small part of all the transcriptional modifications observed for the sucrose transport and metabolism following infection. With the aim to highlight the different roles of callose in phytoplasma-infected plants, especially those related to the plant early defense signaling, wild-type and AtCas7ko Arabidopsis plants were infected with phytoplasmas. Modifications at morphological and transcriptomic levels in the two lines were compared and discussed.

This work was funded by the University of Udine, Department of Agriculture, Food, Environment and Animal Sciences, Project Start-up PRID 2018

Insect-borne prokaryote-associated diseases in tropical and subtropical perennial crops (TROPICSAFE): improving the production of citrus, grapevine and coconut palms

\author{
A. Bertaccini ${ }^{1}$, K. Krüger ${ }^{2}$, N. Fiore ${ }^{3}$, A. Tena ${ }^{4}$, M. Nicolaisen ${ }^{5}$, L. \\ Cesaro $^{6}$ \\ ${ }^{1}$ Alma Mater Studiorum - University of Bologna, DISTAL, Bologna, \\ Italy; ${ }^{2}$ University of Pretoria, Department of Zoology and Entomology,
}


Pretoria, South Africa; ${ }^{3}$ University of Chile, Faculty of Agronomy, Department of Plant Pathology, Santiago, Chile; ${ }^{4}$ IVIA, Valencia, Spain; ${ }^{5}$ Aarhus University, Demnark; ${ }^{6}$ CREA, Italy

The project addresses insect-borne prokaryote-associated diseases of palm, citrus and grapevine in tropical and subtropical areas seriously affecting the trade and import of agricultural products and materials worldwide. These diseases are due to the presence of 'Candidatus Phytoplasma' associated with "lethal yellowing" in palms and "yellows" in grapevine, and ' $\mathrm{Ca}$. Liberibacter' associated with "huanglongbing" in citrus. Their negative impact on agriculture may be worsened as a result of climate change. The management of these diseases is exploited in Africa (Ghana, South Africa and Mozambique), the Americas (Mexico, Chile, Guadalupe, Jamaica, and $\mathrm{Cuba}$ ), and Europe in selected areas where the prevalence of these diseases is severe giving rise to social and economic threats, affecting local agriculture and export of products. Knowledge and technologies available for detection and identification of these pathogens and new ones implemented by the project will be applied in the studied regions for epidemiological studies aimed at filling knowledge gaps. Integrated pest management strategies based also on innovative diagnostic and on exploited prevention tools such as reduced insecticide treatments, and improved resistant germplasm are applied and pest risk assessment schemes developed. The solutions that are under testing are evaluated to assess the economic and social impact of these diseases, and their distributive effects on the rural communities in the target countries The 22 partners from 12 countries are collaborating with local plant protection organizations, farmers and producers who are actively participating in training and practical application of the developed tools and schemes for the exploitation of the project outcome and results.

\section{Bacillus amyloliquefaciens subsp. plantarum strain D747: directly active and resistance inducer towards bacterial leaf spot of tomato}

\section{E. Biondi ${ }^{1}$, S.M. Perez ${ }^{1,2}$, M.R. Proto ${ }^{1}$, P. Pagliarani ${ }^{1}$, A. Bertaccini ${ }^{1}$ \\ ${ }^{1}$ Department of Agricultural and Food Sciences (DISTAL), Alma Mater Studiorum - University of Bologna, V. le Fanin, 44, Bologna, 40127, Italy; ${ }^{2}$ Instituto de Ciencias Agronómicas y Veterinarias, Universidad de O'Higgins, Rancagua, Avenida Bernardo O'Higgins, 611, 2820000, Rancagua, Chile.E-mail: enrico.biondi3@unibo.it}

The strain D747 of Bacillus amyloliquefaciens subsp. plantarum (Bam), the active substance of the bio-fungicide Amylo-X, was applied in vitro, to evaluate its ability in directly inhibiting the growth of Xanthomonas vesicatoria (Xv, strain IPV-BO 2684), the causal agent of bacterial leaf spot of tomato. Under climatic chamber conditions, Bam, as water suspensions of spores (SPS; $2 \mathrm{~g} / \mathrm{L}$ of Amylo-X) and 24 h-old living cells (LCS; $\mathrm{ca}$. $10^{7} \mathrm{CFU} /$ $\mathrm{mL}$ ), was applied at leaves and roots of tomato plants 'VF10'; after $48 \mathrm{~h}$, the plants were inoculated with the $X v$ strain. Sterile distilled water (SDW), streptomycin sulphate and acibenzolar-S-methyl were used as negative and positive controls, respectively. Moreover, the transcription abundance of selected genes related to the plant defence was investigated in response to LCS treatment at the root apparatus. The strain $\mathrm{D} 747$ was directly active in vitro by significantly inhibiting the $X v$ growth. Under high infection pressure, the tomato plants treated at leaves with Amylo-X (SPS) showed a significantly lower disease severity compared to SDW-treated plants, with a relative protection of $c a .53 \%$; also the tomato plants treated at the roots with LCS showed a significant reduction of the disease severity, providing a relative protection of $c a .45 \%$. The transcripts abundance of several genes (e.g. erf1, $p r 1, p r 4$ ), related to the host defence response, resulted significantly higher than that of control plants from 1 to $48 \mathrm{~h}$ after the root treatment, highlighting that Bam was able to induce a plant mediated response against $X v$ infection.

\section{Botrytis cinerea counteracts a grapevine chitinase by re- ducing its enzymatic activity and increasing the expression of the chitin synthase genes}

\author{
A. Bolzonello ${ }^{1}$, S. Tundo ${ }^{1}$, R. Marato $^{1}$, C. Castiglioni ${ }^{1}$, S. Odorizzi ${ }^{1}$, \\ S. Vincenzi ${ }^{2}$, L. Sella ${ }^{1}$, F. Favaron ${ }^{1}$ \\ ${ }^{1}$ Department of Land, Environment, Agriculture and Forestry, University \\ of Padova, Viale dell'Università 16, 35020, Legnaro, Italy; ${ }^{2}$ Department \\ of Agronomy, Food, Natural resources, Animals and Environment, \\ University of Padova, Viale dell'Università 16, 35020, Legnaro, Italy. \\ E-mail:angela.bolzonello@studenti.unipd.it
}

Chitin represents a main component of cell wall in filamentous fungi. In Botrytis cinerea the synthesis of this polymer involves a set of chitin synthases (Chs) grouped in seven classes. The relevance of Chs enzymes is demonstrated by reduction in growth and pathogenicity of $B$. cinerea $C h s$ disrupted mutants. During plant infection, chitin apposition to fungal cell wall is counteracted by host chitinases. Chitin synthesis and chitin degradation are, therefore, two opposite processes that can affect the infection outcome. We observed that the protease activity secreted by $B$. cinerea strain B0510 partially degrades a class IV chitinase of Vitis vinifera by cleaving the chitin binding domain (CBD) of this enzyme, which favors the attachment of chitinase to the fungal chitin substrate. In fact, the removal of the CBD reduces the chitinase activity by about $50 \%$. We hypothesized that the intact and the cleaved chitinase can differently affect $B$. cinerea chitin synthesis. To verify this aspect we have analyzed by quantitative PCR the expression of $C h s$ genes following B. cinerea treatment with intact and cleaved chitinase. We observed that the cleaved chitinase regulates positively the transcription of some Chs genes. These results show evidence that $B$. cinerea counteracts the host chitinase by reducing the activity of this enzyme and increasing the expression of chitin synthase genes.

Research work funded by Department of Land, Environment, Agriculture and Forestry, University of Padova (TESAF).

\section{A synthetic microbial community for the control of Fusarium wilt of banana}

\section{G. Bubici, M.I. Prigigallo}

Istituto per la Protezione Sostenibile delle Piante, Consiglio Nazionale delle Ricerche, via Amendola 165/A, 70126 Bari, Italy. E-mail: giovanninicola.bubici@cnr.it

Banana is the most produced fruit in the world. More than 150 million tons of bananas and plantains are produced annually for a business affair of 38.5 billion dollars. The entire banana industry has been threatening by Fusarium oxysporum f. sp. cubense (Foc) tropical race 4 (TR4), which is spreading alarmingly. We evaluated new biological control agents against Fusarium wilt of banana (FWB) to be used in mixture rather than as single strains. Strains of Pseudomonas spp., Bacillus spp., Streptomyces spp. and Trichoderma spp. were isolated from rhizosphere soils of banana crops in Tenerife island and selected for the in vitro antagonism against Foc. Effective strains were used to construct two synthetic 
microbial communities (SynCom1.0 and SynCom1.1) differing in the number of their member strains. Pot-experiments demonstrated that both SynComs can reduce FW of banana by $30 \%$ though the Foc population in the soil was not significantly affected, suggesting biocontrol mechanisms other than the direct antagonism against the pathogen. In vitro assays revealed that, besides the antagonism against $F o c$, most SynCom's member strains can antagonize each other, especially at the interspecies level, and that $F o c$ can even antagonize the beneficial microbes. However, based on these data, we were able to design a new SynCom (SynCom1.2) composed of three members that are both cross-compatible and resistant to Foc. SynCom1.2 was better than the previously designed consortia in controlling FWB, indicating that inconsistent biocontrol obtained with simple mixtures of microbes can be significantly improved by designing accurately tailor-made synthetic microbial communities.

This work was supported by the Horizon 2020 project entitled "Microbial Uptakes for Sustainable Management of Major Banana Pests and Diseases" (MUSA) (proposal number 727624; http://www.projectmusa.eu).

\section{Gliotoxin biosynthetic pathway distribution and organiza- tion among Trichoderma spp.}

\section{Bulgari ${ }^{1}$, L. Fiorini ${ }^{1}$, A. Gianoncelli ${ }^{2}$, E. Gobbi ${ }^{1}$}

${ }^{1}$ Piattaforma di Microbiologia Agroalimentare ed Ambientale (Pi.Mi.A.A.), AgroFood Lab, Department of Molecular and Translational Medicine, University of Brescia, viale Europa, 11, 25123, Brescia, Italy; ${ }^{2}$ Piattaforma di Proteomica, AgroFood Lab, Department of Molecular and Translational Medicine, University of Brescia, viale Europa, 11, 25123, Brescia, Italy.E-mail: emanuela.gobbi@unibs.it

Gliotoxin (GT) is a versatile secondary metabolite with a big paradox: it shows medical properties and plant protection abilities, but, at the same time, GT is a virulence determinant in the human pathogen Aspergillus fumigatus. Few studies have been carried out on GT production in Trichoderma spp. and its role in these species is still debated. In order to have a clear picture of the GT biosynthetic pathway (GT-BP) organization, six different Trichoderma spp. genomes were analyzed using A. fumigatus gli cluster as reference strain. These analyses revealed that the majority of the isolates had the GT-BP with the exception of T. gamsii. GT-BP in Trichoderma showed polymorphism related to different composition and architectures. In detail, two structures were identified, the first one in the GT-producing T. virens and the second one in all the other species here tested. The T. virens GT-BP was composed by core cluster ( 8 genes) separated from other essential genes located in three different loci. The second and more common structure in Trichoderma shared the organization of GT related genes in only one locus as in A. fumigatus, with the absence of $g l i H$ and with 2 putative gliF orthologues. Finally, gene expression analyses were set up in order to verify the correct identification of putative GT genes identified. Putative GT transcription factor ( $\mathrm{gliZ}$ ), previously identified in silico, was not expressed in both isolates.

\section{Exploit biodiversity in viticultural systems to reduce pest damage and pesticide use, and increase ecosystems ser- vices provision: the BIOVINE Project}

T. Caffi ${ }^{1}$, J. Armengol Forti ${ }^{2}$, P. Kehrli ${ }^{3}$, A.-M. Ranca ${ }^{4}$, S. Sirca ${ }^{5}$, A. Wipf $^{6}$, V. Rossi ${ }^{1}$

${ }^{1}$ Department of Sustainable Crop Production, Università Cattolica del Sacro Cuore, via Emilia Parmense 84, 29122 Piacenza, Italy; ${ }^{2}$ Instituto Agroforestal Mediterráneo, Universitat Politècnica de València, Camino de Vera S/N, 46022 Valencia, Spain; ${ }^{3}$ Département fédéral de l'économie, de la formation et de la recherche, Agroscope, Domaine stratégique de recherche Protection des végétaux, Route de Duillier 50, CP 1012, 1260 Nyon1, Suisse; ${ }^{4}$ Research Station for Viticulture and Enology Murfatlar, Calea Bucuresti, nr. 2, Murfatlar, Romania; ${ }^{5}$ Plant Protection Department, Agricultural Institute of Slovenia, Hacquetova ulica 17, 1000 Ljubljana, Slovenia; ' Agroécologie, AgroSup Dijon, CNRS, INRA, Univ. Bourgogne Franche-Comté, 21000 Dijon, France. E-mail: vittorio.rossi@unicatt.it

Organic vineyards still rely on large external inputs to control pests. The BIOVINE project aims to develop natural solutions based on plant diversity to control pests and reduce pesticide dependence. The capability of plants of increasing the ecosystem resistance to pests and invasive species is a well-known ecosystem service. However, monocultures (including vineyards) do not exploit the potential of plant diversity. BIOVINE aims to develop new viticultural systems based on increased plant diversity within (e.g., cover crops) and/or around (e.g., hedges, vegetation spots, edgings) vineyards by planting selected plant species for the control of arthropods, soil-borne pests (oomycetes, fungi, nematodes), and foliar pathogens. Different experiments, both at plot and on farm level, have been settled up with candidate plants as cover crops by each Partner. The first year of trials and assessments was carried out in order to evaluate the ability of such species to: i) attract or repel target arthropod pests; ii) conserve/promote beneficials; iii) control soil-borne pests by mean of biofumigation; iv) carry mycorrhizal fungi to vine root system to increase plant health (growth and resistance); and v) control foliar pathogens by reducing the inoculum spread from soil. An ex-ante assessment of the innovative viticultural systems sustainability was carried and the systems are under evaluation in France, Italy, Romania, Slovenia, Spain and Switzerland for a 2 -year period. Innovative viticultural systems should represent an improved way for pest control in organic viticulture, meanwhile they should positively affect functional biodiversity and ecosystem services. New control strategies may provide financial opportunities to vine growers and lower the use of pesticides.

The authors acknowledge the financial support for this project provided by transnational funding bodies, being partners of the H2020 ERA-net project, CORE Organic Cofund, and the cofund from the European Commission.

\section{Innovative studies for assessing and increasing soil ecosys- tem services in viticulture}

\section{T. Caffi, F. Fedele, S. Poni, V. Rossi}

DIPROVES - Sustainable Crop and Food Protection, Università Cattolica del sacro Cuore, Via E. Parmense 84, 29122 Piacenza, Italy. E-mail: tito.caffi@unicatt.it

Soil is a key components of viticultural systems. Soil health has been defined as the continued capacity of soil to function as a vital living ecosystem that sustains plants, animals, and humans. Since soil provides several ecosystem services, including natural control of pests, it is essential to understand conditions and management actions enhancing natural biocontrol. Two research projects are ongoing to evaluate the effect of soil management on soil health and related ecosystem services. The LIFE+ project SOIL4WINE "Innovative approach to soil management in viticultural landscapes" aims to develop new viticultural systems focused on achieving better soil 
management through providing cost-effective solutions improving soil ecosystem services. The proposed solutions focus, for instance, on: i) cover crops in the mid-row and in headlands around vineyard to prevent erosion, increase biodiversity and pest control; ii) permanent organic mulching in the vine row for sustainable weed management, iii) superficial water control or underground drainage system to regulate excess of water; iv) change of soil management equipment to reduce compaction and remove hardpan. The ECORESILIENTE project uses the experimental set up of SOIL4WINE for better investigating soil biodiversity by studying the microbiome of the vineyard soil by using a functional approach on both pathogen and beneficial microorganisms, and fermentative species. These projects define guidelines and good practices for soil and soil coverage management able to increase and protect soil health and biodiversity, and for exploiting the natural control of vineyard pests (oomycetes, fungi, arthropods, mites and nematodes).

The authors acknowledge the financial support provided by the European Commission for the Soil4Wine project and the Università Cattolica del Sacro Cuore for the project ECORESILIENTE.

\section{Fast dissection of virome infecting different hosts by NGS approaches}

\section{Calassanzio, J. Zhang, A. Prodi, C. Ratti}

Department of Agricultural and Food Sciences, University of Bologna, Viale G. Fanin, 40127, Bologna, Italy.E-mail: claudio.ratti@unibo.it

Next generation sequencing (NGS) is no longer an alternative for simultaneous detection of known and emergent viruses present in different hosts. The development of new approaches capable of dissecting the virome of filamentous fungi and plant samples was performed. Therefore, the heterogeneity of the genome and the variety of effects that viruses can cause in the host, make them a perfect model for the development of NGS techniques. The semiconductor-based sequencer Ion Torrent $^{\mathrm{TM}}$ and the nanopore-based sequencer MinION have been exploited to analyze DNA and RNA referable to viral genomes. DNA libraries were generated from dsRNA and VANA and analyzed by the IonTorrent and the MinION PCR sequencing kit. Then 5' and 3' ends of viral RNA genomes including untranslated regions (UTRs) were determined using a RACE protocol based. Moreover, MinION Direct RNA Sequencing was performed with the aim of bypassing the PCR steps and the 3' and 5' RACE. Comparison with GenBank accessions and sequence analysis allowed to identify more than 40 putative viral species belonging to different genera or to unclassified mycoviruses. Some of these mycovirus genera have been studied as inducers of hypovirulence in several phytopathogenic fungi, therefore future works will focus on the comparison of the morphology and physiology of the fungal strain infected and cured by the viruses identified and their possible use as a biocontrol agent. On the other hands MinION could be developed as a reliable tool for plant material certification or for the identification of new viral agents.

\section{Antibacterial activity of hydrolysable tannins against Pseudomonas syringae pv. tomato and their potential biostimulant activities on tomato plants}

\section{P. Canzoniere, S. Francesconi, G.M. Balestra}

Dipartimento di Scienze Agrarie e Forestali (DAFNE), Università della Tuscia, Via San Camillo de Lellis, snc, 01100, Viterbo, Italy. E-mail: balestra@unitus.it
Pseudomonas syringae pv. tomato (Pst), the phytobacteria causal agent of tomato speck disease, represents a worldwide relevant cause of economic losses for tomato crops. Its control is mainly related to balanced agronomic practices and preventive use of commercial formulations based on cupric salts. The EU restrictions about the copper use and the needs to reduce the chemicals in agriculture require to find sustainable alternative that can replace/reduce cupric salts usually employed on bacterial diseases control. In this study, the in vitro and in vivo tannins antibacterial activity was verified respect to Pst. Furthermore, tannins biostimulant activity was verified on tomato plants. Regarding antibacterial activity, incorporated tests were carried out in vitro at different tannin concentrations $(0.5-5 \% \mathrm{w} / \mathrm{v})$. In vivo tests were conducted at two different plant stages $\left(1^{\text {st }}\right.$ and $4^{\text {th }}$ true leaf stage development) to verify both antibacterial and biostimulant activities applying tannins by foliar-spray treatment at $1 \% \mathrm{w} / \mathrm{v}$ before bacterial inoculation. Biostimulant activity on plants was verified by the biomass weight and the nitrogen content index, and antibacterial activity was verified by Pst epiphytic survival. Results highlights that two of the tested tannins (UTUST-1, 3) expressed a remarkable biostimulant and antibacterial activity both individually and in association with reduced doses $(1 / 2$ F.D.) of copper hydroxide. The properties of tannins utilized and their potentiality in organic plant protection strategies to control phytobacteria have been discussed.

\section{Phylogenetic and pathogenic analyses of different Fusarium proliferatum strains isolated from different plant matrices}

\section{E. Cappelletti ${ }^{1}$, M.T. Senatore ${ }^{1}$, I. Alberti ${ }^{2}$, A. Prodi ${ }^{1}$}

${ }^{1}$ Department of Agricultural Sciences, Alma Mater Studiorum University of Bologna, Viale G. Fanin, 44, 40127, Bologna, Italy; ${ }^{2}$ Council for Agricultural Research and Economics-Research Center for Cereal and Industrial Crops, Viale Giovanni Amendola 82, 45100 Rovigo, Italy. Email:eleonora.cappellett5@unibo.it

Fusarium proliferatum (Matsushima) Nirenberg is a polyphagous and widespread pathogenic species. Its pathogenic capacity is proved on several different agricultural plants, in addition to its ability to produce high levels of a wide variety of mycotoxins, such as fumonisins, which are a hazard for both human and animal health. Taking into account these issues, different $F$. proliferatum strains were analyzed both from the molecular point of view and virulence on host plants. Forty-five strains of $F$. proliferatum were subjected to a phylogenetic analysis based on partial sequencing of $T E F-1 \alpha$ and $F U M 1$ genes. In parallel, fourteen strains of $F$. proliferatum, isolated from five different host plants, were tested for their pathogenicity. Cross-pathogenicity tests were performed in the greenhouse on six different plant species susceptible to the pathogen. Asparagus, onion, Welsh onion, garlic, maize, and durum wheat plant species were artificially inoculated with a $F$. proliferatum conidia suspension and compared with the control. Both TEF-1 $\alpha$ and FUM1 phylogenetic trees showed two distinct main groups of $F$. proliferatum strains. $T E F-1 \alpha$ tree highlighted a higher variability among the isolates respect to the FUM1 one, while no direct correlation with the strain host species was observed. Furthermore, it was possible to confirm the presence of the fumonisin biosynthesis gene (FUMI) for all the isolates. In pathogenicity tests, even if some strains have shown greater virulence than others, there does not appear to be a correlation between the level of virulence of a given strain and its host origin. 
Persistence of Heterobasidion annosum s.s. and $H$. irregulare in artificially inoculated stone pine (Pinus pinea) $\operatorname{logs}$

\section{P. Capretti, C. Aglietti, L. Giusti, L. Ghelardini}

Department of Agriculture, Food, Environment and Forestry (DAGRI) University of Florence, Piazzale delle Cascine 28 - 50144 Firenze. Email:paolo.capretti@unifi.it

The stone pine (Pinus pinea L.) is a species of ecological and economical importance, and an iconic landscape tree in the Mediterranean areas. Along the Tyrrenian coast near Rome (Italy, Latium), for about $100 \mathrm{~km}$ between the Circeo Park and Fregene, $P$. pinea forests suffer heavy damage by Heterobasidion root rot. The disease is especially virulent in Castel Porziano and Castel Fusano, where both $H$. annosum s.s. and $H$. irregulare, a quarantine species of North American origin, are found. The area colonized by the pathogens increases slowly by natural dispersion, but fast spread might occur through movement of infected plant material. To investigate the saprophytic behavior of the two fungi, and their ability to survive and sporulate on cut wood, logs (about $25 \times 50 \mathrm{~cm}$ ) were cut from stone pine trunks in a disease-free area, transported to the lab, and inoculated with 4 beech wood dowels colonized by one out of six strains of $H$. annosum s.s. or six strains of $H$. irregulare. Inoculated logs were wrapped in plastic bags and kept indoor at $15-27{ }^{\circ} \mathrm{C}$ in the dark for 5 years. The number of carpophores and the degree of wood decay of each $\log$ were assessed at the end of the experiment. H. irregulare strains, although less effective in decaying wood, produced more abundant and larger carpophores for the whole study period. Results confirm that the risk of spreading the invasive pathogen $H$. irregulare through cut wood may remain extremely high for a very long time.

\section{Morphological and genetic characterization of Lirula nervisequia isolated from silver fir (Abies alba) needles}

\section{Cerboneschi, P. Capretti, S. Tegli}

Department of Agriculture, Food, Environment and Forestry (DAGRI), Plant Pathology and Entomology section, University of Florence ITALY.E-mail: matteo.cerboneschi@unifi.it

Silver fir (Abies alba) is a large coniferous tree mainly distributed in montane areas in Central Europe but it is also present in southern and eastern Europe. Although it has an ancient origin, silver fir sometimes shows crisis situations due to environmental challenges and parasitic attacks caused mainly by fungi and insects. During the last decades the European fir trees, and particularly the Italian ones, have shown repeated cycles of crisis, sometimes sudden deaths by Armillaria sp. but also eradication by Heterobasidion abietinum of apparently healthy trees. Damage caused on the crown by Lirula nervisequia (DC ex Fr.) Darker on young silver fir trees has been observed in Vallombrosa Forest since the ' $50 \mathrm{~s}$. This foliar pathogen causes discoloration and dead of foliage, never followed by abscission, on two or more year old needles. The fungus is presumed to be endemic in Vallombrosa Forest, and its epidemic spread occurs only when the weather conditions as well as the whole biological ecosystem are favorable. Humid spring followed by summer drought is considered one of the main causes of sudden symptomatic manifestations. However, in this condition the dissemination of L. nervisequia is limited, and thus the production and spread of ascospores is concentrated during spring and autumn. Modern microscopic investigations, based on deconvolution techniques, have produced a more detailed morphological characterization of $L$. nervisequia in several different phases of its biological cycle, and a more in-depth study was carried out for the genetic characterization of ITS region of the species.

Identification of antifungal metabolites from a Streptomyces sp. effective against Fusarium graminearum and $\boldsymbol{F}$. culmorum

\section{E.M. Colombo ${ }^{1}$, S. Batey ${ }^{2}$, A. Kunova ${ }^{1}$, B. Wilkinson ${ }^{2}$}

${ }^{I}$ DeFENS - Department of Food, Environmental and Nutritional Sciences, Università degli Studi di Milano, via Celoria 2, 20133, Milano, Italy; ${ }^{2}$ Department of Molecular Microbiology, John Innes Centre, Norwich Research Park, Norwich NR4 7UH, UK. E-mail: elenamaria.colombo@unimi.it

Streptomycetes are gram-positive bacteria belonging to the phylum Actinobacteria, and the source of two thirds of all antibiotics in current clinical use. They are ubiquitous in soil, but can establish tight interactions with plants, producing a wide range of metabolites limiting pathogen development and promoting plant growth. Severe restrictions of fungicide registration and use in agriculture call for new and original approaches to manage plant diseases and mycotoxin contamination of food and feed, for example the use of biocontrol agents. During the research focused on the selection of Streptomyces spp. active against fusarium head blight and crown rot on wheat, we identified a promising strain able to limit mycelial development and deoxynivalenol (DON) production in infected grains. The strain DEF48 showed $45 \%$ and $65 \%$ inhibitory activity against $F$. graminearum and $F$. culmorum respectively using the dual-culture plate method, and limited up to $99 \%$ the DON amount in grains under microsilage conditions. Therefore, its antifungal secondary metabolites produced in vitro were characterized. Bioassay guided isolation was used to identify the metabolites by evaluating the activity of fractions obtained from agar plate extraction against the target pathogen. In addition, Illumina sequencing of its genome was performed. The integration of the antiSMASH bioinformatic analysis to identify secondary metabolite biosynthetic gene clusters, literature searches for antifungal compound composition, and the screening of the UV spectra of the active fractions allowed us to identify the candidate antifungal molecule. It represents a promising candidate to be employed in biological control of mycotoxigenic fusaria in cereals.

\section{NoPv1, a new antifungal peptide able to protect grapevine} from Plasmopara viticola infection

M. Colombo ${ }^{1}$, S. Masiero ${ }^{2}$, M. Perazzolli ${ }^{1}$, S. Pellegrino ${ }^{3}$, R. Velasco ${ }^{4}$, R. Musetti ${ }^{5}$, S.L. Toffolatti ${ }^{6}$, S. Vezzulli ${ }^{1}$, P. Pesaresi ${ }^{2}$

${ }^{1}$ Research and Innovation Centre, Fondazione Edmund Mach, via E. Mach 1, 38010 San Michele all'Adige, Italy; ${ }^{2}$ Department of Biosciences, University of Milano, Via Celoria 26, 20133 Milano, Italy; ${ }^{3}$ Department of Pharmaceutical Sciences, University of Milano, Via Venezian 21, 20133 Milano, Italy; ${ }^{4}$ CREA, Research Centre for Viticulture and Enology, Via XXVIII Aprile 26, 31015 Conegliano, Italy; ${ }^{5}$ Department of Agricultural, Food, Environmental and Animal Sciences, University of Udine, via delle Scienze, 206, I-33100 Udine, Italy; ${ }^{6}$ Department of Agricultural and Environmental Sciences Production, Landscape, Agroenergy, University of Milano, via Celoria 2, 20133 Milano, Italy.E-mail: monica.colombo@fmach.it 
With the human population expected to reach 9.7 billion by the middle of this century, increasing agricultural productivity through the prevention of crop losses to pests and diseases has assumed considerable urgency. Modern agriculture strongly relies on pesticides to avoid losses and produce high-quality foods. Fungicides in particular represent the majority of plant protection products, with viticulture representing one of the first sector in terms of fungicide usage which has a serious negative impact on the environment and on human/animal health. As a consequence, crop protection is now oriented towards a rational use of pesticides and the development of eco-friendly pesticide alternatives. Among the available biotechnological solutions, peptide aptamers, i.e. artificial, high-affinity short peptides that specifically inhibit a target molecule through protein interference, are emerging as novel molecular tools.

The oomycete Plasmopara viticola Berl. \& De Toni is the causal agent of grapevine downy mildew, a worldwide destructive disease of primary importance for viticulture. Within the frame of GraptaResistance research project (https://sites.unimi.it/ graptaresistance/), funded by Fondazione Cariplo, we identified a novel peptide aptamer, named $\mathrm{No} P v 1$ (No P. viticola 1 ), able to counteract $P$. viticola infection ex vivo (leaf disks) and in vivo (potted plants). NoPv1 has been isolated from a peptide library, through the yeast two-hybrid strategy, using the $\mathrm{PvCesA} 2$ protein ( $P$. viticola cellulose synthase 2) as a bait. In addition, we demonstrated that NoPv1 targets specifically $P$. viticola as well as Phytophtora infestans, without affecting off-target organisms and being toxic for human cells.

\section{Spatial pattern of esca disease in commercial vineyards in Marche region}

\section{J. Concas, S. Murolo, V. Mancini, R. Foglia, G. Romanazzi}

Department of Agricultural, Food and Environmental Sciences, Marche Polytechnic University, Via Brecce Bianche, 60131 Ancona, Italy. E-mail: g.romanazzi@univpm.it

Esca is one of the most important diseases of grapevine (Vitis vinifera), and it is a limiting factor for the production in most viticultural areas. The study was carried out in three commercial vineyards (OS1, OS2, CAS), located in Marche region. The position of each symptomatic plant was reported on a map and the disease severity was recorded using an empirical scale of with five degrees, where: $0=$ symptomless/healthy plant; $1=$ plant showing 1-10 leaves with symptoms; 2 = plant showing up to $50 \%$ of symptomatic leaves; 3 = plant with more than $50 \%$ of canopy with symptoms; 4 = apoplectic stroke. During the surveys, a consistent percentage of plants showing "leaf tiger-stripes" (ranging from $10 \%$ to $50 \%$ ) and apoplectic stroke (ranging from $5 \%$ to $20 \%$ ) was recorded. The data collected during the surveys were statistically elaborated by SYSTAT v.13 software, which generated maps of esca disease spread and severity. The presence of inter-row and intra-row disease gradient was determined by a regression curve, which represents the percentage of symptomatic plants in relationship with the distance from the edge of the vineyard. The data processing allowed to verify in 2017 and 2018 that i) none of the vineyards showed a gradient of esca disease among the rows; ii) there was a West-East gradient of disease in the vineyard OS1, and in the vineyard OS2 a greater concentration of disease in the center than the edges was observed. The obtained result represents a further set of information that can allow a better understanding of the epidemiology of esca disease.
BNYVV collective infectious units: RNA-RNA interactions driving the integrity preservation of a multicomponent genome

\section{Dall'Ara ${ }^{1}$, D. Poli ${ }^{1}$, M. Patrian ${ }^{1}$, C. Ratti ${ }^{1}$, F. Michel ${ }^{2}$, E. Klein ${ }^{2}$,} S.E. Bouzoubaa ${ }^{2}$, D. Gilmer ${ }^{2}$

Dipartimento di Scienze e Tecnologie Agro-Alimentari, Area Patologia Vegetale, Università di Bologna, Bologna, Italy; ${ }^{2}$ Institut de Biologie Moléculaire des Plantes, Integrative Virology, CNRS UPR2367, Université de Strasbourg, Strasbourg, France.E-mail: mattia.dallara5@unibo.it

Multipartite viruses possess a genome split in two or more components packaged in independent particles. If on one hand benefits of a multipartite life style are still under debate, on the other hand virologists agree on the fact that the condition to assure the complete set of genomic segments in each infected cell implies a high level of multiplicity of infection increasing with the number of genome components and resulting unsustainable in terms of viral replication. Beet necrotic yellow vein virus (BNYVV) is a multipartite RNA virus with the highest number of genomic segments (up to 5) within the Baltimore's class IV that concerns ssRNA(+) viruses. BNYVV set point genomic formula is variable depending by the infected host, moreover short genomic RNAs are more abundant than longer ones. Demonstrating that all genomic segments are present within the single infected cell it appears likely that a specific mechanism guarantees the coordinated movement of each segment as collective infective units. To minimize the cost of the genome integrity preservation, it is proposed a network of RNA/RNA interactions that determines the recognition and the mobilization of at least one of each genomic RNA in a modular RNP complex. Such complex must be considered as the mobile infectious unit during viral spread in the plant. In the model where vRNAs interact each other to form a mobile infective unit, RNA1 and RNA2 interaction domain has been identified. The rationale of such interaction has been provided using BNYVV and beet soil-borne mosaic virus chimeras.

\section{Empty cowpea mosaic virus-based particles as potential nanotools for plant protection against diseases}

\section{Danzi, R. Zampieri, A. Polverari, L. Avesani, E. Vandelle}

Department of Biotechnolgy, University of Verona, Strada le grazie 15, 37134 Verona, Italy. E-mail: elodiegenevieve.vandelle@univr.it

Pursuing plant protection through an eco-friendly and sustainable approach can be accomplished by exploiting naturally-occurring peptides, carrying an antimicrobial activity (antimicrobial peptides, AMPs) or alternatively able to stimulate the plant immune system (immunity triggering peptides, ITPs). However, one main limit in the use of peptides for plant protection is their poor stability and the cost of their production. An answer to such issue could be found in the nanotechnologies. In particular, in the era of nanotechnology, viruses as nanotools gained a lot of attention from many research fields because of their peculiar properties like their regular structure and predictable conformation, besides their ability to tolerate extreme chemical and physical conditions. Moreover, they can be produced as empty virus-like particles (eVLPs), i.e. devoid of any genetic material, using a cost-effective production system based on molecular farming. Thanks to these properties, cowpea mosaic virus (CPMV) is one of the most studied plant viruses exploited for the exposition of molecules ranging from small organic compounds to active enzymes. In this context, the aim of our current project is to develop eCPMV particles harbouring AMP or ITP to fight against microorganisms or induce 
plant defence responses, respectively. Whereas the production of eCPMVAMP is requiring further optimization to cope with AMP features, which raise problems in terms of particle assembly and/or stability, we obtained eCPMV-ITP particles with a good yield and integrity. Moreover, the treatment of Arabidopsis thaliana plantlets with eCPMV-ITP showed the ability of the functionalized nanoparticles to induce the plant immune system.

\section{Application of soil metabolomics to analyze vineyards (in Veneto) affected by fanleaf degeneration}

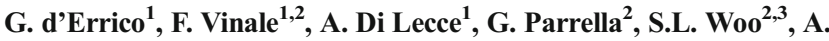

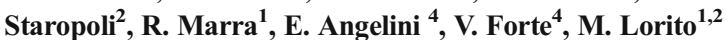

${ }^{1}$ University of Naples Federico II, Department of Agricultural Sciences, Via Università 133, 80055 Portici (NA), Italy; ${ }^{2}$ National Research Council, Institute for Sustainable Plant Protection, Via Università 133, 80055 Portici (NA), Italy; ${ }^{3}$ University of Naples Federico II, Department of Pharmacy, Via D. Montesano 49, 80131 Naples, Italy; ${ }^{4}$ CREA Viticulture and Enology, Viale XXVIII aprile 26, 31015 Conegliano (TV), Italy.E-mail: giada.derrico@unina.it

The dagger nematodes Xiphinema index are vectors of grapevine fanleaf virus (GFLV), responsible for the fanleaf degeneration in vineyards, mainly occurring when grapevines are replanted in old vineyards that can be largely infested with plant-parasitic nematodes. The aim of this work was to use metabolomic analysis to characterize the soil biochemical profiles in vineyards affected by fanleaf degeneration and infested by plant-parasitic nematodes, in order to study the association between these biotic stress factors. In this context, soil metabolomics could be a useful tool to investigate the entire metabolite complex of the soil ecosystem, providing useful information about the interactions in the soil community that influence physiological, pathological, and symbiotic processes. A survey in the most traditional viticulture areas of Veneto was performed by collecting more than two-hundred soil samples, during different plant development stages, to analyze and determine the virus and nematode vector distribution. Numerous samples resulted infested by $X$. index, as well as by other dangerous plant-parasitic nematodes. Water/methanol extracts were obtained from soils, and subjected to both targeted and untargeted metabolomic analyses by liquid chromatography-mass spectrometry (LC-MS qTOF). Principal component and LC-MS predictive modelling analyses revealed distinct biochemical profiles of collected soils, associated to grapevine phenological stages. Knowledge of specific metabolites identified, as well as the effects on the pathogenic biota, will help to increase the understanding of vineyard ecology, and aid in the diagnostic of virus diseases and their vectors.

Work supported by DI.VI.NE project (PSR Regione Veneto 2018-22).

\section{Spatial distribution of the dagger nematode Xiphinema index and its associated grapevine fanleaf virus (GFLV)} across vineyard ecosystems in Veneto

G. d'Errico ${ }^{1}$, F. Vinale ${ }^{1,2}$, S.L.Woo ${ }^{2,3}$, R. Marra ${ }^{1}$, E. Angelini ${ }^{4}$, V. Forte $^{4}$, M. Panzeri ${ }^{4}$, L. Aiello ${ }^{4}$, N. Bertazzon ${ }^{4}$, S. Casarin ${ }^{4}$, F.P. d'Errico' $^{1}$, M. Lorito ${ }^{1,2}$

${ }^{1}$ University of Naples Federico II, Department of Agricultural Sciences, Via Università 133, 80055 Portici (NA), Italy; ${ }^{2}$ National Research Council, Institute for Sustainable Plant Protection, Via Università 133, 80055 Portici (NA), Italy; ${ }^{3}$ University of Naples Federico II, Department of Pharmacy, Via D. Montesano 49, 80131 Naples, Italy, ${ }^{4}$ CREA Viticulture and Enology, Viale XXVIII aprile 26, 31015 Conegliano (TV), Italy. Email: giada.derrico@unina.it
The nepovirus grapevine fanleaf virus (GFLV) is specifically transmitted by the nematode Xiphinema index. GFLV is responsible for the infectious degeneration in grapevines causing serious losses in yield and reducing the longevity of the vines. Additionally, $X$. index provokes plant stunting, chlorosis, root swellings or galls and root necrosis. Increased knowledge of the spatial distribution of the vector, both horizontal and vertical, and of the associated GFLV infections, is crucial to efficiently control the disease. Twenty-eight vineyards in Veneto region were surveyed and sampled both for soil and symptomatic leaves. $X$. index occurred in $47 \%$ of soil samples exhibiting a different abundance among samples (from 1 to 282 individuals per $500 \mathrm{ml} / \mathrm{soil}$ ). In particular, vineyards in Treviso province were less infested than those in Verona province. GFLV was detected in about $65 \%$ of the plant samples, ranging from 10 to $99 \%$ depending on the vineyard. In symptomatic vineyards, vertical distribution data showed that the highest number of $X$. index occurred at 40 to $60 \mathrm{~cm}$ depth, corresponding to layers with the highest root densities. Horizontal distribution revealed a significant aggregative pattern, but no significant neighborhood structure of nematode densities. Nematode patches correlated significantly with those of GFLV-infected grapevines. Nematode and virus distribution was shown to extend preferentially parallel to vine rows, probably due to tillage practices. Results are discussed in relation to the needs for surveillance and management strategies of fanleaf degeneration in the most traditional wine production areas.

Work supported by DI.VI.NE project (PSR Regione Veneto 2018-22).

\section{Biocontrol activity of Aureobasidium pullulans against the soil pathogen Rhizoctonia solani}

\section{A. Di Francesco ${ }^{1,2}$, M. Corbetta ${ }^{2}$, D. Baldo ${ }^{2}$, C. Ratti $^{2}$, E. Baraldi ${ }^{1,2}$ \\ ${ }^{1}$ CRIOF-Department of Agricultural and Food Sciences, University of Bologna, Via Gandolfi 19, 40127 Cadriano, Italy; ${ }^{2}$ Department of Agricultural and Food Sciences, University of Bologna, Viale Fanin, 46, 40127 Bologna, Italy.E-mail:alessand.difrancesc3@unibo.it}

The most common bean plant diseases are caused by soil-borne pathogens displaying important economic losses in the world. Strains L1 and L8 belonging to Aureobasidium pullulans species were tested in vitro as biocontrol agents (BCAs) against Rhizoctonia solani (AG-4). The non-volatile metabolites produced by L1 and L8 strains inhibited the pathogen mycelial growth of $87.9 \%$ on average, not showing significant differences between the two strains. The lowest pathogen diametric growth inhibition was displayed by both yeast volatile metabolites that reduced the colony growth of $R$. solani significantly and in a same way compared to the control, with an average of $10.5 \%$. By in vivo assay, L1 and L8 strains showed the ability to control the pathogen virulence probably through the biofilm formation around the bean plant roots, as confirmed by SEM analysis and spectrophotometer assay. Moreover, studies investigated the efficacy of L1 and L8 strains to stimulate the bean plant roots and leaves growth, acting in this way not only as BCAs but also with a biostimulator function.

\section{Aureobasidium pullulans $\mathrm{L} 1$ and $\mathrm{L8}$ strains as an alterna-} tive control strategy of Neofusicoccum parvum

\author{
A. Di Francesco ${ }^{1,4}$, C. Rusin ${ }^{2}$, M. Di Foggia ${ }^{3}$, A. Rombolà ${ }^{4}$, E. \\ Baraldi $^{1,4}$
}

${ }^{1}$ CRIOF-Department of Agricultural and Food Sciences, University of Bologna, Via Gandolfi, 19, 40127 Cadriano, Italy; ${ }^{2}$ Universidade Estadual do Centro Oeste, Guarapuava, Brazil; ${ }^{3}$ Department of 
Biomedical and Neuromotor Sciences, University of Bologna, Via Belmeloro, 8/2, 40126 Bologna, Italy; ${ }^{4}$ Department of Agricultural and Food Sciences, University of Bologna, Viale Fanin, 46, 40127 Bologna, Italy.E-mail:alessand.difrancesc3@unibo.it

Aureobasidium pullulans L1 and L8 strains displayed a great ability to reduce Neofusicoccum parvum conidia germination of almost $50 \%$ and totally stopped its sporification in wood of 'Gala' and 'Fuji' apple cultivars. By in vitro tests, the antagonistic activity displayed by non-volatile metabolites produced by both yeasts was quantified as a $70 \%$ of reduction of the pathogen mycelial growth. On apple fruit, the pathogen severity was reduced by $66 \%$ and $50 \%$ in 'Gala', and $70 \%$ and $60 \%$ in 'Fuji' by L1 and L8 respectively. In wood, no statistical differences were observed between L1, L8 and the control, except for 'Fuji' wood, where L8 strain displayed a wood lesion reduction of $23 \%$ with respect to the untreated control. Infrared analysis showed how the presence of both strains on woody tissues helped preserving cellulose content, preventing in this way the faster wood degradation by the pathogen. In addition, L1 and L8 simply colonized the wood surface, probably displaying an endophytic attitude, an essential requirement for controlling $N$. parvum in field.

\section{From male-killers to plant pathogens? Investigating the capacity of insect-associated Arsenophonus symbionts to colonize plants}

\section{J. Dittmer ${ }^{1}$, N. Arricau-Bouvery ${ }^{2}$, B. Batailler ${ }^{2}$, P. Nadal-Jimenez ${ }^{3}$, G. Hurst ${ }^{3}$, X. Foissac ${ }^{2}$, F. Faoro ${ }^{1}$}

${ }^{I}$ Dipartimento di Scienze agrarie e ambientali (DISAA), Università degli Studi di Milano, Via Celoria 2, 20133 Milano, Italy; ${ }^{2}$ INRA BordeauxAquitaine, UMR 1332 Biologie du Fruit et Pathologie, 71 avenue Edouard Bourlaux, 33882 Villenave d'Ornon, France; ${ }^{3}$ Institute of Integrative Biology, University of Liverpool, Crown Street, Liverpool L697ZB, UK.E-mail: jessica.dittmer@unimi.it

Bacteria of the genus Arsenophonus represent one of the most widespread clades of insect endosymbionts and establish diverse symbiotic interactions with their insect hosts, ranging from the initially discovered male-killing strain of parasitoid wasps to obligate mutualists of several blood-feeding insects. Within this group of insect symbionts, two strains have been identified as insect-vectored plant pathogens, causing diseases in strawberry and sugar beet. In both cases, the bacteria are vectored by planthoppers and accumulate in the plant phloem, ultimately causing yellows and plant death. Hence, the switch from an ancestral purely insect-associated to a multi-host lifestyle occurred independently multiple times within this genus. These symbionts therefore represent outstanding model systems to investigate bacterial adaptations at the early stages of transition towards insect-vectored phytopathogens. Here, we investigate whether the ability to infect plants was achieved via genomic adaptations compared to other Arsenophonus strains or whether the genetic repertoire within this clade is sufficiently versatile to allow rapid adaptations to plant host environments, should the ecological opportunity arise. To this end, we performed experimental transmission experiments with a GFP-tagged strain of the male-killer A. nasoniae isolated in pure culture in combination with comparative genomics. Specifically, we searched the available Arsenophonus genomes for orthologs of known effector molecules mediating plant pathogenicity and discuss whether numerous insects carrying Arsenophonus might be reservoirs of potential plant pathogens.
This work was supported by the European Union's Horizon 2020 research and innovation programme under the Marie Sklodowska-Curie grant agreement No 792813

\section{Effect of hydrosols against pathogenic telluric fungi of melon and insect vectors of cucurbit viruses}

\section{Donati, S. Bertin, L. Trincone, A. Haegi, A. Gentili, L. Ferretti}

Consiglio per la ricerca in agricoltura e l'analisi dell'economia agraria (CREA) - Centro di ricerca Difesa e Certificazione (CREA DC) sede di Roma, Via C. G. Bertero 22 - 00156 Roma (Italy). E-mail: luca.ferretti@crea.gov.it

In recent years, several regulations and safety measures were issued to limit the use of pesticides and reduce their impact on both human health and environment. Nowadays the development of sustainable control strategies is becoming the main challenge in agriculture. In this context, within the project CUCURBIOMID several natural compounds are under evaluation for their efficacy against pathogens and key-insects of two high-income crops of Latium region, zucchini squash (Cucurbita pepo L.) and melon (Cucumis melo L.). Among these compounds, thyme and rosemary hydrosols (low-cost by-products of essential oils distillation), were tested for their effect against pathogenic telluric fungi of melon and insect vectors of cucurbit viruses (e.g. aphids and whiteflies). The minimum inhibitory concentration (MIC) of the hydrosols were evaluated against Fusarium oxysporum f. sp. melonis (FOM), causal agents of vascular wilt disease (trachemycosis). Both hydrosols showed fungistatic and fungitoxic activity at $20 \%$ and $40 \%(\mathrm{v} / \mathrm{v})$, respectively. Thyme and rosemary hydrosols were also tested for both their toxic and repellent effects against Aphis gossypii, a vector of potyviruses infecting zucchini squashes. The preliminary results obtained in laboratory bioassays showed a negative impact of these compounds on both the survival and settling behavior of the aphid compared to the water control. These early laboratory results encourage further assays, towards a possible application of these and other hydrosols in protected and field conditions.

This work is supported by Latium Region, Convenzione N. Registro 21706, CUCURBIOMID project (ID 85-2017-15024) within the Call: "Progetti di Gruppi di Ricerca - Conoscenza e cooperazione per un nuovo modello di sviluppo" (L.R. 13/2008 - art. 4).

\section{A new approach to identify Xylella fastidiosa in plant}

L. Faino ${ }^{1}$, V. Scala ${ }^{2}$, A. Albanese ${ }^{1}$, N. Pucci $^{2}$, A. Grottoli ${ }^{1,3}$, V. Modesti $^{2}$, A. L'Aurora ${ }^{2}$, M. Reverberi ${ }^{1,3}$, S. Loreti $^{2}$

${ }^{I}$ Department of Environmental Biology, University of Rome, Sapienza, $P$. le Aldo Moro, 5, Roma, 00185, Italy; ${ }^{2}$ Consiglio per la Ricerca in Agricoltura e l'Analisi dell'Economia Agraria, Centro di Ricerca Difesa e Certificazione, Via C.G, Bertero 22, Rome, 00156, Italy; ${ }^{3}$ SARA EnviMob srl, via dei Castani 116, Rome, Italy. E-mail: luigi.faino@uniromal.it

Xylella fastidiosa $(X f)$ is a bacterial plant pathogen recently introduced in Europe. Subspecies and Sequence Type (ST), performed by Multi-Locus Sequence Typing (MLST) analysis, is mandatory for identifying new $X f$ outbreaks. In order to speed-up and simplify detection and identification of $X f$ in planta, we set-up a procedure based on MinION device for sequencing gDNA directly from infected or putatively-infected plant material and subsequent bioinformatic analysis. We analyze naturally infected plant species. The results on direct gDNA sequencing show that we could detect $X f$, even if for some samples we had few reads belonging to 
$X f$. Specifically, in-depth analysis showed that $X f$ can be detected in a reliable manner only in severely infected samples. In order to increase sensitivity, we add an amplification step prior the sequencing with MinION. According to the EPPO Standard PM7/24(4), we use the bacterial genes cys $G$ and malF for subspecies identification through amplicon sequencing. The results showed that with amplicon sequencing data analyzed by our bioinformatic tool we can detect $X f$ and identify the specific subspecies within the plant gDNA independently by the level of infection. This method may achieve a sensitivity higher than real-time PCR. Finally, we determine $X f$ ST from naturally infected plants thought MLST amplicons, pooled and sequenced by MinION. Using a custom python script, we reconstruct all seven housekeeping genes for each sample and assign them to a specific ST. Our system provides the possibility within few hours to detect and identify $X f$ in plant material at low level of infection.

\section{Trichoderma harzianum INAT11 elicits systemic resistance in maize against Fusarium verticillioides and Fusarium graminearum}

\section{Ferrigo, C. Scopel, M. Mondin, R. Causin, A. Raiola}

Dipartimento Territorio e Sistemi agro-forestali (TeSAF), Università di Padova, Agripolis, Viale dell'Università 16, 35020 Legnaro, PD, Italy. Email: davide.ferrigo@unipd.it

Pink Ear Rot (PER) and Red Ear Rot (RER) are common fungal diseases of maize mainly caused by Fusarium verticillioides and Fusarium graminearum, respectively. Ear rots result in yield losses and reduced kernel quality due to contamination by mycotoxins. Since no chemical treatments are available to control Fusarium disease in maize, biological control could represent a promising sustainable strategy. A commercial strain of Trichoderma harzianum (INAT11) was evaluated as seed treatment to manage PER and RER through induction of a systemic resistance response in maize. Infection trials on maize plants under controlled greenhouse conditions showed the capacity of INAT11 when applied to seed to reduce both $F$. verticillioides and $F$. graminearum disease incidence (37\% and $18.5 \%$, respectively) and severity (33\% and $28 \%$, respectively). The expression of some genes belonging to both ISR (LOX10, AOS, HPL and OPR8) and SAR pathways (PAL and PR1), and also a stress-related gene (POX), were analysed in the hours following infection by quantitative real-time PCR. In treated plants during Fusarium infection some ISR and SAR markers were upregulated. The up-regulation occurred at different levels likely related to their pathogenic lifestyles.

\section{Natural compounds assessment for eco-sustainable FHB control strategy}

\section{S. Francesconi, G.M. Balestra}

Dipartimento di Scienze Agrarie e Forestali (DAFNE), Università degli Studi della Tuscia, Via San Camillo de Lellis, snc, 01100, Viterbo, Italy. Email:balestra@unitus.it

Fusarium Head Blight (FHB) of wheat is one of the most devastating cereal disease. The causal agents belong to the Fusarium graminearum Species Complex (FGSC), causing head bleaching, necrosis, shrivelled kernels and mycotoxins accumulation. FHB control strategies are mainly based on synthetic chemicals applications during the flowering stage.
Chemicals belonging to Imidazole and Strobilurin are mainly applied to contrast FHB pathogens, but, because their efficiency is highly variable and they are toxic for human, animal and environment, efficient new ecofriendly approaches must be developed. In this study, we evaluated in vitro and in vivo anti-fungal activity of different molecules (gallic, ellagic, caffeic acids, chitosan hydrochloride, lignin, oleuropein and esculetin), in single and in combinations, at different concentrations $(0.01 \%-1 \%)$. In vitro assays were the following: 96 wells microtiter plate, incorporation, agar diffusion and volatile diffusion assays. Moreover, five different in vivo assays were performed in order to evaluate a putative phytotoxic activity and efficiency against pathogen artificial inoculation. Furthermore, a Real-Time qPCR was performed in order to understand the role of promising natural compounds on pathogens related genes regulation. Chitosan hydrochloride $0.5 \%$ resulted to be the most versatile and efficient compound, since it was able to control both spores multiplication and mycelium development, while esculetin and oleuropein resulted to inhibit mycelial growth through volatile diffusion; esculetin showed to reduce roots development. Promising results highlighted that a combination of chitosan hydrochloride and oleuropein is able to contrast $F$. graminearum infection and diffusion as much as chemical controls. Research financed by Rural Development Program (PSR) Regione Umbria 16.2 "SMART AGRI PLATFORM" Project

\section{Phenomics analysis and risk prediction of FHB}

\section{S. Francesconi ${ }^{1}$, M. Maesano ${ }^{2}$, F.V. Moresi $^{2}$, A. Harfouche ${ }^{2}$, G.M. Balestra $^{1}$}

${ }^{1}$ Dipartimento di Scienze Agrarie e Forestali (DAFNE), Università degli Studi della Tuscia, Via San Camillo de Lellis, snc, 01100, Viterbo, Italy; ${ }^{2}$ Dipartimento per l'Innovazione nei sistemi Biologici, Agroalimentari e Forestali (DIBAF), Università degli Studi della Tuscia, Via San Camillo de Lellis, snc, 01100, Viterbo, Italy.E-mail: balestra@unitus.it

Fusarium Head Blight (FHB) is a disease of small grain cereals reducing grain yield and quality. FHB incidence and severity vary with cereal growing areas, weather conditions and host plant resistance. Triticum durum results to be much more susceptible than $T$. aestivum and FHB control strategies are focused on using chemicals during the flowering stage. Estimation of FHB risk and early detection in field are important goals since they can help to reduce and optimize fungicide treatments. Based on the pathogen epidemiological stages we adapted an existing decision support system model to monitor FHB in Umbria Region. The information have been organized in a webbased system, called SMARTAGRI, able to generate five FHB risk levels based on climate and agronomic information of four partner farms in Umbria. In order to establish an innovative FHB early detection method, an experimental plot of T. durum cv. Marco Aurelio located in Amelia was subjected to thermal and RGB imaging in association with an Unmanned Aerial Vehicle (UAV), plant sampling to confirm pathogen presence, in-field spike temperature measurements and photosynthesis efficiency evaluations. After mosaicking, orthorectifing and georeferencing, the first in-field trial revealed the "leopard spot" FHB distribution and an increasing in temperature value in infected spikes, probably due to differential stomatal conductance regulation. A real-time qPCR will be performed on a series of 20 key genes in order to assess the role of stomatal conductance in FHB resistance and a putative differential regulation between infection status and drought stress status.

Research financed by Rural Development Program (PSR) Regione Umbria 16.2 "SMART AGRI PLATFORM" Project 
Believing is seeing: lessons from emerging viruses in grapevine

\section{Fuchs}

Cornell University, Plant Pathology and Plant Microbe-Biology Section, School of Integrative Plant Science, Cornell AgriTech, 15 Castle Creek Drive, Geneva, NY 14456, USA. E-mail: marc.fuchs@cornell.edu

With more than 80 different viruses identified so far, Vitis spp. is the plant species that hosts the most viruses. The occurrence of a multitude of viruses in this widely-grown fruit crop is likely explained by (i) a very long history of domestication and coexistence, (ii) a sparsity of resistance sources in Vitis spp., and (iii) an extensive exchange of germplasm on a global scale. Among the viruses of economic importance that were recently identified are grapevine Pinot gris virus (GPGV), grapevine vein clearing virus (GVCV) and grapevine red blotch virus (GRBV). These three viruses have emerged in the past decade as serious threats to the wine and grape industry. Although they have likely been present in grapevines for a long time, their detrimental effect on vine growth and production only became noticeable once introduced into the plant propagation material, and subsequently into commercial vineyards. Early on, symptoms associated with GPGV, GVCV and GRBV were deceiving due to similarities with symptoms caused by well-known viruses such as those involved in fanleaf degeneration or leafroll diseases. This clearly delayed their identification and the development of specific diagnostic tools. Eventually, efforts to characterize GPGV, GVCV and GRBV provided insights into their distribution, population structure, evolution, and unique epidemiological features that sets them apart from other commonly occurring grapevine viruses. Information on the biology and ecology of these three emerging viruses will be presented and discussed, and lessons learned during their discovery will be reviewed as they elegantly underscore how believing is seeing.

\section{A lignin-rich extract from Arundo donax protects zucchini plants from Pythium sp. attack}

\section{S. Galletti ${ }^{1}$, S. Cianchetta ${ }^{1}$, R. Roberti ${ }^{2}$}

${ }^{1}$ CREA - Research Centre for Agriculture and Environment, Via di Corticella 133, 40128 Bologna, Italy; ${ }^{2}$ Department of Agricultural and Food Sciences, University of Bologna, Viale G. Fanin 40, 40127 Bologna, Italy. E-mail: stefania.galletti@crea.gov.it

The treatment of the dry biomass of Arundo donax with an alkali, like $\mathrm{KOH}$, in a hot water solution $(10 \%$ A. donax powder in $\mathrm{KOH} 1.6 \%)$ gives rise to both de-lignified fibers, exploitable in biorefinery contexts, and a liquid phase, rich of lignin and nutrients (extract). This extract, properly neutralized, could be utilized as a soil improver with antifungal properties, that were highlighted in our previous in vitro experiments. Thus, an in vivo test on zucchini was carried out, under controlled conditions, to investigate the possible protective effect of a pre-sowing treatment with the extract $(200 \mathrm{ml} / 1$ of substrate) against Pythium sp., artificially inoculated into the substrate. The extract significantly reduced the inoculum concentration of the pathogen in peat and in sand and it effectively controlled the disease on zucchini by increasing plant emergence and total dry weight with respect to the not treated control, in sand. The extract reduced the germination percentage of zucchini seeds on filter paper, but no phytotoxic effects were found in vivo. This could be due to dilution effects and to partial adsorption of possible inhibitors, like polyphenols, by the substrate. This adsorption was demonstrated by spectrophotometric analysis of the leachate collected after watering the treated substrate, in comparison to the whole extract used for the treatment. The lignin-rich extract of $A$. donax seems to show interesting potentialities as an ingredient for innovative products to be used in sustainable agriculture, for the antifungal effects against telluric pathogens, like Pythium sp.

This work was partially supported by MIPAAF, D.D. n. 26329, AGROENER project, 2016

\section{Susceptibility of species and cultivars belonging to Pelargonium spp. to Verticillium nonalfalfae}

\author{
A. Garibaldi ${ }^{1}$, D. Bertetti ${ }^{1}$, M.L. Gullino ${ }^{1,2}$ \\ ${ }^{1}$ Università degli Studi di Torino, Centro AGROINNOVA, Largo Braccini 2 - \\ 10095 Grugliasco (TO), Italy; ${ }^{2}$ Università degli Studi di Torino, DISAFA, \\ Largo Braccini 2 - 10095 Grugliasco (TO), Italy. E-mail: \\ marialodovica.gullino@unito.it
}

Verticillium albo-atrum was recently divided into three new species: $V$. albo-atrum sensu stricto, V. alfalfae and V. nonalfalfae. This last has been reported on Pelargonium grandiflorum "Fabiola" for the first time in Italy. Successively, the susceptibility of 31 cultivars belonging to $P$. grandiflorum, $P$. peltatum and $P$. zonale was tested to this pathogen. Two single-spore strains of $V$. nonalfalfae from $P$. grandiflorum were grown in Potato Dextrose Broth (PDB) to obtain the conidial suspensions used in the tests. The roots of the rooted cuttings of tested cultivars were dipped in the conidial suspensions, while controls were dipped in sterile water. Then, all the plants were transplanted in containers, into a steam disinfested soil and maintained under greenhouse, at average daily temperatures ranging from 17.1 to $31.3^{\circ} \mathrm{C}$. The severity of Verticillium wilt on inoculated Pelargonium spp. was evaluated by counting and removing dead plants. At the end of the trials, the final evaluation of symptoms permitted to give a disease index (D.I.) to each tested cultivar and to assign it to one of the following classes: $\mathrm{R}=$ Resistant (D.I. 0-5); $\mathrm{PR}=$ Partially Resistant (D.I. 5.1-20); MS=Moderately Susceptible (D.I. 20.1-50); S=Susceptible (D.I. 50.1-75); HS=Highly Susceptible (D.I. 75.1-100). "Aristo Velvet Red", "Elegans Adriana", "Elegans Sunrise", "Elegans Rosanna", "Elegans Royalty White" and "Top Wicky", all belonging to $P$. grandiflorum, were the most susceptible cultivars. Most cultivars of $P$. peltatum and $P$. zonale were more or less susceptible to the pathogen, although "Decora Red", "Large Salmon", "Large White" and "Mexikanerin" were partially resistant.

\section{Genome sequence resource for Stemphylium vesicarium, causing Brown Spot disease of Pear}

\section{K. Gazzetti ${ }^{1}$, E.L. Diaconu ${ }^{2}$, I.M. Nanni ${ }^{1}$, A. Ciriani ${ }^{1}$, M. Collina ${ }^{1}$ \\ ${ }^{1}$ University of Bologna, Department of Agricultural and Food Science, Viale G. Fanin 42, 40127 Bologna, Italy; ${ }^{2}$ Bio-Fab Research srl-Via Mario Beltrami, 5 -00135 Roma.E-mail: marina.collina@unibo.it}

Stemphylium vesicarium (Wallr.) E. Simmons can cause several plants disease, as well the Brown Spot of Pear (BSP), which is one of the most economically important fungal disease in Europe. The knowledge of the $S$. vesicarium genome sequence and properties could indirectly improve the integrated control of BSP and of other plant infections. Genomic DNA was extracted from the full sensitive monoconidial strain 1731a13FI1M3, isolated from pear, then de novo sequenced by shotgun 
on Illumina Miseq platform. 1,127 contigs were assembled, obtaining a total assembly length of $38.66 \mathrm{Mb}$. Size is similar to those of Stemphylium lycopersici, the only other genome currently available for the Stemphilium genus. Gene prediction resulted in 12,309 putative genes. A primary functional annotation provided information about Orthologous Groups, Gene Ontology terms, KEGG pathways, and SMART/Pfam domains for each group. Furthermore, combined prediction of transmembrane topology and signal peptide were carried out, and genome sequence was also analyzed for the automatic genomic identification and analysis of biosynthetic gene clusters. Among predicted genes it was possible to identify several orthologues of genes required in other Ascomycota for pathogenesis or full virulence on plants. Moreover, the availability of genome sequence allowed to recover the orthologous of genes associated with signal transduction, the entire sequence coding for cyt $b$, and the succinate dehydrogenase subunits. The resource opens a new scenario in the investigation of fungal lifestyle and molecular plant/pathogen interaction, and could be fundamental in order to design more effective and sustainable fungicides management strategies to control the disease.

\section{Effect of leaf age on in vitro mycelial growth of Hymenoscyphus fraxineus}

\author{
L. Ghelardini ${ }^{1,2}$, C. Aglietti ${ }^{1}$, F. Cantini ${ }^{1}$, N. Ferretti ${ }^{1}$, N. Luchi ${ }^{2}$, G. \\ Marchi $^{1}$
}

${ }^{1}$ Department of Agriculture, Food, Environment and Forestry (DAGRI), University of Florence, Piazzale delle Cascine 28, 50144 Firenze, Italy; ${ }^{2}$ Institute for Sustainable Plant Protection (IPSP), CNR, Via Madonna del Piano 10, 50019 Sesto fiorentino, Italy.E-mail: luisa.ghelardini@unifi.it

Hymenoscyphus fraxineus, the alien fungus causing dieback on Fraxinus excelsior and Fraxinus angustifolia across Europe, was reported in the Northern Apennines in 2015. Correlations between ash leaf phenology and susceptibility to $H$. fraxineus were repeatedly observed, more resistant genotypes showing earlier leaf emergence and senescence. Leaf is the primary infection site for air-dispersed ascospores that penetrate blades, enter rachis vessels, reach twig parenchyma rays and kill cortex and cambium. On leaf colonization speed depends the pathogens' capacity to enter perennial organs before leaf shedding, and disease spread efficiency by aerial inoculum. In order to investigate the capacity of leaves to support fungal growth at different times during the season, fungal isolates from the Apennines focus were cultivated on agar media enriched with extracts from leaves of increasing age sampled from a healthy $F$. excelsior of local origin between May and October. In vitro growth varied depending on leaf age. For most isolates, the growth rate was minimum when leaves had just emerged from buds, reached a maximum on fully expanded leaves and was drastically reduced from mid August onwards. A few isolates however maintained an unchanged growth rate on media containing late summer and autumn leaves. In conclusion, if on the one hand disease spread in the Apennines is limited by scarce leaf receptivity in late summer and autumn (where local weather should favor fungal sporulation), on the other hand natural selection of the rare fungal genotypes able to efficiently grow on older leaves might partly overcome this limitation.

\section{Identification of Fusarium species occurring on several cultivars of soft and durum wheat from different areas of Tuscany}

V. Ghionna ${ }^{1}$, M. Haidukowski ${ }^{1}$, M.T. Cimmarusti ${ }^{1}$, S. Somma ${ }^{1}$, L. Mugnai, G. Carella ${ }^{2}$, M. Nocentini ${ }^{2}$, A. Salerno ${ }^{2}$, M. Masiello ${ }^{1}$, A.F. Logrieco $^{1}$, A. Moretti ${ }^{1}$
${ }^{1}$ Institute of Sciences of Food Production, National Research Council of Italy, Bari, Italy; ${ }^{2}$ Department of Agricultural, Food, Environmental and Forestry Sciences and Technologies, Florence, Italy. E-mail: veronica.ghionna@ispa.cnrit

Cereals are the main source of food for humans and animals. Among diseases affecting these crops, Fusarium Head Blight (FHB) of wheat is very worrisome since is caused by a complex of Fusarium species that can produce a wide range of harmful mycotoxins (MTX). These MTX can be accumulated in the kernels and represent a serious health issue for consumers. Moreover, each species is provided with a specific MTX profile, making very important a reliable identification of the species occurring in the ears for the correct evaluation of the potential toxicological risk of contaminated kernels. Therefore, monitoring is often carried out in order to evaluate the real contamination associated to Fusarium species. In recent years, a growing interest toward ancient wheat varieties was observed, and most of them showed to have high tolerance to FHB. In our study, 141 samples of ancient wheat cultivars (19 cvs. of soft wheat and 21 cvs. of durum wheat) were collected from several areas of Tuscany (Italy) during a period of five years (2013-2017) and analyzed for MTX content by high performance liquid chromatography (HPLC). The most frequently occurring MTX was deoxynivalenol (DON), occurring in $21 \%$ of durum and $52 \%$ of soft wheat samples. A wide variability of Fusarium species was identified, based on their morphological features and confirmed by sequencing translation elongation factor $1 \alpha$ gene, being $F$. poae, $F$. avenaceum, $F$. graminearum, $F$. equiseti and $F$. acuminatum the most important species associated to FHB of wheat in Tuscany.

\section{New ecofriendly antimicrobial peptides to control rice blast disease and Fusarium Head Blight}

R. Govind ${ }^{1}$, R. Caracciolo ${ }^{1}$, A. Quarantin ${ }^{1}$, A. Bolzonello ${ }^{1}$, S. Tundo $^{1}$, C. Castiglioni ${ }^{1}$, S. Odorizzi ${ }^{1}$, M. De Zotti ${ }^{2}$, F. Favaron ${ }^{1}$, A.M. Picco ${ }^{3}$, L.T. Do ${ }^{4}$, V.V. Vu ${ }^{5}$, H.M. Nguyen ${ }^{4}$, L. Sella ${ }^{1}$

${ }^{1}$ Department of Land, Environment, Agriculture and Forestry, University of Padova, Viale dell'Università 16, 35020, Legnaro (PD), Italy; ${ }^{2}$ Department of Chemistry, University of Padova, Via Marzolo 1, 35131 Padova, Italy; ${ }^{3}$ Department of Earth and Environmental Sciences, University of Pavia, Via S. Epifanio 14, 27100 Pavia, Italy; ${ }^{4}$ Duy Tan University, K7/25 Quang Trung, Da Nang, Vietnam; ${ }^{5}$ Nguyen Tat Thanh University, 298-300A Nguyen Tat Thanh Street, District 4, Ho Chi Minh City, Vietnam.E-mail: luca.sella@unipd.it

Analogs of the peptaibol Trichogin, a natural antimicrobial peptide produced by Trichoderma longibrachiatum, have been synthesized and tested in vitro against strains of Pyicularia oryzae, causal agent of the rice blast disease, and Fusarium graminearum, responsible of the Fusarium Head Blight (FHB) disease of cereals such as wheat. The screening has demonstrated that some peptide analogs are very effective in inhibiting spore germination and fungal growth at $\mu \mathrm{M}$ concentrations. These peptides have been tested also in planta to confirm their efficacy. Treatments performed on barley, a model plant for $P$. oryzae infections, and rice allowed to identify peptides with different efficacy in protecting leaves from blast symptoms. Besides, treatments of wheat spikes were able to strongly reduce FHB symptoms. To investigate the mode of action of these antimicrobial peptides, microscopy analyses have been performed. Peptide treatments cause a dense agglutination of the cytoplasm, that contracts from the spore cell wall, and induce auto-fluorescence of spores, which 
indicates that they are non-viable. Fluorescent microscopy performed with a peptide conjugated with a fluorophore showed that peptides are located intracellularly, in the agglutinated cytoplasm.

This project is part of the Scientific and Technological Cooperation Agreement between Italy and Vietnam and is funded by the Italian Ministry of Foreign Affairs and International Cooperation.

The genome of an Italian strain of Fusarium verticillioides: Nanopore - Illumina hybrid assembly

\section{A. Grottoli ${ }^{1,2,3}$, L. Faino ${ }^{2}$, M. Beccaccioli ${ }^{2,3}$, V. Scala ${ }^{4}$, M. Reverberi $^{2,3}$, C. Fanelli ${ }^{2,3}$ \\ ${ }^{1}$ Dipartimento di Scienze Agrarie e Forestali, Università degli Studi della Tuscia, Via San Camillo de Lellis snc 01100 Viterbo Italia; ${ }^{2}$ Dipartimento di Biologia Ambientale e dello Sviluppo, Sapienza, Università di Roma, P.le A. Moro, 00185 Roma, Italy; ${ }^{3}$ SARA EnviMob s.r.l., Via dei Castan, 116, 00172 Roma, Italia; ${ }^{4}$ Consiglio per la Ricerca in Agricoltura e l'Analisi dell'Economia Agraria, Centro di Ricerca Difesa e Certificazione, Via C.G, Bertero 22, Roma, 00156, Italia. E-mail: grottoliphd@gmail.com}

Fusarium verticillioides $(F v)$ is considered one of the most common plant pathogenic fungi affecting Zea mays (maize) roots, stalk tissues and kernels causing diseases, such as stalk and ear rot. $F v$ is capable to produce mycotoxins, including fumonisins, which can accumulate into the kernels in field as well as in storage that can be dangerous for animal or for human health. $F v$ is a species included in the Fusarium fujikuroi species complex (FFSC), the largest Fusarium species complex. FFSC species may have in common distinct phenotypic traits like mycotoxin production, host-specificity and supernumerary chromosomes (SCs) in addition to core chromosomes. These SCs may differ among isolates in presence/absence, length and gene-abundance and often play an important role in the biology of the pathogenic species in the complex. Here we report the first release of the genome of an Italian $F v$ strain (ITEM 10027). The assembly was made using both Oxford Nanopore Technologies MinION long reads and Illumina ${ }^{\circledR}$ short reads; the genes were annotated using RNAseq data. $F v 10027$ genome marks significant differences with the $F v$ published genome belonging to the strain 7600 isolated in USA. These striking dissimilarities account for the presence of genes shared with several Fusarium species, including F. fujikuroi.

\section{Anthracnose of aromatic and ornamental Lamiaceae in Italy, a case study of Colletotrichum species diversity}

\author{
V. Guarnaccia ${ }^{1,2}$, I. Martino $^{2}$, G. Gilardi ${ }^{1}$, M.L. Gullino ${ }^{1,2}$, \\ A. Garibaldi ${ }^{1}$ \\ ${ }^{1}$ Center of Competence Agroinnova, University of Torino, Largo Paolo \\ Braccini 2, 10095, Grugliasco, TO, Italy; ${ }^{2}$ DiSAFA, University of Torino, \\ Largo Paolo Braccini, 2, 10095 Grugliasco, TO, Italy. ${ }^{2}$. E-mail: \\ vladimiro.guarnaccia@unito.it
}

Species of Colletotrichum are considered important plant pathogens, saprobes, and endophytes on a wide range of plant hosts. In Italy, several Colletotrichum species have been reported in greenhouse environment. In this study we explored the occurrence, diversity and pathogenicity of Colletotrichum spp. associated with aromatic and ornamental plants belonging to the family Lamiaceae. Surveys were carried out during 20112018 in Liguria and Piedmont, Italy. A total of 20 Colletotrichum strains were isolated from symptomatic leaves and twigs of Ocimum basilicum (basil), Origanum vulgare (oregano) and different Salvia spp. A multilocus phylogeny was established based on four genomic loci (ITS, $G A P D H, A C T$ and TUB2) and the morphological characters of the isolates determined. Preliminary pathogenicity tests were performed with representative isolates. Colletotrichum strains were identified as members of three major species complexes: C. acutatum, C. destructivum and $C$. gloeosporioides. Colletotrichum fioriniae, C. bryonicola and $C$. fructicola were found in association with leaf lesions on Salvia leucantha, S. nemorosa and S. greggii, respectively. Colletotrichum coccodes was isolated from twig lesions of $S$. greggii. Moreover, $C$. fioriniae and $C$. ocimi were found as responsible of leaf anthracnose of oregano and basil, respectively. The pathogenicity of five representative isolates for each Colletotrichum species was tested on seedlings or rooted cuttings kept in a growth chamber. All the tested strains were pathogenic and reproduced symptoms identical to that observed in commercial greenhouses. The present study improves our understanding of Colletotrichum species associated with several hosts cultivated in Italy, and provides useful information for effective disease management.

This work was supported by funding from the European Union's Horizon 2020 research and innovation programme under grant no. 634179 'Effective Management of Pests and Harmful Alien Species - Integrated Solutions' (EMPHASIS)

\section{Effect of composts and BCAs on lettuce Fusarium wilt and on microbial communities}

M.L. Gullino ${ }^{1,2}$, A. Cucu ${ }^{1}$, G. Gilardi ${ }^{1}$, M. Pugliese ${ }^{1,2,3}$, A. Garibaldi ${ }^{1}$

${ }^{1}$ Università degli Studi di Torino, Centro AGROINNOVA, Largo Braccini 2 10095 Grugliasco (TO), Italy; ${ }^{2}$ Università degli Studi di Torino, DISAFA, Largo Braccini 2 - 10095 Grugliasco (TO), Italy; ${ }^{3}$ AgriNewTech srl, Via Livorno60-10144 Torino, Italy.E-mail: marialodovica.gullino@unito.it

Lettuce Fusarium wilt, caused by Fusarium oxysporum f. sp. lactucae, represents a major problem in most lettuce production areas worldwide. In the present study, two composts (a green compost and a microbial fortified compost) and different commercially available and experimental biological control agents (Bacillus subtilis QST713; Trichoderma gamsii ICC 012 + Trichoderma asperellum ICC 080; Trichoderma sp. TW2; Pseudomonas putida $\mathrm{FC} 7 \mathrm{~B}+\mathrm{FC} 8 \mathrm{~B}+\mathrm{FC} 9 \mathrm{~B}$ ) were applied, in a preventative way, in a naturally infested field experiment, against lettuce wilting, over two lettuce cultivar 'Novelsky' cropping seasons. The effects on rhizosphere and soil indigenous total microbial communities, nitrifiers and different functional genes (fungal chitinase, bacterial 2,4-diacetylphloroglucinol and HCN synthase) were also investigated. Composts, applied in nursery at $10 \%$ and in soil mixture before transplanting $\left(1 \mathrm{~kg} / \mathrm{m}^{2}\right)$, provided a consistent Fusarium wilt reduction from 60 to $75 \%$. The application of BCAs at the nursery level provided a disease reduction of $30 \%-78 \%$ in the field trials. Furthermore, the treatments improved the abundance of Bacillus sp., Trichoderma sp. and Pseudomonas sp. in the rhizosphere and bulk soil, while they did not influence negatively the nontarget microbial communities. The use of composts and BCAs 
appears as an effective and safe strategy to implement sustainable crop protection strategies.

This work was supported by the European Union's Horizon 2020 research and innovation Pogramme under grant agreement No. 633999, EUCLID EU-CHINA Lever for IPM Demonstration.

\section{Susceptibility of lettuce cultivars to Italian isolates of Fusarium oxysporum f. sp. lactucae Race 4}

\author{
M.L. Gullino ${ }^{1,2}$, G. Gilardi ${ }^{1}$, A. Garibaldi ${ }^{1}$ \\ ${ }^{1}$ Università degli Studi di Torino, Centro AGROINNOVA, Largo Braccini \\ 2 - 10095 Grugliasco (TO), Italy; ${ }^{2}$ Università degli Studi di Torino, \\ DISAFA, Largo Braccini 2 - 10095 Grugliasco (TO), Italy. E-mail: \\ marialodovica.gullino@unito.it
}

Race 4 of Fusarium oxysporum f. sp. lactucae is gradually spreading to new countries and, after being isolated in the Netherlands, Belgium, United Kingdom and Ireland, it has been recently detected in northern and southern Italy. Since the use of resistant cultivars represents the most effective practice to manage lettuce Fusarium wilt, the susceptibility of lettuce cultivars to Race 4 isolates of $F$. oxysporum f. sp. lactucae was tested. Repeated trials were carried out in greenhouse at temperatures between 26 and $30^{\circ} \mathrm{C}$. Forty cultivars, belonging to different lettuce types, were chosen among those commercially available. Seedlings (15-20-dayold plants) were artificially inoculated by root immersion in each conidial suspension at $1 \times 10^{6} \mathrm{CFU} / \mathrm{ml}$ and planted in $12 \mathrm{~L}$ pots filled with a peat medium, using 20 -to-30 plants/cultivar each trial. At the final evaluation the reaction of susceptibly was assigned to each cultivar. The most consistent results of a resistant or partially resistant reaction was observed for the cultivars Biondyne, Copacabana, Marilisa, Redin, Summerbell, Kireve, Cencibel, Flavus, Redsea, Ordino, Gloriole, and Lunabionda. The detection of Race 4 of the pathogen causes concern in Italy where growers usually choose cultivars of lettuce resistant to Race 1. Consequently, a careful monitoring of the race situation in the field is necessary for an efficient use of the genetic control measure.

This work was supported by the European Union's Horizon 2020 research and innovation programme under grant agreement No. 633999, EUCLID EU-CHINA Lever for IPM Demonstration

Assessment of the endophytic fungal community in Apulian olive varieties with different potential susceptibilities to Xylella fastidiosa

\author{
A. Hanani ${ }^{1,2}$, F. Valentini ${ }^{1}$, S.M. Sanzani ${ }^{1}$, M. Gallo ${ }^{1}$, S.W. Davino ${ }^{2}$, \\ A.M. D'Onghia ${ }^{1}$ \\ ${ }^{1}$ CIHEAM - Istituto Agronomico Mediterraneo di Bari, Via Ceglie, 9, \\ I-70010, Valenzano, BA-Italy; ${ }^{2}$ Department of Agricultural, Food and \\ Forest science University of Palermo, Palermo, Italy. E-mail: \\ arafat.samra@gmail.com
}

The Apulian olive culture has been seriously affected by the arrival of Xylella fastidiosa subsp. pauca strain ST53. In the outbreak area, different susceptibility to the infection was found among the olive varieties. In particular, the cv. Leccino has showed mild symptoms and low bacterium concentration compared to the susceptible cv. Ogliarola. With the aim to deepen the knowledge on the different susceptibility of olive cultivars to $X$. fastidiosa, this study was built to identify, characterize and quantify seasonally the endophytic fungal community from the twig sap of infected and not infected olive cultivars, grown in different Apulian phytosanitary demarcated areas. During 2018-2019, different fungal species were isolated from the twig sap of Apulian olive cultivars using the CIHEAM patent extraction SAP and the tissue print methodologies. Nineteen fungal isolates were characterized by morphological and molecular approaches. Macro- and microscopic observations and sequencing of internal transcribed spacer (ITS) region of those isolates showed significant seasonal diversity within fungal community. In spring samples, isolates belonging to the ubiquitous genera Penicillium, Aspergillus and Cladosporium were predominant. Whereas, other fungi belonging to the wood decay genera Cytospora and Ceuthospora were present at a lower extent. In summer samples, further putative pathogens as Fusarium sp., Pseudophaeomoniella oleae (CoDiRO agent), and Stigmatodiscus enigmaticus were mainly found. Furthermore, putative antagonists belonging to the genus Paraconiothyrium were recorded. Notably, these latter might interact antagonistically towards bacterial growth.

\section{Characterization of Fusarium proliferatum isolates from garlic in Italy}

A. Infantino ${ }^{1}$, S. Campagna ${ }^{2}$, G.M. Palmaccio ${ }^{2}$, M. Beccaccioli ${ }^{2}$, M. Aragona $^{1}$, C. Costa ${ }^{3}$, M. Reverberi ${ }^{1}$

${ }^{1}$ CREA-DC of Rome, Research Center for Plant Protection and Certification, Via C.G. Bertero 22, 00156 Roma, Italy; ${ }^{2}$ Sapienza University of Rome, Dept. Environmental Biology, Piazzale Aldo Moro 5, 00185 Roma, Italy; ${ }^{3}$ CREA-IT, Research Center for Engineering and Agro-Food processing, Via della Pascolare 16, 00015 Monterotondo (RM).E-mail: alessandro.infantino@crea.gov.it

Fusarium rot of garlic (Allium sativum L.) is of concern in many garlic cultivation areas worldwide, including Italy. Fusarium proliferatum has been identified as the species mostly involved in the disease. This species has a broad host range, including many cultivated and ornamental crops, like wheat, rice, maize, palm. At CREA DC, a collection of 32 isolates from symptomatic garlic bulbs and from other cultivated species has been established. The identification has been done by morphology and by PCR using specific primers. Mating type has also been evaluated by PCR. A detached bulbil inoculation method has been developed on garlic for the characterization of the pathogenicity of the isolates. Isolates from garlic were the most virulent, even if isolates from other species (i.e. wheat) were able to induce symptoms similar to those produced by isolates from garlic. Mycotoxin production of isolates grown for 4 weeks on autoclaved rice kernels has been evaluated by HPLC-MS/MS. Five mycotoxins have been determined: fusaric acid (FA), beauvericin (BEA), fumonisin (FB1 and FB2), and moniliformin (MON). Among them BEA was the most represented. The possible correlation among virulence, growth rate, host, mating type, geographic origin and mycotoxin production is under evaluation. The use of healthy bulbs and long rotations with crops not susceptible to $F$. proliferatum are important strategies for the control of the disease.

Olpidium bornovanus and $O$. virulentus as melon pathogens causing root rot and vine decline in Latium and Sardinia

A. Infantino ${ }^{1}$, V. Balmas ${ }^{2}$, S. Mocali ${ }^{3}$, C. Chiellini ${ }^{3}$, D. Martignoni ${ }^{4}$, M.P. Aleandri ${ }^{4}$, L. Tommasoli ${ }^{1}$, A. Haegi ${ }^{1}$, R. Reda ${ }^{4}$, G. Chilosi ${ }^{4}$

${ }^{1}$ Consiglio per la Ricerca in Agricoltura e l'Analisi dell'Economia Agraria (CREA), Centro di Ricerca per la Patologia Vegetale, 00156 Roma, Italy; ${ }^{2}$ Dipartimento di Agraria, Università degli Studi di 
Sassari, 07100 Sassari, Italy; ${ }^{3}$ Consiglio per la Ricerca in Agricoltura e l'Analisi dell'Economia Agraria (CREA), Centro di ricerca per l'agrobiologia e la pedologia, 50125 Firenze, Italy; ${ }^{4}$ Dipartimento per l'Innovazione dei Sistemi Biologici, Agroalimentari e Forestali, Università degli Studi della Tuscia, 01100 Viterbo, Italy. E-mail: chilosi@unitus.it

Root rot and vine decline (RRVD) has become one of the most important diseases of melon. Recent evidences indicate that Monosporascus cannonballus is not the sole cause of RRVD, but Olpidium spp. are also involved. The aim of this study was to ascertain the potential concomitant involvement of these pathogens in the development RRVD in farms in Maremma Laziale (North Latium) (in greenhouse) and Central Sardinia (open field) with a history of the disease. In Maremma Laziale, M. cannonballus was the preponderant pathogen isolated from symptomatic roots. Roots from bait melon plants grown in contaminated soil were colonized by both $O$. bornovanus and $O$. virulentus and by M. cannonballus, showing roots browning and foliage wilt. In the tripartite pathogenicity test, $O$. bornovanus alone was found to be a virulent pathogen, capable of colonizing roots resulting in root browning and foliage wilting. In Sardinia, samples were collected in two sites, Sassu and Sinis (Oristano). In both surveyed sites, Plectosphaerella melonis was the most frequent fungal species isolated from collapsed roots, M. cannonballus was recovered from plants from Sinis, but not from those from Sassu. O. bornovanus was detected in roots of bait plants from Sassu, and both Olpidium species in those from Sinis. Our results confirmed that $O$. bornovanus likely assumes a key role in association not only with $M$. cannonballus also with $P$. melonis. NGS analysis, performed using soils from both sites indicated that difference in pathogenetic pattern may be associated also to variation in soil fungal community composition and function profile.

\section{The Edge of Tomorrow - Plant Health in the $21^{\text {st }}$ Century S. Kamoun}

The Sainsbury Laboratory, Norwich Research Park, Norwich, United Kingdom.E-mail: sophien.kamoun@tsl.ac.uk

Infectious plant diseases cause havoc to world agriculture and threaten to slow laudable efforts to launch a second green revolution to meet the food security needs of a booming world population. Filamentous pathogens such as the rice blast fungus, wheat stripe and stem rust, the Irish potato famine pathogen, and many others continue to trigger recurrent epidemics with far reaching consequences. In this talk, I will discuss how it is possible to perform cutting-edge research and significantly advance knowledge on economically important pathosystems, particularly in the post-genomics era. I will focus on the blast fungus Magnaporthe oryzae, a devastating cereal killer that infects the crops wheat, barley and rice, which are staple food for a majority of the world population. I will discuss our personal experience with the appearance in Bangladesh of wheat blast and stress the importance of open-science platforms and crowdsourced community responses in tackling emerging plant diseases. Also, together with several collaborators, we gained an unprecedented level of detail of the molecular interactions that define host-pathogen recognitions by solving the crystal structures of effectors of the blast fungus in complex with plant proteins and reconstructing the evolutionary history of these molecular interactions. Our aim is to build on these discoveries to drive both basic and applied plant pathology. We have started to develop a thorough understanding of the biophysical properties of pathogen effector binding to host proteins and their consequences on pathogenesis and immunity. Such knowledge, along with related mechanistic and evolutionary studies, will guide the retooling of the plant immune system towards resistance to diseases. Ultimately, we will deliver traits and nontransgenic cultivars for breeding disease resistance in crops.

\section{Seed transmission of tomato leaf curl New Delhi virus Italian isolate in zucchini squash}

\author{
E.-J. Kil ${ }^{1}$, T.B.T. Vo ${ }^{1}$, C. Fadhila ${ }^{1}$, A. Lal $^{1}$, H.T. Phuong ${ }^{1}$, E. \\ Troiano $^{2}$, G. Parrella ${ }^{2}$, S. Lee ${ }^{1}$
}

${ }^{1}$ Department of Integrative Biotechnology, Sungkyunkwan University, Suwon 16419, Korea; ${ }^{2}$ Istituto per la Protezione Sostenibile delle Piante, CNR, Portici 80055, Italy.E-mail: meitantei7@skku.edu

Tomato leaf curl New Delhi virus (ToLCNDV) is a bipartite begomovirus affecting tomato cultivation in Indian subcontinent. Recently, however, ToLCNDV has spread into Mediterranean countries including Italy, and occurred in cucurbits causing economic damages. Although ToLCNDV is known to be transmitted by sweet potato whitefly (Bemisia tabaci), like other begomoviruses, it is not clear how ToLCNDV has suddenly spread from the Indian subcontinent to the Mediterranean region. In 2017, ToLCNDV was diagnosed in young seedlings germinated naturally from fruits fallen last year in a farm located in Giugliano in Campania, Naples, Italy where ToLCNDV occurred. Because lots of sweet potato whiteflies were spread naturally in that region, it was necessary to identify that result in an artificial insect vector-free condition. Seeds were harvested from ToLCNDV-infected zucchini squash plants in Naples in 2017 and 2018 to examine whether ToLCNDV can be transmitted from zucchini squash seeds to young plants. Viral DNA was isolated from harvested seeds and 2-week-old seedlings germinated from them respectively, and amplified with a ToLCNDV-specific primer set. According to PCR results, viral contamination was confirmed from all harvested seeds and dissemination was proven from almost half of tested seedling samples (57.14-68.75\%). Virus infection was confirmed by organ-specific PCR from seedling samples. This is the first report demonstrating that ToLCNDV Italian isolate is a seed-transmissible virus in zucchini squash plants.

\section{Designing dual-active fungicides for simultaneous inhibi- tion of multiple target sites}

\section{A. Kunova, M. Zuccolo, F. Forlani, S. Dallavalle, P. Cortesi}

DeFENS - Department of Food, Environmental and Nutritional Sciences, Università degli Studi di Milano, via Celoria 2, 20133, Milano, Italy. E-mail: andrea.kunova@unimi.it

The repeated application of fungicides with different modes of action is indispensable in current disease management programs to effectively control diseases and to delay the development of resistance in fungal plant pathogens. Fungicides can be applied in alternation or together in a mixture, the latter can be developed by a manufacturer as a co-formulation or prepared by the user as a tank mix. Although there are numerous advantages of using fungicide mixtures, their preparation requires more expert knowledge about the composition of single components and their incompatibility may lead to the loss of their efficacy. To avoid these drawbacks, our aim was to combine two fungicides with different modes of action into a single molecule, preserving its dual activity against both molecular targets of single fungicides. In particular, we covalently joined the pharmacophores of strobilurin- and succinate dehydrogenase inhibitor (SDHI) fungicides with a linker to obtain novel hybrid molecules. The compounds were tested against important fungal plant pathogens and showed good inhibition of Pyricularia oryzae and Sclerotinia sclerotiorum with activity comparable to commercial fungicides. The 
inhibition of the cytochrome $b c l$ and the succinate dehydrogenase enzyme activities confirmed dual mechanism of action of the new molecules. The dual-active molecules will be further optimized to increase their binding and inhibition of the two targets.

\section{Recovery of Phytophthora parvispora from a Sicilian ripar- ian ecosystem}

F. La Spada ${ }^{1}$, F. Aloi ${ }^{1,2}$, M. Riolo ${ }^{1}$, A. Pane ${ }^{1}$, M. Evoli ${ }^{1}$, R. Faedda ${ }^{1}$, S.O. Cacciola ${ }^{1}$

${ }^{1}$ Department of Agricultural, Food and Environment (Di3A), University of Catania, via S. Sofia 100, 95123 Catania, Italy; ${ }^{2}$ Department of Agricultural, Food and Forest Sciences, University of Palermo, Viale delle Scienze, Building 4, 90128 Palermo, Italy. E-mail: olgacacciola@unict.it

In a recent survey, carried out in autumn 2017, Phytophthora parvispora has been recovered from Complesso Speleologico Villasmundo - S. Alfio Regional Natural Reserve (Hyblaean Mountains - south-eastern Sicily, Italy) at an altitude of about 100 meters a.s.l. from a chronically declining riparian forest along the course of a torrential stream. Isolates of the species have been obtained by using baiting techniques from rhizosphere soil of a symptomatic willow (Salix pedicellata) tree; the identification was carried out by combining morphological features with sequences of internal transcribed spacer regions (ITS1 and ITS2) and 5.8S gene. Originally identified as a variety of Phytophthora cinnamomi (i.e.: Phytophthora cinnamomi var. parvispora), Phytophthora parvispora has been associated to a severe dieback of Arbutus unedo in Sardinia, Italy, and described as a distinct species in the ITS clade 7a. In Sicily, it has been previously reported only from nurseries in association with cultivated Mandevilla spp. In view of its biological and ecological characteristics, $P$. parvispora can endure short periods of soil waterlogging and high relative humidity of the air alternating with long periods of drought, typical of the Mediterranean ecosystem where it has been found.

\section{Evaluation of olive knot disease and epiphytic bacterial population in a world-wide olive germplasm collection}

G. Licciardello ${ }^{1}$, S. Di Silvestro ${ }^{1}$, M. Sciara ${ }^{1}$, M.P. Russo ${ }^{1}$, G. Sorrentino ${ }^{1}$, M.C. Strano ${ }^{1}$, V. Catara ${ }^{2}$, P. Caruso ${ }^{1}$

${ }^{1}$ Consiglio per la Ricerca in agricoltura e l'analisi dell'Economia Agraria-Centro di ricerca Olivicoltura, Frutticoltura e Agrumicoltura (CREA), Corso Savoia, 190 Acireale (CT), Italy; ${ }^{2}$ Dipartimento di Agricoltura, Alimentazione e Ambiente (Di3A), Università degli Studi di Catania, Via Santa Sofia 100, 95130 Catania, Italy. Email:grazia.licciardello@crea.gov.it

Pseudomonas savastanoi pv. savastanoi (Psv), causal agent of olive knot disease which seriously affects olive production in the Mediterranean basin, has a resident phase on symptomless twigs and leaves surfaces that represent the major source of available inoculum for infections. In the framework of a project aimed at evaluating varietal susceptibility, we performed a survey in "Villa Zagaria" world-wide olive germplasm collection located near Enna (Sicily), to assess the incidence and severity of olive knot disease and to estimate PSv epiphytic bacterial populations. The visual inspection of 687 trees belonging to 290 varieties revealed an incidence of $97 \%$ of infections and a different disease severity (0-4 scale) among the varieties considered. Noteworthy, 'Montonica', 'Turdunazza' and 'Verdello grosso' (Sicily), 'Favarol' (Veneto), 'Cipressino' (Puglia), 'Negrera' (Liguria) and 'Ascolana' (Marche) did not shown any knot on twigs and branches. Epiphytic populations on twigs of 24 varieties showing different disease severity were evaluated both in winter and in late spring, by $P s v$-like colonies count on King's medium B and real-time PCR. No correlation was found between disease severity and $P s v$ population size. In spring $P s v$ concentration was about $17 \%$ higher than in winter ranging almost between $2 \times 10^{2}-2.3 \times$ $10^{4} \mathrm{cfu} \mathrm{m}^{-1}$. The presence of other bacterial species such as Pantoea agglomerans and Erwinia spp. was also evaluated. A collection of Psv isolates was set up for further characterization. Pathogenicity tests on detached leaves of symptomless and susceptible Sicilian varieties confirmed visual results. Stem inoculations on grafted plants are under evaluation.

The work has been financially supported by SALVAOLIVI project (MIPAAFT, DM 59 del 10/01/2018).

\section{Colletotrichum acutatum and C. kahawae subsp. ciggaro in olive fruits affected by anthracnose in Sicily}

\section{G. Licciardello, C. Strano, P. Caruso, R. Contarino, G. Sorrentino, S. Di Silvestro}

Consiglio per la Ricerca in agricoltura e l'analisi dell'Economia Agraria-Centro di ricerca Olivicoltura, Frutticoltura e Agrumicoltura (CREA), Corso Savoia, 190 Acireale (CT), Italy. E-mail: grazia.licciardello@crea.gov.it

Colletotrichum acutatum causes many of the world's most devastating diseases in different herbaceous crops and in a number of fruit trees. The fungus is quite spread in Portugal where severely affects oil production and quality. Since olive infected trees have been recently detected in Calabria, Puglia and Basilicata, a large survey for this pathogen has started in Sicily where six protected areas for denomination of origin (PDO) are designated. During a screening carried out in winter 2018 in a germplasm collection located near Enna (Sicily), typical symptoms of anthracnose (soft and dark brown rot, brown sunken lesions with emerging black or orange acervuli and partial mummification) were observed on 34 Sicilian ecotypes with a variable number of affected fruits (3-34\%). In order to confirm the diagnosis small pieces of tissues were placed aseptically on potato dextrose agar (PDA) amended with $100 \mu \mathrm{g} \mathrm{ml}^{-1}$ streptomycin. Single acervuli emerging from the olive fruits were also directly placed on PDA plates. Three colonies showing pink salmon mycelia and concentric zone of shiny orange conidial masses (P77, P185, B13) and one colony (Perg6B) with grey mycelium and black concentric zones, were further characterized by molecular analysis based on rDNA-ITS region (ITS) and $\beta$-tubulin 2 (TUB-2) gene. Nucleotide sequence analysis revealed that P77, P185 and B13 isolates belong to C. acutatum s.s., whereas Perg6B to C. kahawae subsp. ciggaro. To our knowledge this is the first report of $C$. acutatum and C. kahawae subsp. ciggaro in Sicily.

The work has been financially supported by SALVAOLIVI project (MIPAAFT, DM 59 del 10/01/2018).

\section{Characterization of Phytophthora and Botryosphaeriaceae species associated with emerging olive diseases in north- east Italy}

\section{B.T. Linaldeddu, C. Bregant, L. Montecchio, F. Favaron, L. Sella}

Dipartimento Territorio e Sistemi Agro-Forestali, Università degli Studi di Padova, Viale dell'Università 16, 35020 Legnaro (Italy). E-mail: luca.sella@unipd.it

Severe and unusual trunk and branch diseases of olive trees have recently been observed in several orchards in Veneto (Italy). Since there is little information about the aetiology of these diseases and given the high economic importance of these agro-systems, an in-depth study was carried out. From 
summer to autumn 2018, 230 symptomatic samples were collected from olive trees showing cankers and branch dieback as well as collar and root rot with basal phloem lesions extending up from below ground level in 19 olive growing areas. Based on morphology, colony appearance and DNA sequence data, eight Botryosphaeriaceae species belonging to four genera and four Phytophthora species were isolated and identified. These included Botryosphaeria dothidea, Diplodia mutila, D. olivarum, D. seriata, D. subglobosa, Dothiorella sarmentorum, D. sempervirentis and Neofusicoccum parvum (Botryosphaeriaceae), Phytophthora acerina, $P$. palmivora, $P$. pini and $P$. plurivora. Pathogenicity tests in potted olive trees completed Koch's postulates and confirmed that $N$. parvum and all the Phytophthora species investigated were capable to reproduce the symptoms observed in field conditions. Because of their aggressiveness, these five pathogens represent a serious risk for olive trees in northeast Italy; therefore, specific control measures need to be developed. The findings of this study showed that the diversity of olive pathogens is greater than previously recognised and further studies are necessary to determine their possible synergistic interaction. The species $D$. subglobosa, D. sempervirentis, $P$. acerina, P. pini and P. plurivora are here reported for the first time as olive pathogens. This work was supported by a project of the Veneto region (DGR $n .1582$ del 30/10/2018)

\section{Neonectria coccinea associated with canker and dieback of Fagus sylvatica in Italy}

\section{B.T. Linaldeddu, F. Contorno, L. Montecchio}

Dipartimento Territorio e Sistemi Agro-Forestali, Università degli Studi di Padova, Viale dell'Università 16, 35020 Legnaro (Italy). E-mail: benedetto.linaldeddu@unipd.it

The occurrence of severe branch canker and dieback symptoms on beech (Fagus sylvatica L.) was observed in a large monospecific beech forest in northeast Italy (Bosco del Cansiglio). Given the limited information available on this disease an investigation was carried out to evaluate its spread and incidence and to characterize the pathogenic species involved. Results of the survey showed the high diffusion of symptomatic beech trees with open cankers and branch dieback in all investigated sites. At the same time, the mechanical stability of many trees was compromised by the occurrence of several cankers in the branches and along the trunk. Isolations carried out from cankered branches yielded on potato dextrose agar (PDA) at $25^{\circ} \mathrm{C}$ white to orange fungal colonies, with an appressed and compact mycelium. On the basis of morphological features and DNA sequence data all isolates were identified as Neonectria coccinea (Pers.) Rossman \& Samuels. Pathogenicity trials carried out under laboratory conditions on wounded beech branches showed that $N$. coccinea is a beech pathogen. The results obtained in this study have allowed us to expand the knowledge about the bio-ecology of $N$. coccinea, a fungal species for which there are still many knowledge gaps. Further investigation on the natural corridors that can prevent and/or favour the spread and establishment of this pathogen are planned.

\section{Characterization of Phytophthora and Phytopythium spe- cies associated with ink disease of Castanea sativa in Italy}

\section{B.T. Linaldeddu, M. Farioli, L. Montecchio}

Dipartimento Territorio e Sistemi Agro-Forestali, Università degli Studi di Padova, Viale dell'Università 16, 35020 Legnaro (Italy). E-mail: benedetto.linaldeddu@unipd.it

Over the last decades an increasing number of Phytophthora species have been found to be involved in the chestnut ink disease aetiology in Europe.
Since little is known about the occurrence and spread of Phytophthora spp. in northeast Italy, a survey was carried out in four chestnut woodlands with trees showing typical ink disease symptoms. Isolations were made from both rhizosphere soil and root samples of symptomatic trees using fresh leaves of Quercus suber L. and Sambucus nigra L. as baits. Morphological and DNA based techniques were used to identify and characterize the isolates obtained. The survey revealed the presence of five species associated with symptomatic chestnut trees: Phytophthora acerina, P. cinnamomi, P. plurivora, Phytopythium mercuriale, and Pp. vexans. Phytophthora cinnamomi was the most widespread species in the investigate sites. Pathogenicity of all species was verified by living logs inoculation, under controlled laboratory conditions. All species were shown to be pathogenic on chestnut. Phytophthora acerina, Pp. mercuriale and $P$ p. vexans are here reported for the first time as chestnut pathogens. The results obtained emphasize that several species of Phytophthora and Phytopythium may represent a serious threat for chestnut woodlands.

Neofusicoccum parvum and Diplodia olivarum: the main species associated with a new disease of wild olive in Italy

\author{
B.T. Linaldeddu ${ }^{1}$, D. Manca ${ }^{1}$, L. Maddau ${ }^{2}$, C. Pinna ${ }^{2}$, L. \\ Montecchio ${ }^{1}$ \\ ${ }^{1}$ Dipartimento Territorio e Sistemi Agro-Forestali, Università degli Studi \\ di Padova, Viale dell'Università 16, 35020 Legnaro, Italy; ${ }^{2}$ Dipartimento \\ di Agraria, Università degli Studi di Sassari, Viale Italia, 39, 07100 \\ Sassari, Italy; E-mail: benedetto.linaldeddu@unipd.it
}

Wild olives (Olea europaea subsp. europaea var. sylvestris) represent a distinct botanical variety of the subspecies europaea naturally distributed throughout the Mediterranean region. In Sardinia (Italy), wild olive woodlands represent an important component of olive genetic heritage. Wild olive suffers from few major disease problems, however since 2017, a new and unusual disease leading to death young and old wild olive trees has been observed in several woodlands of high ecological value in the north-eastern part of Sardinia. Declining trees showed a variety of symptoms including branch dieback, leaf chlorosis and epicormic shoots on trunk and branches. The most frequent symptom observed consisted of sunken cankers on branches. These cankers often exuded a white to orange sap, giving them the appearance of bleeding, which gradually dried to a whitish gluey mass on the bark. Symptomatic woody samples yielded fungal isolates belonging to three distinct families: Botryosphaeriaceae, Pestalotiopsidaceae and Xylariaceae. Morphological and DNA sequence data revealed five distinct fungal species, namely Diplodia olivarum, Dothiorella sarmentorum, Neofusicoccum parvum, Pestalotiopsis hawaiiensis, and Rosellinia corticium. Pathogenicity trials carried out in field conditions on wild olive trees showed that all of the isolated species are pathogenic. Neofusicoccum parvum and Diplodia olivarum proved to be the most aggressive species. Our results provide the first evidence for a combined involvement of different Botryosphaeriaceae species in the aetiology of a new disease of wild olive in Italy.

\section{HIGS and SIGS assessment as control strategy for grape- vine downy mildew}

\section{L. $\mathrm{Ma}^{1}$, D. Gebremichael ${ }^{1}$, L. Capriotti ${ }^{2}$, S. Sabbadini ${ }^{2}$, B. Mezzetti $^{2}$, E. Baraldi ${ }^{1}$}

${ }^{1}$ University of Bologna, DISTAL (Department of Food and Agricultural Science), Viale Fanin 46, 40127 Bologna, Italy; ${ }^{2}$ Polytechnic University of Marche, D3A (Department of Agricultural, Food and Environmental Sciences), Ancona, Italy.E-mail: elena.baraldi@unibo.it 
Fungal pathogens deliver small RNAs into plant cells to silence host immunity genes, which are mostly produced by Dicer-like proteins. The host induced gene silencing (HIGS) and spray-induced gene silencing (SIGS) are efficient strategies of disease control and can be used to protect plants, however more information is needed to clarify the specificity and efficacy of dsRNAs molecules. HIGS and SIGS targeting fungal Dicer-like genes (necessary for fungal sRNA generation) have already been demonstrated to effectively act against pathogenic fungi during fruit infection. Downy mildew, caused by the oomycete Plasmopara viticola, is a major pathogen of grapevine currently controlled by agrochemical applications, raising social concern and pressure to develop alternative protection means. To assess HIGS and SIGS as alternative control methods against $P$. viticola, we produced dsRNA to silence Dicer-like genes of this pathogen by generating plasmid constructs with intron-spaced partial sense and antisense DNA sequences. Nicotiana benthamiana leaves were agroinfiltrated with these constructs to produce dsRNA molecules. These will be assessed on $P$. viticola infecting grape leaves. The same constructs have been used for genetic transformation of grape cv. Thompson seedless. The effect of RNAi in planta stable expression (HIGS) in inducing downy mildew resistance will be tested.

\section{Screening for resistance in grapevine germplasm and de- velopment of low-risk fungicides: an integrated research approach for the management of grapevine downy mildew}

G. Maddalena' ${ }^{1}$, G. De Lorenzis ${ }^{1}$, M. Brilli ${ }^{2}$, S. Masiero ${ }^{2}$, P. Pesaresi², A. Passera ${ }^{1}$, D. Maghradze ${ }^{3,4}$, O. Failla ${ }^{1}$, F. Quaglino ${ }^{1}$, S.L. Toffolatti $^{1}$

${ }^{1}$ Dipartimento di Scienze Agrarie e Ambientali - Produzione, Territorio, Agroenergia (DISAA), Università degli Studi di Milano, via Celoria 2 20133 Milano, Italy; ${ }^{2}$ Dipartimento di Bioscienze, via Celoria 26, Università degli Studi di Milano - 20133 Milano, Italy; ${ }^{3}$ Scientific Research Center of Agriculture, Marshal Gelovani Avenue 6, 0159. Tbilisi. Georgia; ${ }^{4}$ Faculty of Viticulture and Winemaking, Caucasus International University, Chargali Street 73, 0141, Tbilisi, Georgia. Email: silvia.toffolatti@unimi.it

The application of fungicides for the control of downy mildew, caused by the oomycete Plasmopara viticola, greatly contributes to make viticulture one of the less sustainable branches of agriculture. An optimal disease management, which is effective, economically feasible for the farmer and safe for human health and the environment, requires the integration of different strategies. New tools for the downy mildew management, based on selection of resistant cultivars and development of low-risk fungicides, were investigated. A resistant Vitis vinifera cultivar, named Mgaloblishvili, was found by screening a large collection of Caucasian germplasm. Based on comparative transcriptome analysis, unique traits related to candidate pathogen effectors and plant genes that are not expressed in common susceptible (Pinot noir) and resistant (Bianca) cultivars were found. One of the drawbacks of the cultivation of disease-resistant grapevine varieties is the selection of strains able to overcome plant resistance. In order to achieve a durable resistance, some treatments against $P$. viticola could be still needed. This highlights the need for fungicides with low environmental impact and no effect on non-target organisms. To this aim we are isolating new molecules able to counteract $P$. viticola effectors identified during transcriptome analysis by avoiding their interaction with the plant immune-system. In conclusion, an important source of resistance to P. viticola, that could be exploited in traditional breeding techniques or genome editing was discovered. Novel potential fungicides are under investigation. These tools can contribute individually to the management of downy mildew, but show their greatest potential if used synergistically.

This work has been supported by University of Milan, DiSAA, Research Support Plan 2015-2017 "Genome editing e sviluppo di fungicidi a basso impatto ambientale: una strategia sinergica per la difesa sostenibile dalla peronospora della vite".

\section{Studying proteomic changes in Volatile Organic Compounds (VOCs) primed plants}

\author{
I. Mancini ${ }^{1}$, M.M. Monti ${ }^{2}$, L. Gualtieri ${ }^{2}$, M. Marsoni ${ }^{1}$, C. Vannini ${ }^{1}$, F. \\ Loreto $^{2}$, M. Bracale ${ }^{1}$, M. Ruocco ${ }^{2}$
}

${ }^{1}$ Università degli studi dell'Insubria, Via J.H. Dunant 3, 21100, Varese (VA); ${ }^{2}$ Istituto per la Protezione Sostenibile delle Piante (IPSP), Via Università, 133-80055,Portici (NA).Email: michelina.ruocco@ipsp.cnrit

Plants during their life emit species and cultivar specific Volatile Organic Compounds (VOCs) blends. Environment strongly influences plant development, consequently modulating VOCs emission, both in terms of quantity and quality. From recent studies it is known that plants use VOCs to communicate not only among them, but also with their antagonists and symbionts. The final aim of this work is the study of Phaseulus vulgaris / Colletotrichum lindemuthianum interaction as a model system, investigating, at proteomic level, on the priming effect of VOCs emitted by C. lindemuthianum tolerant bean plants on neighbouring susceptible plants. VOCs of two different $P$. vulgaris cultivars, a resistant (R) and a susceptible (S) one, have been analysed with PTR-MS TOF, a sophisticated in vivo real time detection system. The two different cultivars presented a specific VOCs emission profile, which changed after pathogen infection. Indeed, VOCs emitted by R and S lines, when compared, differed both in terms of quantity (with a modulation of some compounds) and of quality (ex-novo VOCs release) when infection occurred.

We are now analysing the proteomic changes, occurred in $\mathrm{S}$ uninfected plants after receiving VOCs from $\mathrm{R}$ infected plants. We believe that our findings will pave the way for the development of more sustainable crop protection strategies based on plant-VOCs induced priming offering a cheaper, effective, ecofriendly alternative to synthetic pesticides and fertilizers. This work was supported by accordo Bilaterale CNR-CONACYT 2017-18

\section{Resistance inducers may influence leaves endophytic mi- croflora}

F. Margheri ${ }^{1}$, G. Carella ${ }^{1}$, A. Pacetti ${ }^{1}$, L. Mugnai ${ }^{1}$, A. Fabiani ${ }^{2}$, S. Mocali $^{2}$

${ }^{I}$ Department of Agricultural, Food, Environmental and Forestry Science and Technology (DAGRI), Plant pathology and Entomology section, University of Florence, P.le delle Cascine, 28, 50144 Firenze, Italy; ${ }^{2}$ Council for Agricultural Research and Economics (CREA), Research centre for Agriculture and Environment, Via di Lanciola, 12/A, 50125 - Cascine del Riccio (Firenze, Italy).E-mail: stefano.mocali@crea.gov.it

Different strategies for grapevine protection (organic and integrated protection) were compared in experimental trials conducted on Vitis vinifera $\mathrm{cv}$. Sangiovese in a production vineyard (Chianti Classico), to evaluate the effectiveness of agronomic techniques and foliar applications based on the use of resistance inducers, to support control of Plasmopara viticola on grapevines. Microorganisms can establish 
different types of interactions with superior organisms, in some cases very narrow or obligatory. Microbial communities associated with plants are defined as the "plant microbiome" and it is extensively demonstrated that plants host in their internal tissues a set of fungi and bacteria, called endophytes, which may play a beneficial role on the host fitness under biotic and/or abiotic stress. Therefore, a part of this work is focused on the quantitative and qualitative assessment of the cultivable endophytic bacterial communities present in the leaves of plants affected by downy mildew, focusing attention on the treatments with organic management. The microbiological analysis revealed endophytic bacteria belonging to at least three different haplotypes that were identified by sequencing the $16 \mathrm{~S}$ rDNA gene. The dominant haplotype $(\approx 99 \%)$ was Bacillus amyloliquefaciens, which is known for its biocontrol properties. The results showed that the abundance of B. amyloliquefaciens in the samples, taken in every treatment, had an increasing trend as did the quantities of the resistance inducers applied, suggesting a potential role of these products in inducing the selection of beneficial endophytes by the plant.

This work was included in the framework of the project "LIFE GREEN GRAPES, New approaches for protection in a modern sustainable viticulture: from nursery to harvesting".

\section{Windblow, One (Plant) Health on stage. Arising Research through the theatre}

\section{A. Masino ${ }^{1}$, I. Ferri ${ }^{2}$, L. Vallarino Gancia ${ }^{3}$, M.L. Gullino ${ }^{1,4}$ \\ ${ }^{1}$ Università degli Studi di Torino, Centro AGROINNOVA, Largo P. Braccini 2 - 10095 Grugliasco (TO), Italy; ${ }^{2}$ Teatro Tangram di Torino, Via Don Luigi Orione 5 - 10141 Torino (TO); ${ }^{3}$ Teatro Stabile di Torino - Teatro Nazionale, Via G. Rossini 12 - 10124 Torino (TO); ${ }^{4}$ Università degli Studi di Torino, DISAFA, Largo P. Braccini 2 - 10095 Grugliasco (TO), Italy.E-mail: andrea.masino@unito.it}

Dissemination and communication are relevant for European research projects. Especially for multi-actor approach project focusing research activities on the needs of the stakeholders, research is looking for new ways of engaging with civil society and policy makers. Theatre is considered a place to go in order to get points of reflection and thoughts; the show ensures the effective and efficient spread of the scientific results to a wide public.

In 2018, Agroinnova co-produced 'Windblow', a show experience focused on the One Health approach. The show went on the stage of the Carignano Theatre in Torino on October $15^{\text {th }}$ (World Food Day) and replied in the frame of an important Summer Festival (2019) in Exilles. Artists performed to sensitize the audience (one thousand attendees) about the role of plant health in environment and landscape protection. By the deepest meaning of environmental thoughts, Windblow is a short trip, where Science and Poetry are shown us as guides. By the direction of Ivana Ferri and through the participation of Massimo Germini, Bruno Maria Ferraro, Celeste Gugliandolo and Patrizia Pozzi, theatre and music mixed together in a perfect recipe.

The collaboration with several partners enabled to build a new stage representation on the importance of plants and their health in the frame of global health. Such performance will be able to reach new different locations, arising the role of Plant Health in our lives.

The project has received funding from the European Union Horizon 2020 research and innovation program under grant agreement number 634179 "Effective Management of Pests and Harmful Alien Species-Integrated Solutions" (EMPHASIS) and under grant agreement number 633999 "EU-China Lever for IPM Demonstration” (EUCLID).

\section{The International Year of Plant Health in Piedmont: a call-to-action for 2020}

\author{
A. Masino ${ }^{1}$, M.L. Gullino ${ }^{1,2}$, R. Lopian ${ }^{3}$ \\ ${ }^{1}$ Università degli Studi di Torino, Centro AGROINNOVA, Largo P. \\ Braccini 2 - 10095 Grugliasco (TO), Italy; ${ }^{2}$ Università degli Studi di \\ Torino, DISAFA, Largo P. Braccini 2 - 10095 Grugliasco (TO), Italy; \\ ${ }^{3}$ Ministry of Agriculture and Forestry of Finland, PO Box 30, FI-00023 \\ Government. E-mail: andrea.masino@unito.it
}

On 20 December 2018 The UN Food and Agriculture Organization and the International Plant Protection Convention Secretariat, based at FAO, welcomed the UN General Assembly's adoption of a resolution proclaiming 2020 as the International Year of Plant Health (IYPH). IYPH will be joined globally by all plant protection's actors to raise awareness about the role of plant health for our lives and the importance to protect the world's plant resources from pests and diseases.

Agroinnova, the Centre of Competence for the innovation in the agroenvironmental field of the University of Torino, in cooperation with local institutions, will support and promote a multitude of activities with the aim of positioning Torino and Piedmont as active actors of the IYPH by 2020. Our vision: a "road map" of initiatives linked to each other by the theme of plant health, leading to a major event, a cultural festival, to be held in Torino in 2020 from June 4 to 6.

As the dissemination of scientific results affects society as a whole, it is crucial that citizens better understand how plant health impacts sustainable agriculture and food security, as well as ecosystem protection. Artistic performances, concerts, exhibitions, shows, talks, round tables, workshops, open days and several cultural formats are able to communicate the results of research more effectively. Communication allows to start new projects, create networks of excellence to share experiences, involve more stakeholders and get support for new projects.

The project has received the patronage of Regione Piemonte and the International Plant Protection Convention. Special thanks to Antonella Parigi, Giorgio Ferrero and Alberto Valmaggia, former Regione Piemonte's Councillors, and to Mirko Montuori, IPPC Governance and Public Information Specialist, for their support.

New diseases on leafy vegetable and ornamental plants in
Italy caused by Paramyrothecium and Albifimbria species

\section{S. Matić ${ }^{1}$, G. Gilardi ${ }^{1}$, M.L. Gullino ${ }^{1,2}$, A. Garibaldi ${ }^{1}$}

${ }^{1}$ Centre of Competence for the Innovation in the Agro-environmental Sector (AGROINNOVA), University of Turin, Largo Braccini 2, 10095 Grugliasco (TO), Italy; ${ }^{2}$ Department of Agricultural, Forest and Food Sciences (DISAFA), University of Turin, Largo Braccini 2, 10095 Grugliasco (TO), Italy.E-mail: slavica.matic@unito.it

Severe leaf spot and plant decline of leafy vegetables caused by Myrothecium roridum and Myrothecium verrucaria have recently been described in northern and southern Italy. The Paramyrothecium and Albifimbria genera have lately been separated from the genus Myrothecium, comprising two phytopathogenic fungi, Paramyrothecium roridum (the former M. roridum) and Albifimbria verrucaria (the former $M$. verrucaria), and other soil-inhabiting fungal species. Thirty-nine strains isolated from affected plants of various leafy vegetables and ornamental species were selected for 
morphological and molecular characterization. Morphological observations assigned 21 strains to Albifimbria verrucaria, and 18 strains to Paramyrothecium-like fungi (without precise species identification). Six-loci molecular characterization allowed the assignment of all strains into four species: A. verrucaria, $P$. roridum, $P$. foliicola and $P$. nigrum. Concatenated phylogenetic analyses grouped the isolated fungi into four distinct clusters. Albifimbria verrucaria, Paramyrothecium roridum, $P$. foliicola, and P. nigrum were able to induce necrotic leaf spots as single species in pathogenicity assays, confirming to be the causal agents of the leaf spot disease. The involvement of previously described saprophytic fungi, $P$. foliicola and $P$. nigrum, in plant diseases for the first time is of considerable importance, requiring further studies for their efficient management. Furthermore, some of Paramyrothecium and Albifimbria species are known to be seed-transmitted, which increases the risk of their introduction and spread into new geographic areas. More molecular and epidemiological studies are needed to elucidate inter- and intraspecies evolutionary aspects of these fungi, the members of the polyphyletic Stachybotriaceae family.

The research that has led to these results has received funding from the European Union's Horizon 2020 research and innovation programme under grant agreement number 633999 "EU-CHINA Lever for IPM Demonstration" (EUCLID).

\section{Role and importance of accredited private laboratories for the phytosanitary surveillance in Italy: challenges and ad- vantages}

\section{Mezzalama, D. Spadaro, S. Matic, G. Gilardi, A. Garibaldi, M.L. Gullino}

AGROINNOVA, University of Torino, Italy, Largo Paolo Braccini 2, 10095 Grugliasco, Italy.E-mail: monica.mezzalama@unito.it

In the frame of the Italian regulation no. 214 of August 19, 2005, in line with the EU 2002/89/CE, the National Plant Protection Organization (NPPO) can accredit private diagnostic laboratories to carry out plant testing with official recognition. The laboratory of the Centre of competence AGROINNOVA of the University of Torino, Italy, is accredited by the regional NPPO for the detection of fungal pathogens in crops of economic importance in Italy (vegetables, ornamentals, grapevine, fruit, cereals). The laboratory has recently engaged in the European Project Horizon 2020 VALITEST for the validation of diagnostic tests to support plant health and surveillance. So far, the laboratory carried out molecular and serological Test Performance Studies (TPS) organized by two different European reference laboratories to detect Erwinia amylovora with three PCR, one LAMP and two serological protocols; Pantoea stewartii with six PCR protocols, and Fusarium circinatum with five PCR protocols. TPS constitute a challenge for a laboratory where technical and scientific competence are compared at an international level and the results are the passport to widen the scope of the accreditation of the laboratory. The role of private reference laboratories is primary in a country like Italy, where the NPPO is fragmented in the regional administrations with different levels of efficiency. One of the most successful strategy to prevent the outbreak of dangerous plant diseases is the continuous surveillance that requires: resources, efficient communication, effective national and international networks between the academy, that can provide the updated scientific competence, and the NPPO.

Work supported by EU grant agreement no. 773139.
Effect of onion yellow dwarf virus infection on 'Rossa di Tropea' onion bulb quality and organoleptic properties

\author{
G. Micali, A. Tiberini, F. Araniti, A. Dattola, A. Piscopo, G. Gullo, G. \\ Albanese
}

Università degli Studi Mediterranea di Reggio Calabria, Dipartimento di AGRARIA, Località Feo di Vito - 89122 Reggio Calabria, Italy. E-mail: giuseppe.micali@unirc.it

Onion yellow dwarf virus (OYDV, genus Potyvirus) has been reported to be the most widely spread virus in onion and Allium spp., causing detrimental effects on crop and bulb yield. Despite the agronomic effects of the viral infection in onion are well documented, little information is available about the effect of OYDV on bulb organoleptic properties and quality. "Rossa di Tropea" onion, a particular onion cultivated in Calabria region (Italy), with a Protected Geographical Indication (PGI) trademarks is characterised by a high nutraceutical compounds content. In this study, the effect of OYDV infection on overall bulb quality of "Rossa di Tropea" onion was evaluated, determining the total volatile organic compounds (VOCs) in healthy and infected bulbs by gas chromatography-mass spectrometry (GC-MS), highlighting a structural alteration and modulation of compounds related to flavor and organoleptic properties in the infected bulbs. In addition, the total content of ascorbic acid (AA-Vitamin C) was determined and a double AA degradation rate was observed in infected bulbs. Moreover, a sensory analysis was performed by 10 panelists, addressed to evaluate general and specific attributes of acceptability of healthy/infected bulbs (i.e. odor, color, pungency, spiciness, etc.). In general, it was observed that OYDV could reduce the bulb sweetness and crunchiness, increasing spiciness and pungency, altering overall organoleptic features of "Rossa di Tropea".

This study was carried out as part of planned activities of the project "SI.ORTO" funded by Italian Ministry of Education, to evaluate OYDV infection effect on modulation and variation of onion secondary metabolites.

\section{The effectiveness of different storage methods for the shelf-life and the fungal populations of the Hungarian tart cherry varieties}

\author{
K. Mihály ${ }^{1}$, F. Takács ${ }^{2}$, E. Sándor ${ }^{1}$ \\ ${ }^{1}$ University of Debrecen, Faculty of Agricultural and Food Sciences and \\ Environmental Management, Food Science Institute, 4032 Debrecen, \\ Böszörményi st, 138, Hungary; ${ }^{2}$ National Agricultural Research and \\ Innovation Centre, Fruit Research Institute, 4244 Újfehértó, Vadastag \\ 2, Hungary.E-mail:mihaly.kata@agr.unideb.hu
}

Hungary is among the three largest producers in the European Union, also the world's largest fresh tart cherry exporter. In case of cherry (Prunus avium L.) and tart cherry (Prunus cerasus L.), half of the loss was estimated to be caused by moulds. There are restricted information about the effectivity for decreasing postharvest losses, primarily caused by postharvest diseases. The optimization of the storage and the shelf-life of the fruits could provide longer time for availability of fresh fruit with high nutritional value. The aim of our research was the investigation of the normal and the modified atmosphere packaging (MAP) during cold storage following different preharvest treatments on three Hungarian sour cherry varieties: 'Érdi bőtermő', 'Petri', and 'Újfehértói fürtös'. The surface mould number and the morphology based identification of moulds were detected following harvest. Moulds were isolated from rotten fruits during shelf-life studies and identified based on morphological and 
molecular characters. Disease incidence (DI) was also detected at $20^{\circ} \mathrm{C}$. These tests were repeated after six weeks storage at $0-2^{\circ} \mathrm{C}$, in normal atmosphere and MAP. It was concluded that MAP storage decreased the decay percentage. In the shelf-life test, the incidence of decay was significantly higher after storage than after harvesting. Fruit surface mould number changed after storage: storage increased the CFU number of sour-cherry, but in the case of MAP storage, this value was lower. The work/publication is supported by the EFOP-3.6.3-VEKOP-16-201700008 project. The project is co-financed by the European Union and the European Social Fund.

\section{The $\mathrm{Na}^{+} / \mathrm{Ca}^{2+}$ exchanger is critical for virulence of Pseudomonas savastanoi pv. savastanoi and Pseudomonas syringae pv. tomato}

\section{Moretti $^{1}$, S. Trabalza ${ }^{1}$, E. Caballo-Ponce ${ }^{2}$, C. Ramos $^{2}$, H.A. van den Burg ${ }^{3}$, C.A. Palmerini ${ }^{1}$, R. Buonaurio ${ }^{1}$}

${ }^{I}$ Department of Agricultural, Food and Environmental Science (DSA3) University of Perugia, Borgo XX Giugno 74, 06121 Perugia, Italy; ${ }^{2}$ Instituto de Hortofruticultura Subtropical y Mediterránea La Mayora, Universidad de Málaga-Consejo Superior de Investigaciones Científicas (IHSM-UMACSIC), Área de Genética, Málaga, Spain, ${ }^{3}$ Molecular Plant Pathology, Swammerdam Institute for Life Sciences (SILS), University of Amsterdam, The Netherlands.E-mail: chiaraluce.moretti@unipg.it

$\mathrm{Ca}^{2+}$ represents an important environmental clue, as reported for bacteria infecting mammalians. We, here, demonstrate that $\mathrm{Ca}^{2+}$ entry in Pseudomonas savastanoi pv. savastanoi (Psav) strain DAPP-PG 722 and Pseudomonas syringae pv. tomato (Pst) strain DC3000 is mediated by a Na${ }^{+} / \mathrm{Ca}^{2+}$ exchanger critical for virulence. Using the fluorescent $\mathrm{Ca}^{2+}$ probe Fura 2-AM, we demonstrate that $\mathrm{Ca}^{2+}$ enters Psav and Pst cells foremost when they experience low levels of energy, a situation mimicking the apoplastic fluid. In fact, $\mathrm{Ca}^{2+}$ entry was suppressed in the presence of high concentrations of glucose, fructose, sucrose or ATP. Since $\mathrm{Ca}^{2+}$ entry was inhibited by nifedipine and $\mathrm{LiCl}$, we conclude that the channel for $\mathrm{Ca}^{2+}$ entry is a $\mathrm{Na}^{+} / \mathrm{Ca}^{2+}$ exchanger. In silico analysis of the Psav DAPP-PG 722 and Pst DC3000 genomes revealed the presence of a single gene coding for a $\mathrm{Na}^{+} / \mathrm{Ca}^{2+}$ exchanger (cneA), which is a widely conserved and ancestral gene within the P. syringae complex based on gene phylogeny. Mutation of $c n e A$ inhibited the hypersensitive response in tobacco leaves and disease symptom development in olive and tomato plants. In Psav-cneA mutant, the expression of both pathogenicity ( $h r p L$, $h r p A$ and $i a a M$ ) and virulence ( $p t z$ ) genes was reduced. Complementation of the Psav-cneA mutation restored both $\mathrm{Ca}^{2+}$ entry and pathogenicity in olive plants, but failed to restore the HR in tobacco leaves. In conclusion, $\mathrm{Ca}^{2+}$ entry acts as a 'host signal' that allows and promotes Psav and Pst pathogenicity.

This work was financially supported by DSA3 research funds "Fondo di base".

\section{Enzymatic and non-enzymatic wood-decay pathway char- acterization in Fomitiporia mediterranea}

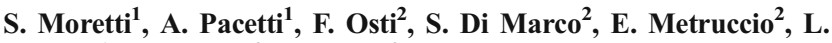

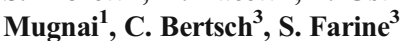

${ }^{1}$ Department of Agricultural, Food, Environmental and Forestry Science and Technology (DAGRI), Plant pathology and Entomology section, University of Florence, P.le delle Cascine, 28, 50144 Firenze, Italy; ${ }^{2} C N R$ IBE, Via Gobetti 101, 40129 Bologna, Italy; ${ }^{3}$ Laboratoire Vigne Biotechnologies et
Environnement UR-3991, Université de Haute Alsace, 33 rue de Herrlisheim, 68000 Colmar,France.E-mail:samuele.moretti@unifi.it

Among grapevine trunk diseases-related fungi, the basidiomycete Fomitiporia mediterranea (Fmed) is responsible for wood-decay in Vitis vinifera, showing an intermediate behaviour between brownand white-rot fungi. Nowadays, it is widely accepted that, in brown-rot fungi, wood decay is the result of synergic enzymatic and non-enzymatic degrading action. In the first one, enzymatic action, laccases and manganese peroxidases $(\mathrm{MnP})$ play a major role in catalysing lignin degradation, splitting $\mathrm{C}_{\alpha}-\mathrm{C}_{\beta}, \beta$-aryl, $\mathrm{C}_{1}-\mathrm{C}_{\alpha}$ and aromatic $\mathrm{C}-\mathrm{C}$ bonds. In the second one, non- enzymatic action, the lignin/cellulose degradation is attributed to the non-enzymatic Fenton/Haber Weiss pathway, generating hydroxyl radicals within the wood cell, also thanks to Fe oxidation. Because low-molecularweight (LMW) iron-binding compounds mediate this wood degradation, the mechanism has been named "chelator-mediated Fenton" (CMF) reaction. Since it is still not clear if Fmed has both mechanisms of lignin/cellulose degradation (enzymatic and CMF reactions), these two pathways were evaluated screening 13 Fmed strains, assaying: i) laccase and $\mathrm{MnP}$ activity; ii) the strains capability to produce LMW iron-binding compounds, ferrous-iron $\left(\mathrm{Fe}^{2+}\right)$ accumulation and the consequent production of hydroxyl radicals.

\section{Differentiation of Melampsoridium species causing alder rust by DNA sequence data and morphology}

\section{S. Moricca $^{1}$, C. M. O. Longa ${ }^{2}$, A. Benigno ${ }^{1}$, G. Maresi $^{3}$}

${ }^{1}$ Department of Agricultural, Food, Environmental and Forestry Science and Technology (DAGRI), Plant Pathology and Entomology Division, University of Florence, Piazzale delle Cascine 28, 50144, Firenze, Italy; ${ }^{2}$ Centre for Technology Transfer, Fondazione Edmund Mach Via E. Mach 1, 38010 San Michele all'Adige (TN), Italy; ${ }^{3}$ Department of Sustainable Agroecosystems and Bioresources, Research and Innovation Centre, Fondazione Edmund Mach. Via E. Mach 1, I-38010, San Michele all'Adige, Trento, Italy.E-mail: salvatore.moricca@unifi.it

Three rust fungi, Melampsoridium alni, M. betulinum and M. hiratsukanum (Pucciniastraceae, Pucciniales) have been reported as pathogens of Alnus sp. in Europe. These foliar pathogens cause in particularly wet and rainy years severe defoliation to various Alnus species. In some valleys of eastern Italian Alps, rust outbreaks have been occurring for about a decade on grey alder (Alnus incana), plaguing riparian formations of this species. Recently, a re-examination of older records of rusts on European alders and of herbarium specimens led to the hypothesis that some of the previous reports may be incorrect. In light of these doubts, a microscopic, ultrastructural and molecular investigation was conducted to ascertain the identity of the rust fungus that is occurring on grey alder in the Italian Alps. Optical and SEM analyses of the morphology of uredinia (ontogeny, geometry of peridial cells, length of ostiolar cells) and urediniospores (shape, roundness, dimensions, echinulation, germ pore number and distribution) showed that the fungus found on grey alder is $M$. hiratsukanum. A comparison of the ITS-rDNA region from $M$. hiratsukanum, with those from M. alni and M. betulinum deposited in the GenBank database revealed a sequence homology of $98.4 \%$ and of $97.77 \%$, respectively. A CLUSTAL alignment showed a considerable variation in the two internal transcribed spacers ITS1 and ITS2 between $M$. hiratsukanum and the other two fungi, substantiating in both point mutations and length polymorphism. This study has definitively confirmed that the rust fungus that infects grey alder in some valleys of Trentino-Alto Adige is M. hiratsukanum. 


\section{Sanitary status of chestnut orchards in Marche region}

\author{
S. Murolo ${ }^{1}$, A. Santini ${ }^{2}$, A. Corvaro ${ }^{1}$, V. Gasperini ${ }^{1}$, G. Romanazzi ${ }^{1}$ \\ ${ }^{1}$ Department of Agricultural, Food and Environmental Sciences, Marche \\ Polytechnic University, Via Brecce Bianche, 60131 Ancona, Italy; \\ ${ }^{2}$ Comunanza Agraria Monteacuto, Acquasanta Terme (AP), Italy. E- \\ mail:s.murolo@univpm.it
}

Sweet chestnut (Castanea sativa) is widespread in Europe and it is an important multipurpose tree in the Mediterranean area, as it is used for its wood, fruit, honey and tannin. From 2016 up 2018, surveys were carried out in order to determine the phytosanitary status of chestnut orchards in Marche region. In the surveyed areas (geographical coordinates 42 44'16" $80 ; 13$ 24'29"16), a low-medium percentage of plants affected by chestnut blight, caused by Cryphonectria parasitica both virulent and hypovirulent strains, was found. A recrudescence of chestnut ink disease was assessed both for young and even more for ancient and monumental plants along the "Sentiero dei Patriarchi", located in Umito (Acquasanta Terme, AP, Italy). We also recorded a disease, reported since 1992-93 and called "ricciarella" from the local chestnut growers, whose aetiology is still unknown. The main symptoms were the discoloration of interveinal tissues, distortion and marginal necrosis of leaf blame, abortion of fruit, and plant decline. The symptoms appeared at the beginning of June and increased in severity during the season. In Marche region, considering the economic importance of chestnut and the decline of young and old plants which occurred and increased in number of plants year by year, "ricciarella" could be considered an emerging disease. Biomolecular analyses are in progress to better understand the aetiology of the unknown disease and to plan potential management strategies.

Characterization of the promoter sequence of Rapid Alkalinization Factor (RALF) gene in Fragaria $x$ ananassa interacting with Colletotrichum acutatum

\section{F. Negrini ${ }^{1}$, K. Ogrady ${ }^{2}$, K.M. Folta ${ }^{2}$, E. Baraldi ${ }^{1}$ \\ ${ }^{1}$ University of Bologna, DISTAL (Department of Agricultural and food Science), Viale Fanin 46, 40127, Bologna, Italy; ${ }^{2}$ Horticultural Sciences Department, University of Florida,1301 Fifield Hall, Gainesville, FL 32611,USA.E-mail: elena.baraldi@unibo.it}

Rapid alkalinization factor $(R A L F)$ genes are ubiquitous in plant kingdom and encode for small signal peptides able to trigger a rapid increase of apoplastic $\mathrm{pH}$ trough the interaction with Malektin-Like receptor kinase, FERONIA. RALF genes are involved in fertilization, cell elongation, root growth, and recently, they have been characterized as suppressors of plant immunity response. Furthermore, $R A L F$ homologs were found in the genome of many plant fungal pathogens, resulting to be crucial for their infection ability. Transcriptional analysis conducted on strawberry (Fragaria x ananassa) infected with Colletotrichum acutatum revealed an increased expression of a $R A L F$ gene in the susceptible red fruits. This finding is consistent with previous evidence from plant-fungi interaction transcriptome analysis, supporting the hypothetical involvement of $R A L F$ as immunity response regulator. Fragaria $\mathrm{x}$ ananassa $R A L F$ genes were identified and a heatmap expression analysis was done comparing different tissues, based on published RNA-seq data. Then, different RALF homologs expression was tested by qRT-PCR on red and white fruits after $C$. acutatum and $B$. cinerea infection. In order to understand the mechanism behind the $R A L F$ response to pathogen, the 5' upstream sequence of strawberry $R A L F$ 33-like gene was analyzed using MotifLab software and PLACE database to predict putative cis-acting and transcription factor binding motifs. Progressive truncated fragments of RALF 5' upstream sequences were cloned into GUS:eGFP reporters and contructs were agroinfilitrated in fruits. From qRT PCR and GUS histochemical analysis of transient transformed and infected fruits it was not possible to identify a specific region responsible for pathogen response and further analysis of other regions flanking $R A L F$ gene in strawberry will be necessary in order to precisely identify the inducible promoter elements.

\section{Study of the olive anthracnose within the Italian national project "SALVAOLIVI"}

\section{Orzali, M.T. Valente, G. Di Giambattista, L. Riccioni}

CREA-DC, Research Centre for Plant Protection and Certification. Email: luca.riccioni@crea.gov.it

The SALVAOLIVI project is part of the Intervention Plan for the Italian olive/oil sector and has the general objective of safeguarding and enhancing the Italian olive-growing heritage through research actions in the field of phytosanitary defence. Within this project, the main aim of the WP 2.2.2 is to define, in the various Italian cultural environments, which fungal species are involved in olive anthracnose and which are the optimal environmental conditions for their diffusion, to provide useful information to be used for disease control. The anthracnose of the olives is caused by different species of Colletotrichum and determines massive damages (decrease in olive fruit production and oil quality), depending on the environmental conditions, the susceptibility of the olive cultivar and the virulence of the pathogen's population.

The performed activity, subdivided into 2 Tasks, consisted in monitoring and sampling drupes with rot symptoms from 15 places in Lazio, Umbria and Sardinia and collecting the associated fungi. The populations of the collected Colletotrichum species were studied, together with their genetic variability (ITS and beta-tubulin regions sequencing) and variability in aggressiveness (fruits artificial inoculation). In the $2^{\text {nd }}$ task, the monitoring of inoculation dispersion in the air has been performed with a volumetric spore trap (Burkard Manufacturing Co Ltd) located in Lazio, and molecular protocols for Colletotrichum spp. detection from the spore trap tape were set up, in order to quantify the spore dispersion during the fruit development. The next years activity will consist in analogous surveys and sampling of drupes in Calabria and Sicily and in a creation of a predictive model for infection based on dissemination of Colletotrichum conidia, plant vegetative stage and climatic trend.

The present project is founded by MIPAAFT

First scientific attempt to evaluate the efficacy of trunk surgery on grapevine as a tool to suppress GLSD symptoms: a comparison between mechanical and chemical approach

\section{A. Pacetti ${ }^{1}$, S. Moretti ${ }^{1}$, S. Farine ${ }^{2}$, C. Bertsch ${ }^{2}$, L. Mugnai ${ }^{1}$}

${ }^{1}$ Department of Agricultural, Food, Environmental and Forestry Science and Technology (DAGRI), Plant pathology and Entomology section, University of Florence, P.le delle Cascine, 28, 50144 Firenze, Italy; ${ }^{2}$ Laboratoire Vigne Biotechnologies et Environnement UR-3991, Université de Haute Alsace, 33 rue de Herrlisheim, 68000 Colmar, France.E-mail: andrea.pacetti@unifi.it

Esca complex is currently regarded as one of the most devastating grapevine trunk diseases (GTDs) in European vineyards. Sodium arsenite has been considered to be the only effective pesticide used in some countries to control the foliar symptom expression of Esca, but its use is now 
banned in Europe. Current disease control strategies do not include techniques to treat diseased plants in the field but only to limit and minimize the number of new infections by protection of the pruning wounds in the field and optimization of hygiene of the equipment and plant material in the nursery. A recent practice reported to decrease the number of symptomatic plants is trunk surgery or "curetage du bois" also known as "dendrochirurgia". Besides this, plant chemical endotherapy applications may offer a practical strategy for GTDs managements. Nowadays, those two approaches are sometimes used or tested in fruit crop production and also in some forest trees for protection to control pathogens, but their mechanism of action is not well documented. The mechanical approach was tested in the vineyard where three levels of trunk surgery were applied: $i$ ) a cut made by chainsaw from one side to the other; $i$ i) a half decay removal; iii) a complete decay removal. The endotherapic chemical approach was applied by drilling the vines from the top, and filling the hole with different chemical solutions. Studies are performed to investigate on the changes induced by the treatment at different levels.

\section{Phytotoxic metabolites produced by Diaporthe eres, one of the causal agents of cane bleaching of grapevine in Italy}

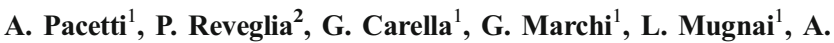 \\ Evidente $^{2}$
}

${ }^{1}$ Department of Agricultural, Food, Environmental and Forestry Science and Technology (DAGRI), Plant pathology and Entomology section, University of Florence, P.le delle Cascine, 28, 50144 Firenze, Italy; ${ }^{2}$ Department of Chemical Sciences Complesso Universitario Montesant'Angelo, University of Naples Federico II, Via Cintia 4, 80126, Naples, Italy.E-mail: andrea.pacetti@unifi.it

Phomopsis cane and leaf spot is known to be caused by Diaporthe ampelina, causing the abortion of buds and the appearance of necrotic patches at the base of internodes, which can reach the cortical tissues and cause cracks. Over time, the plants weaken and spurs or cordons necrotize. Among other Diaporthe species associated to grapevine, $D$. eres was reported to be associated with bleaching of the affected canes with black pycnidia emerging from the plant bark. Diaporthe species are also known to produce phytotoxic metabolites the role of which in the disease was not clarified. In order to evaluate whether $D$. eres is also able to produce metabolites that may be involved in the development of symptoms, the ability of strain M149 to produce phytotoxic metabolites was investigated. Five potentially toxic compounds were identified from liquid cultures by their physical and spectroscopic properties, namely tyrosol, $p$-cresol, 4-hydroxybenzaldehyde, $p$ hydroxybenzoic acid and nectriapyrone. All of them, showed phytotoxic effects of different intensity when tested at 0.5 and $1.0 \mathrm{mg} / \mathrm{ml}$ on the host plant tissues causing an intense reddening of the grapevine leaf veins, petiole necrosis, a progressive brown discolouration and necrosis on grapevine leaf disk assays. On tomato, a non-host species, only damage to the petiole of the youngest leaf was recorded after $24 \mathrm{~h}$. The role of the phytotoxic metabolites of $D$. eres in symptom development needs to be investigated and clarified.

\section{Evaluation of the effect of bacteria isolated from the ectomycorrhizosphere on Tuber borchii growth in vitro}

\section{S. Pagliarani, A. Vannini}

Department for Innovation in Biological, Agro-food and Forest systems (DIBAF), University of Tuscia, Via S. Camillo de Lellis, snc, 01100 ViterboVT.E-mail:pagliarani@unitus.it
The ectomycorrhizal symbiosis is a mutualistic association between the mycorrhizal fungus and the roots of host plants that occurs in soils and forests all over the world. In this study, C. avellana plants mycorrhized with Tuber aestivum were selected from a truffle ground located in the municipality of Sutri (Viterbo). The ectomycorrhizal root tips of the plants were selected for the isolation of culturable mycorrhiza-associated bacteria. After growth on selective medium, DNA was extracted from the colonies for amplification of the full-length 16S rDNA. After sequencing and molecular characterization, the isolates were utilized for the in vitro assay on T. borchii mycelial growth. From the sequencing of the bacterlal isolates, five were identified as strains belonging to the Pseudomonas and Bacillus genera, confirming that these groups of bacteria represent a considerable fraction of the total population residing in the mycorrhizae of the genus Tuber. After 15 days of dual growth, most isolates inhibited fungal growth compared to the control, except the bacterial strain identified as Bacillus megaterium, which promotes the growth of $T$. borchii by $5 \%$ (differences are statistically significant, ANOVA, $\mathrm{F}=$ 271.9, $\mathrm{P}<0.001$ ). These results suggest the putative growth-stimulating activity of spore-forming strains, in agreement with studies showing that the bacteria isolated from the mycorrhizosphere are able to enhance fungal growth and subsequent establishment of mycorrhizas. Further in vitro investigations are needed to asses whether the bacteria identified in this study can be used for biotechnological application.

Deciphering the role of the on-farm compost microbiome in natural soil-borne disease suppressiveness by highthroughput metagenomic analysis

\section{Pane ${ }^{1}$, R. Scotti ${ }^{1}$, R. Sorrentino ${ }^{2}$, M. Molisso ${ }^{3}$, A. Di Matteo ${ }^{3}$, M. Zaccardelli $^{1}$}

${ }^{1}$ Consiglio per la Ricerca in Agricoltura e l'Analisi dell'Economia Agraria, Centro di Ricerca Orticoltura e Florovivaismo, via Cavalleggeri, 25 - 84098 Pontecagnano Faiano (Salerno); ${ }^{2}$ Consiglio per la Ricerca in Agricoltura e l'Analisi dell'Economia Agraria, Centro di Ricerca Cerealicoltura e Colture Industriali (Laboratorio di Caserta), via Torrino, 2 - 81100 Caserta, Italy; ${ }^{3}$ Università degli Studi di Napoli Federico II, Dipartimento di Agraria, via Università 100-80055 Portici (Napoli).E-mail: catello.pane@crea.govit

Compost disease suppressiveness mainly relies on biological control mechanisms of the resident microbes. Here, a set of on-farm composts was evaluated for suppressiveness against the soil-borne pathogens Rhizoctonia solani and Sclerotinia minor on Lepidium sativum. A differential response of the samples in controlling the target diseases was highlighted. The net contribution of the biological component was measured by comparing control levels in raw and autoclaved composts and related to the biodiversity indices computed by the t-RLFP analysis of 16S rRNA gene amplicons. Communities of two composts, selected for the high and low suppressiveness, were subsequently characterized by metagenomic analysis performed on $16 \mathrm{~S}$ and $18 \mathrm{~S}$ rRNA gene partial sequences and deepened with a whole genome shotgun (WGS) sequencing. 16S amplicon sequences for the 10 most abundant phyla, covering $95 \%$ of annotation, outlined very high abundances of Proteobacteria, Bacteroidetes, Actinobacteria, Planctomycetes and DeinococcusThermus, all of them were most abundant in the suppressive compost, except the Planctomycetes phylum that was most abundant in the less suppressive. 18S amplicon showed lower diversity with respect to $16 \mathrm{~S}$ portion at the phylum level, covering less than $60 \%$ of annotation and showing only two main phyla, Basidiomycota most abundant in the suppressive compost, and Ascomycota most abundant in the other one. At genus level, Galbibacter, Truepera and Luteimonas are significantly increased in the more suppressive one, on the other hand Flavobacterium and Streptomyces genera were higher in the less suppressive one. The 
depth of the WGS sequencing confirmed most of the results reported with the amplicon sequencing.

This work was supported by EU LIFE+ program, LIFE12ENV/IT/ 000719, CarbOnFarm project

\section{Reverse transcribed quantitative polymerase chain reac- tion for rapid screening of tomato brown rugose fruit virus}

\section{S. Panno ${ }^{1}$, A.G. Caruso ${ }^{1}$, S. Davino ${ }^{1,2}$}

${ }^{1}$ Department of Agricultural, Food and Forest science, University of Palermo, Viale delle Scienze, bld 5, 90128 Palermo, Italy; ${ }^{2}$ Institute for Sustainable Plant Protection, National Research Council (IPSP-CNR), Strada delle Cacce 73, 10135 Turin, ItalyE-mail: salvatore.davino@unipa.it

Tomato brown rugose fruit virus (ToBRFV) represents a serious risk for tomato cultivation due to the ability of the virus to transmit by seeds, plantby-plant contact and manipulation. In order to rapidly detect ToBRFV, a highly sensitive and reliable RT-qPCR assay was developed. Multiple alignment of four full-length genomic sequences of ToBRFV retrieved from GenBank and one more characterized in our lab (isolate ToB-SIC 01/19) was carried out to design a primer pair and a specific TaqMan MGB probe targeting the movement protein gene. In vitro analysis using PrimerBLAST algorithm showed no match with other organisms. To validate the specificity of RT-qPCR assay, 25 samples that included healthy tomato, plants infected with other tobamoviruses, and plants infected with ToBRFV isolate ToB-SIC1/19, were used. Only total RNA-ToB-SIC1/19 gave positive signal, while other tobamoviruses and healthy tomato plants yielded no signal. To determine sensitivity of the RT-qPCR a standard curve was generate using in vitro RNA $(+)$ transcripts. Standard curve showed a strong linear relationship $\left(\mathrm{R}^{2}=0.9997\right)$ and an amplification efficiency of $100 \%$. Subsequently, four different type of samples preparation were tested using the same samples previously described: i) Immunocapture in Real Time PCR Multiwell; ii) Total RNA extraction; iii) Direct crude extract; iv) Tissue-disk crude extract. The four different methods gave positive and interesting results showing that all methods are useful for subsequently RT-qPCR. RT-qPCR for the diagnosis of ToBRFV could represent a useful tool for monitoring strategies of tomato crops.

\section{Effect of polysaccharide Tramesan as biostimulant and resistance inducer on different plants}

A. Parroni ${ }^{1}$, P. Cescutti ${ }^{1}$, C. Fanelli ${ }^{1}$, R. Rizzo ${ }^{1}$, S. Carpenito ${ }^{2}$, N. Scotti $^{3}$, L. Gualtieri ${ }^{4}$, M. Ruocco ${ }^{4}$, M. Reverberi $^{1}$

${ }^{1}$ Dipartimento di Biologia Ambientale, Università Sapienza, P.le Aldo Moro 5, 00165 Roma; ${ }^{2}$ Centro OASIS srl, via Bosco Faino, Torre le Nocelle (AV); ${ }^{3}$ Istituto di Bioscienze e Biorisorse (IBBR-CNR), Via Università, 133 Portici (NA); ${ }^{4}$ Istituto per la Protezione Sostenibile delle Piante (IPSP-CNR), Via Università, 133 Portici (NA). E-mail: massimo.reverberi@uniromal.it

Tramesan is a novel $\alpha$-heteropolysaccharide with a molecular weight of 23000 Da produced by the nontoxic and edible basidiomycete Trametes versicolor, a source of different bioactive compounds utilized in medicine and, recently, in agriculture. Tramesan has been found secreted in the culture filtrate of $T$. versicolor and its chemical structure was characterized by mass spectrometry and NMR analyses. Previous tests demonstrated an inhibiting effect of Tramesan on mycotoxin biosynthesis by different mycotoxigenic fungi (Aspergillus spp and Fusarium spp) in vitro as well as in vivo. Here is reported the effects of Tramesan both as horticultural plants biostimulant (different types of lettuce, tomato, rocket salad) and as an inducer on defence genes such as PAL, PR9, CERK1, RBOH, PR1 in durum wheat. Furthermore, in order to obtain the best results on plants, the best conditions for the Tramesan production and the best Tramesan concentrations to use were assessed. To this aim, also the rough filtrate of T. versicolor was assayed, at different concentrations, first in vitro and subsequently in planta. The results were promising, with a significant increment of biomass produced and a clear biostimulant effect. This work was supported by funds of the project PON POSSIBILE

\section{Monitoring the sensitivity of Fusarium graminearum against four demethylase inhibitors}

\section{Pasquali ${ }^{1}$, M. Pallez-Barthel ${ }^{2}$, M. Beyer $^{2}$}

${ }^{1}$ DEFENS- University of Milan, Via Celoria 2, 20133 Milano, Italy; ${ }^{2}$ Luxembourg Institute of Science and Technology, rue du Brill 41Belvaux, Luxembourg.E-mail: matias.pasquali@unimi.it

Demethylase inhibitors (DMIs) are the most important and effective registered chemicals for controlling Fusarium head blight caused by $F$. graminearum in cereals. The level of sensitivity of a representative set of $F$. graminearum strains $(N=20)$, collected over the period 1981-2012 in Europe and North America against four DMIs (cyproconazole, propiconazole, tebuconazole, tioconazole) was assessed using a microplate test. Median molar EC $_{50}$ values varied $113 \mathrm{X}$ among DMIs with cyproconazole and tebuconazole being the least and the most effective ones, respectively. No complete resistance was detected but $\mathrm{EC}_{50}$ s values varied approximately $10 \mathrm{X}$ from the most to the least sensitive strain. A correlation between the sensitivity levels of the strains to tebuconazole and propiconazole was observed $(\mathrm{r}=0.597 P<0.01)$, while no significant relationships were observed for the other DMIs tested here. The role of previously described single nucleotide polymorphisms (SNPs) located in 10 genes which were reported to influence propiconazole sensitivity was investigated. Our results indicate that sensitivity differences to DMIs in the strains analyzed is determined by mechanisms that are partially specific to each DMI molecule.

Description of a plant-beneficial Pseudomonas syringae strain: colonization, plant-growth promotion and biocontrol effects

A. Passera ${ }^{1}$, S. Compant ${ }^{2}$, P. Casati ${ }^{1}$, M.G. Maturo ${ }^{3}$, G. Battelli ${ }^{4}$, F. Quaglino $^{1}$, L. Antonielli ${ }^{2}$, D. Salerno ${ }^{5}$, M. Brasca ${ }^{4}$, S.L. Toffolatti ${ }^{1}$, F. Mantegazza $^{5}$, M. Delledonne ${ }^{3}$, B. Mitter ${ }^{2}$

${ }^{1}$ Department of Agricultural and Environmental Sciences - Production, Landscape, Agroenergy, Università degli Studi di Milano, Milan, Italy; ${ }^{2}$ Center for Health \& Bioresources, AIT Austrian Institute of Technology GmbH, Tulln, Austria, ${ }^{3}$ Functional Genomics Laboratory, Department of Biotechnology, University of Verona, Verona, Italy; ${ }^{4}$ Institute of Sciences of Food Production, Italian National Research Council, Milan, Italy; ${ }^{5}$ Department Medicina e Chirurgia, Università degli Studi di MilanoBicocca, Vedano al Lambro, Italy.E-mail: alessandro.passera@ unimi.itv

Plants develop in a microbe-rich environment and must interact with a plethora of microorganisms, both pathogenic and beneficial. The genus Pseudomonas has received particular attention because of its strains that can show beneficial or pathogenic effects on plants, such as the model organisms $P$. fluorescens and $P$. syringae. The present study aims to 
compare plant-beneficial and pathogenic strains belonging to the $P$. syringae species to get new insights into the distinction between the two types of plant-microbe interactions. In assays carried out under greenhouse conditions, $P$. syringae pv. syringae strain 260-02 was shown to promote plant-growth and to exert biocontrol of $P$. syringae pv. tomato strain DC3000 in tomato and pepper plants, have a biocontrol effect against Botrytis cinerea in different plant hosts (tomato, lettuce, and grapevine), and have a biocontrol ability against cymbidium ringspot virus in pepper plants. P. syringae strain 260-02 also had a distinct volatile emission profile, as well as a different plant-colonization pattern, visualized by confocal microscopy and $g f p$ labelled strains, compared to strain DC3000. The study characterizes strain 260-02 as an interesting organism to employ in understanding the differences between pathogenic and beneficial strains, and opens new, interesting perspectives for future studies in the field of plant-microbe interactions.

\section{MP102-LABINABAG: development of a prototype molec- ular diagnostic kit for the on-site detection of} Macrophomina phaseolina using a portable PCR platform

\section{S. Pecchia, D. Da Lio, A. Vinci, C. Pugliesi, M. Fambrini, G. Puntoni}

Department of Agriculture, Food and Environment, University of Pisa, Via del Borghetto 80, 54124 Pisa, Italy. E-mail: susanna.pecchia@unipi.it

The early diagnosis of plant pathogens from plant and soil samples needs a simple, rapid and cost-effective test, without the use of sophisticated and expensive equipment and reagents. In this study, the soil-borne pathogen Macrophomina phaseolina is used to demonstrate the potential for on-site molecular detection of the prototype diagnostic kit developed. This was achieved using a rapid and simple protocol comprising of a magnetic bead-based nucleic acid extraction, a portable PCR platform and a final NALFIA assay with a generic lateral flow device (LFD). The portable PCR platform powered by an external battery pack and all materials and reagents for an accurate on-site detection of M. phaseolina within $2 \mathrm{~h}$ were included in a professional bag. The DNA extraction method from infected plant and soil material can be completed within $45 \mathrm{~min}$, and does not require centrifugation steps, organic solvents, or the use of liquid nitrogen for sample homogenization. A procedure for lyophilizing PCR reagents was developed to allow their storage and transportation at room temperature and their use in the field. Formulation development, trial freeze drying cycle and post-process analyses were performed in collaboration with the Biopharma Technology Company. DNA extracts were tested by PCR, which is completed in just $1 \mathrm{~h}$, using patented primers for M. phaseolina labelled with biotin and FITC at the $5^{\prime}$ end. The results obtained were comparable to those of PCR testing in the laboratory. Research to develop this platform for the detection of other important plant pathogens is ongoing in our laboratory.

This work was supported by the University of Pisa, Bando Dimostratori Tecnologici 2018, D.R.1937/18 del 6.11.2018, Progetto MP102LABINABAG

\section{Pseudomonas sp. as a new potential biocontrol agent against Heterobasidion species attacking conifers in Europe}

\section{Pellicciaro, L. Giordano, G. Lione, F. Sillo, P. Gonthier}

University of Torino, Department of Agricultural, Forest, and Food Sciences (DISAFA), Via Largo Paolo Braccini 2, I-10095, Grugliasco, Italy.E-mail: paolo.gonthier@unito.it
The Heterobasidion annosum species complex comprises five fungal species causing the most destructive root and butt rots on conifers in the northern hemisphere. Effective control strategies against these fungi include stumps treatments with either chemical (e.g. urea) or biological products consisting of inocula of fungi competing with Heterobasidion spp. The only biological products registered for use against these pathogens are based on Phlebiopsis gigantea. The present study aims to test the efficacy in vitro of the commercially available bio-fungicide Proradix ${ }^{\circledR}$ (Pseudomonas sp. DSMZ 13134) against the four species of Heterobasidion currently present in Europe, i.e. the three native species and the non-native invasive one $H$. irregulare. Dual culture assays of antagonist and pathogen (five isolates per species) were implemented to assess the mycelial growth inhibition on two culture media at two different temperatures. The effects of volatile organic compounds (VOCs) and cell-free filtrates $(\mathrm{CFF})$ were investigated as well. In dual culture experiments, Proradix ${ }^{\circledR}$ significantly $(\mathrm{P}<0.05)$ reduced the growth of Heterobasidion spp. However, the efficacy was variable depending on Heterobasidion species and tested conditions. While the CFF at $80 \%$ $(\mathrm{v} / \mathrm{v})$ concentration prevented both mycelial growth and spore germination of the tested isolates, VOCs seemed to play a minor role in the inhibition of Heterobasidion spp. Results indicate that Proradix ${ }^{\circledR}$ and its CFF are effective in vitro against Heterobasidion spp. If this efficacy will be confirmed by further experiments in controlled conditions and in the field, Proradix ${ }^{\circledR}$ could become an option for the management of forests challenged by Heterobasidion spp. in Europe.

Internal brown spot, a significant physiological disorder of potato tubers: agronomic advances for the prevention

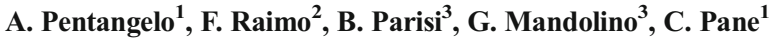 \\ ${ }^{1}$ Consiglio per la Ricerca in Agricoltura e l'Analisi dell'Economia Agraria, Centro di Ricerca Orticoltura e Florovivaismo, via Cavalleggeri, 25 - 84098 Pontecagnano Faiano (Salerno); ${ }^{2}$ Consiglio per la Ricerca in Agricoltura e l'Analisi dell'Economia Agraria, Centro di Ricerca Cerealicoltura e Colture Industriali (Laboratorio di Caserta), via Torrino, 2 - 81100 Caserta, Italy; ${ }^{3}$ Consiglio per la Ricerca in Agricoltura e l'Analisi dell'Economia Agraria, Centro di Ricerca Cerealicoltura e Colture Industriali (sede di Bologna), via di Corticella, 133 -40128 Bologna, Italy.E-mail: catello.pane@crea.gov.it}

Internal brown spot or IBS is a significant physiological nonparasitic disorder of potato tubers. Parenchymal tissues are interested by the appearance of isolated rust colored patches around the vascular ring that can, then, join and necrotize with symptoms' worsening. IBS affected tubers are completely unmarketable defecting in quality, sensory and other technological features. IBS aetiology is considered very complex; although several studies indicated the relevance of genetic, nutritional and environmental factors, it has not yet been completely understood. The cell-wall structural collapse in the tubers, due to the migration of calcium ions sucked by starved hot and/or water stressed leaves via acropetal xylem flux, is, to date, the most accredited hypothesis of IBS triggering. Summarizing results from several field trials carried out in the recent years in Campania region, we could get advances for an effective IBS agronomic management. The experimentations focused on the use of non-sensitive/sensitive cultivars, target fertilization, water supply modulation, mycorrhizal inoculation and harvest timing. Findings indicated that IBS increased with tuber size and symptoms quickly developed at the end of the growing cycle, when tuber bulking stage is in progress and hot and drought burst. On the other hand, agronomic efforts aimed at modulating tuber size and 
canopy vigour, and escaping detrimental stress conditions may play a crucial role in the containment of the disorder. Tools for IBS prevention include the varietal choice addressed towards nonsensitive genotypes, the modulation of the external inputs for a more balanced plant development, the anticipation of sowing and maturation or haulm killing practices.

\section{Sequencing of avirulent strains of $F$ usarium fujikuroi con- tributes to clarify the pathogenicity mechanism on rice}

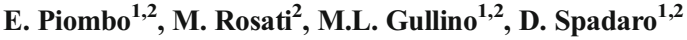 \\ ${ }^{1}$ A GROINNOVA - Centre of Competence for the Innovation in the Agro- \\ environmental Sector, University of Turin, Largo Braccini 2, 10095 \\ Grugliasco, TO, Italy; ${ }^{2}$ Department of Agricultural, Forestry and Food \\ Sciences (DISAFA), University of Turin, Largo Braccini 2, 10095 \\ Grugliasco,TO, Italy.E-mail: davide.spadaro@unito.it
}

Bakanae, caused by Fusarium fujikuroi, is one of the most important diseases of rice. Crop losses are largely depending on climate and rice cultivars, varying from $3 \%$ to $75 \%$. The most common symptoms are abnormal height, thin leaves and empty grains, but some strains have been known to induce stunting and withering instead. The induction of elongation has been linked to the production of gibberellins, but the mechanisms of pathogenicity by F. fujikuroi are unclear. Despite the high number of $F$. fujikuroi strains deposited in online databases, no genome of proven avirulent strains is available, and no comparative genomics study between virulent and avirulent isolates has been ever conducted. Through pathogenicity trials, we confirm the avirulence of F. fujikuroi strain SG4, and the low virulence of strain $\mathrm{C} 2 \mathrm{~S}$. Their genomes were sequenced by Illumina MiSeq, and compared to the genome of the virulent strain I1.3. Two methods are used to compare the genomes: gene prediction and SNP calling. Gene prediction is conducted with MAKER, finding 13,527, 14,795 and 14,534 genes for I1.3, SG4 and C2S, respectively. SNP calling is performed using the genome of F. fujikuroi strain IMI 58289 as reference. Genes present in I1.3 and not in SG4, as well as genes with putatively impactful polymorphisms in SG4 and not in I1.3, are identified and analysed, producing a set of 13 genes putatively related to pathogenicity in F. fujikuroi, many of which are related to plant cell wall degradation.

\section{Search for resistance response to Zymoseptoria tritici in wheat landrace from world different areas}

\author{
S. Pipponzi ${ }^{1}$, J. Novi ${ }^{1}$, T.C. Marcel $^{2}$, S. Gelisse ${ }^{2}$, M. Maccaferri ${ }^{1}$, A. \\ Prodi $^{1}$
}

${ }^{1}$ Department of Agricultural Science DISTAL, Alma Mater Studiorum University of Bologna, Viale G. Fanin, 44-46, 40127, Bologna, Italy; ${ }^{2}$ UMR BIOGER, INRA, AgroParisTech, Université Paris-Saclay, 78850 Thiverval-Grignon,France.E-mail: antonio.prodi@unibo.it

Zymoseptoria tritici is a wheat foliar pathogen causing Septoria tritici blotch (STB), the most damaging disease of wheat in Europe, causing yield losses of up to $50 \%$. During the cropping season, Z. tritici disseminates over short distances by producing pycnidiospores generated by asexual reproduction. A collection of 175 landraces of Triticum durum was gathered from 32 states worldwide. This set was infected with four Mediterranean strains of the fungus isolated from T. durum (two of them were isolated in Italy, one in France and one in Tunisia), with the aim of identify some resistant genotypes. The inoculum was spread on the leaf surface and plants were kept at a temperature of $18-20^{\circ} \mathrm{C}$ and $80 \%$ of humidity. Symptoms evaluation was performed after 20 and 26 days based on the necrotic and sporulating leaf area extension. Consequently, AUDPC values were obtained. A 90K SNP array was performed on the genotypes. Based on the similarity, the accessions were divided into six groups. To each group correspond one well defined geographical area. Taking into account the extension of the sporulating leaf area, it was established that about $10 \%$ of the seedling genotypes were resistant to three or four different $Z$. tritici strains. AUDPC values and the groups obtained with a 90KSNP array, the accessions from Greece and Crete Instead, considering the appear to be the most resistant to all the four Z. tritici stains used in the trials.

\section{Production of beneficial microbes with improved proper- ties}

A. Pironti ${ }^{1}$, R. Marra ${ }^{1}$, A. Staropoli ${ }^{1,2}$, G. Manganiello ${ }^{1}$, N. Lombardi $^{1}$, F. Vinale ${ }^{1,2}$, S.L. Woo ${ }^{2,3}$, M. Lorito ${ }^{1,2}$

${ }^{1}$ University of Naples Federico II, Department of Agricultural Sciences, Via Università 100, 80055 Portici (NA), Italy; ${ }^{2}$ National Research Council, Institute for Sustainable Plant Protection, Via Università 133, 80055 Portici (NA), Italy; ${ }^{3}$ University of Naples Federico II, Department of Pharmacy, Via D. Montesano 49, 80131 Naples, Italy. E-mail: angela.pironti@unina.it

The efficient use of biocontrol agents (BCA) and the development of protocols to produce active propagules are fundamental aspects in the establishment of a biological control program. New BCAs may be obtained from suppressive soils or using genetic manipulation techniques, such as transformation, mutagenesis or protoplast fusion $(\mathrm{PF})$. Numerous studies used PF to make intra- and inter-species crosses of Trichoderma fungi. In this work we used PF to produce Trichoderma hybrids with improved efficacy compared to parental strains in terms of biocontrol ability, production of bioactive metabolites and/or promotion of plant growth. The progeny obtained by recombinant fusion between two Trichoderma strains (T. harzianum strain M10, T. virens strain GV41) was screened by testing biocontrol activity against pathogenic fungi (Rhizoctonia solani, Botrytis cinerea) and plant growth promotion ability. About $80 \%$ of tomato plants treated with the hybrid mixture was not affected by $R$. solani and increased root length compared to control and parentals. Fifty monosporic cultures were isolated from the rhizosphere of tomato plants treated with Trichoderma hybrid mixture and new strains named A5 and A3 were selected. Metabolomic analysis of hybrids detected compounds that were not found in parental strains liquid cultures. Further characterization of fungal hybrids is in progress.

Isolation, molecular characterization and aflatoxigenic potential of Aspergillus flavus strains isolated from corn conserved as silage

\footnotetext{
S. Prencipe ${ }^{1,2}$, G.R. Meloni ${ }^{1,2}$, F. Ferrero ${ }^{1}$, G. Borreani ${ }^{1}$, M.L. Gullino $^{1,2}$, D. Spadaro ${ }^{1,2}$

${ }^{1}$ DISAFA - Dipartimento di Scienze Agrarie, Forestali ed Alimentari, Università degli Studi di Torino, Grugliasco (TO), Italy; ${ }^{2}$ Centro di competenza per l'Innovazione in campo agroambientale (AGROINNOVA), Università degli Studi di Torino, Grugliasco (TO), Italy.E-mail: davide.spadaro@unito.it
}

The presence of A. flavus and aflatoxins (AFs) in corn silage could constitute a problem for human and animal safety. During 2016-2018 an extensive sampling of Aspergillus Section Flavi from the field, at silos 
opening and during aerobic deterioration was conducted. Sampling was carried out from high moisture corn, wet grain and whole crop corn. Isolates were identified through morphological and molecular analyses: 117 were identified as A. flavus and 39 as A. orzae var. effusus. The highest incidence of $A$. flavus was found in aerobic deteriorated silage, and, at lower concentration, in the field and in conserved silages. Fortyfive over 117 strains of A. flavus were further characterized through phylogenetic and chemical analyses. The phylogenetic analyses showed an intraspecific variability and the strains clustered in three groups. The aflatoxigenic potential was tested in vitro by incubating the strains at 25 ${ }^{\circ} \mathrm{C}$ and $35^{\circ} \mathrm{C} .75 .56 \%$ of strains were able to produce AFs at $25^{\circ} \mathrm{C}$ while only $49 \%$ at $35{ }^{\circ} \mathrm{C}$. A higher production of AFs was observed at $25^{\circ} \mathrm{C}$ with a mean production of $5.33 \mu \mathrm{g} / \mathrm{g}$ for AFB1 and $0.48 \mu \mathrm{g} / \mathrm{g}$ AFB2. Differently at $35^{\circ} \mathrm{C}$, the production was $0.044 \mu \mathrm{g} / \mathrm{g}$ and $0.0014 \mu \mathrm{g} / \mathrm{g}$ for $\mathrm{AFB} 1$ and $\mathrm{AFB} 2$ respectively. This study shows the presence of $A$. flavus from field to silage and air exposure of corn silage, the high aflatoxigenic potential of the strains, and underline the need to set up new techniques to control the development of aflatoxigenic fungi in order to reduce the potential risk of mycotoxins contamination.

This work was supported by Fondazione Cassa di Risparmio di Cuneo (progetto SOST-MILK: Emerging pests of corn and sustainability of Piedmontese milk production chain).

\section{EF1 $\alpha$-based detection and quantification of Venturia inaequalis in leaves, fruits and air samples by using real- time PCR (TaqMan $\left.{ }^{\circledR}\right)$ assay}

\section{S. Prencipe ${ }^{1,2}$, F. Sillo ${ }^{1}$, M.L. Gullino ${ }^{1,2}$, D. Spadaro ${ }^{1,2}$}

${ }^{I}$ DISAFA - Dipartimento di Scienze Agrarie, Forestali ed Alimentari, Università degli Studi di Torino, Grugliasco (TO), Italy; ${ }^{2}$ Centro di Competenza per l'Innovazione in campo agroambientale (AGROINNOVA), Università degli Studi di Torino, Grugliasco (TO), Italy.E-mail: davide.spadaro@unito.it

Venturia inaequalis is responsible of apple scab on Malus $\mathrm{x}$ domestica. In this study, we developed and validated a high sensitive real-time PCR (TaqMan $\left.{ }^{\circledR}\right)$ assay for the detection and quantification of $V$. inaequalis in apple leaves, fruits and in air samples. The assay was developed using specific primers and probe targeting the translation elongation factor 1- $\alpha$ gene. The primer pairs were first investigated in conventional end-point PCR and then using SYBR Green I technology. The developed assay was compared to a published method based on TaqMan technology and validated for specificity, selectivity, repeatability and reproducibility. Twenty fungal species, representing the most common species present in the field, including $V$. asperata, recently reported on apple in Italy, were tested. An amplicon (86 bp) was amplified using the selected primers and probe. The detection limit was more sensitive than those obtained with previously published methods. No influence on $V$. inaequalis amplification was found when the assay was tested for selectivity using DNA from cultivars 'Ambrosia', 'Golden Delicious' and 'Gala'. The assay was able to detect and quantify the pathogen in symptomatic and asymptomatic leaves and fruits, both in susceptible and resistant cultivars. Additional analyses were performed on air samples, and the estimated counts obtained by TaqMan assay showed a good correlation $\left(\mathrm{R}^{2} 0.8186\right)$ with $V$. inaequalis counts using optical microscope. The developed assay is a useful tool for early detection of $V$. inaequalis in asymptomatic samples, able to improve apple scab management.

This work was supported by Fondazione Cassa di Risparmio di Cuneo (progetto SMARTAPPLE - Innovative and SMART technologies for sustainable apple production).
In vivo antibacterial activity of two essential oil based products to control fire blight

\author{
M.R. Proto, D. Baldo, G. Filippini, C. Ratti, P. Minardi, P. Mattarelli, \\ E. Biondi
}

Department of Agricultural and Food Sciences (DISTAL), Alma Mater Studiorum - University of Bologna, V. le Fanin, 44, Bologna, 40127, Italy. E-mail: enrico.biondi3@unibo.it

The products VitiBioSap 458 Plus ${ }^{\circledR}$ (VBS458) and VitiBioSap ES Plus ${ }^{\circledR}$ (VBSES) based on essential oil mixture, $\mathrm{Cu}^{++}(3.5 \%), \mathrm{Zn}^{++}$ $(1 \%)$ and the MicroSap ${ }^{\circledR}$ component (Patent $N^{\circ}$ EP307103928.09.2016), were assayed in vivo against Erwinia amylovora (Ea), the etiological agent of the bacterial fire blight. Detached flowers and immature fruitlets of pear were pre-treated with VBS458 (1-2\%) or VBSES (2-3\%), and inoculated with an E $a$ aqueous suspension ( $c a$. $10^{6} \mathrm{CFU} / \mathrm{ml}$ ); the disease incidence was evaluated within 6 days after the inoculation. Moreover, the population dynamics of an $E a$ rifampicin resistant mutant strain ( $E a$ 273R1) was evaluated up to 4 days on detached apple flowers, pre-treated with the above products. In addition, at each detection time, the flowers were fixed in 5\% glutaraldehyde solution, dehydrated in ethanol and processed by Critical Point Dryer. The samples, after gold sputter coating, were observed by Philips SEM 515 scanning electron microscope at $20 \mathrm{kV}$. Sterile distilled water (SDW) and streptomycin sulphate (STR) were used as negative and positive controls, respectively.

In VBSES- and VBS458-treated pear flowers, the disease incidence was significantly reduced (12-30\% and $12-50 \%$, respectively) compared to that detected in SDW-treated flowers (88-100\%). In pear fruitlets treated with the products, the disease incidence resulted between $19 \%$ and $46 \%$, similar to that on STR-treated pears (30\%). Furthermore, the Ea population was significantly lowered in VBSES- and VBS458-treated apple flowers, in comparison to that of SDW-treated control samples.

\section{Study of biovar-specific determinants and role of commu- nication with host plant in Pseudomonas syringae pv. actinidiae virulence}

M.R. Puttilli ${ }^{1}$, A. Regaiolo ${ }^{1}$, T. Colombo ${ }^{1}$, V. Maurizio $^{1}$, T. Libardi ${ }^{1}$, V. Venturi ${ }^{2}$, G. De Vescovi ${ }^{2}$, A. Polverari ${ }^{1}$, E. Vandelle $^{1}$

${ }^{1}$ Laboratory of Phytopathology, Department of Biotechnology, University of Verona, Verona, Italy; ${ }^{2}$ International Centre for Genetic Engineering and Biotechnology (ICGEB), Trieste, Italy. E-mail: elodiegenevieve.vandelle@univr.it

Kiwifruit bacterial canker caused by Pseudomonas syringae pv. actinidiae $(P s a)$ is a quarantine plant disease that is threatening global kiwifruit industry, where Italy plays a leading role worldwide. Currently, control measures are mainly based on the use of copper, for which the European Union has concerns due to its possible negative environmental effects. Moreover, Psa resistance to copper is already emerging in several countries. Such a scenario would rapidly leave kiwifruit growers with no chance to efficiently control the pathogen.

We are currently attempting to decipher molecular signals involved in $P s a$ virulence induction and promotion. This will allow the identification of targets for designing new control strategies based on the inhibition of the infection process, instead of killing bacteria, thus averting the appearance of new antibiotic resistances, in accordance with the new trend of bacterial disease control also developed in medical field. 
To that purpose, we produced a $P s a$-specific microarray chip carrying whole genomes of Psa strains of the best-studied biovars (1, 2 and 3). Microarray analyses were performed with wild-type strains for a deep investigation of biovar-specific responses to provide insights into the molecular mechanisms possibly controlling Psa aggressiveness. Moreover, to decrypt the molecular signals governing bacteria-plant communication and to identify molecules inhibiting $P s a$ virulence, a chemical genomics approach was applied using a reporter gene system for monitoring the expression of the virulence targets during Psa growth in presence of kiwifruit extracts.

\section{Deletion of genes encoding relevant Pyricularia oryzae polysaccharide degrading enzymes by CRISPR-Cas9 ge- nome editing and characterization of mutants to identify new virulence factors}

A. Quarantin ${ }^{1}$, S. Tundo ${ }^{1}$, R. Govind ${ }^{1}$, T.-T. Nguyen $^{2}$, C. Castiglioni ${ }^{1}$, F. Favaron ${ }^{1}$, M.H. Nguyen ${ }^{2}$, L. Sella ${ }^{1}$

${ }^{1}$ University of Padova, Department of Land, Environment, Agriculture and Forestry, Viale dell'Università 16, 35020, Legnaro (PD), Italy; ${ }^{2}$ Duy Tan University, K7/25 Quang Trung, Da Nang, Vietnam. E-mail: alessandra.quarantin@unipd.it

By the end of the next decade, rice production will have to increase in order to support the ever-increasing world population. This goal is difficult to achieve due to the rice blast disease, caused by the pathogenic fungus Pyricularia oryzae, which contributes to reduce annually by $10-30 \%$ total rice production. The main objective of the project, which is part of the Scientific and Technological Cooperation Agreement between Italy and Vietnam, is the development of new methods to control rice blast disease in order to increase food production. In the early stages of the infection process, $P$. oryzae forms the infection structure appressorium to break plant cuticle and expresses many polysaccharide degrading enzymes. Enzymes highly expressed during appressorium formation, such as chitin synthase, chitinase, ligninase and 1,3-beta-glucanases, are under investigation as virulence factors and possible targets for plant inhibitors. In particular, a P. oryzae chitinase gene has been knocked-out by target homologous recombination using the $P$. oryzae non-homologous DNA-end joining deficient mutant strain. Pathogenicity assays on barley and rice seedlings with the disrupted mutants are in progress. To accelerate targeted mutation of the genes of interest, we are also applying the innovative ribonucleoprotein-CRISPRCas9 genome editing system. In particular, we are exploiting a modified method by co-transforming fungal protoplasts with a purified Cas 9 endonuclease, a synthesised single guide RNA and a donor DNA containing homologous flanking regions and a selectable marker. Donor DNAs are produced following a dual approach, by classic cloning and by fusion PCR.

\section{Sensitivity to chitosan and chitosan nanoparticles by three Colletotrichum species belonging to $C$. acutatum species complex}

P. Quattrocelli ${ }^{1}$, G. Puntoni ${ }^{1}$, S. Bianchi ${ }^{2}$, V. Castelvetro ${ }^{2}$, R. Baroncelli ${ }^{3}$, S. Pecchia ${ }^{1}$

${ }^{1}$ Department of Agriculture, Food and Environment, University of Pisa, Via del Borghetto 80, 54124 Pisa, Italy; ${ }^{2}$ Department of Chemistry and Industrial Chemistry, University of Pisa, Via G. Moruzzi 13, 56124 Pisa, Italy; ${ }^{3}$ Department of Microbiology and Genetics, University of Salamanca, Campus de Villamayor, Calle Del Duero, Villamayor, España.E-mail: susanna.pecchia@unipi.it
Chitosan $(\mathrm{CH})$ is one of the most studied biomaterials in nanotechnology due to its unconstrained biological properties like antimicrobial and plant growth regulatory activity, biodegradability, biocompatibility and non-toxicity to humans. Although many reports of the application of chitosan in agriculture are available, much work has to be done using chitosan nanoparticles (CHNPs) to fully understand their true potential. The advantages of CHNPs over bulk materials relies in their small size and large surface area. CHNPs exhibited higher antimicrobial activity than bulk materials and their cellular uptake into cells was higher than that of chitosan molecules. In this study, antifungal effects of chitosan nanoparticles (CHNPs) and commercial chitosan (ChitoPlant $\left.{ }^{\circledR}\right)$ against Colletotrichum lupini, C. nymphaeae and C. acutatum sensu stricto were compared using a microdilution assay in 96-well microtiter plates. Fungi were isolated from lupin, laurel and fig respectively and were molecularly identified using ITS and GAPDH sequences. CHNPs were synthesized via the ionotropic gelation method using sodium tripolyphosphate (TPP) as cross-linking agent and were characterized by size (DLS), and zeta potential analysis. The size of CHNPs was approximately $98 \mathrm{~nm}$ with a zeta potential of $12 \mathrm{mV}$. CHNPs (5000 ppm) were found most effective showing 90-100\% growth inhibition of the three Colletotrichum spp. which showed a tolerance to $\mathrm{CH}$ (5000 ppm) with percentage of growth inhibition ranging from 5 to $29 \%$. The $260 \mathrm{~nm}$ absorbing cellular material increased significantly when compared to untreated control samples indicating a membrane damage mediated by CHNPs. The results suggest that CHNPs have remarkable potential for further in vivo applications.

\section{Monitoring of the presence of Phyllosticta citricarpa in Calabria}

\section{Riccioni, L. Orzali, M.T. Valente, E. Marinelli}

CREA-DC, Research Centre for Plant Protection and Certification. Email: luca.riccioni@crea.gov.it

Phyllosticta citricarpa (McAlpine) Aa, is a quarantine fungus causing "citrus black spot" disease. It is a leafspotting and fruitblemishing fungus affecting Citrus, Poncirus and Fortunella and their hybrids. Citrus limon is particularly susceptible and thus it is usually the first Citrus species to show symptoms of the disease once the pathogen is introduced into a new area. Citrus black spot is now present in some citrus-producing areas of Africa, Asia, Australia, and North and South America. The organism has not been reported from Europe until a recent report in 2017. After the report of the presence of P. citricarpa in Calabria, as well as in one site in Greece and one in Portugal in 2017, a network of monitoring in Italy and Greece has been built, funded by European Food Safety Authority (EFSA), in order to confirm the presence of the quarantine pathogen fungus or to declare those areas as free area. The monitoring is based on a weekly collection of the fungus spores using a volumetric spore trap (Burkard Manufacturing Co Ltd) set up near the same site of the first report, and of a monthly 
collection of fruits and leaf litter from the lemon trees present in the near area. Both samples, the weekly tape from the spore trap and the vegetable samples are analysed by real-time PCR with specific primers.

The present project is funded by EFSA

\section{Distribution of Phytophthora oleae in southern Italy}

\section{Riolo ${ }^{1}$, M. Evoli ${ }^{1}$, L. Schena ${ }^{2}$, F. Aloi ${ }^{1,3}$, E. Santilli ${ }^{4}$, D. Ruano-} Rosa $^{5}$, G.E. Agosteo ${ }^{2}$, A. Pane ${ }^{1}$, F. La Spada ${ }^{1}$, S.O. Cacciola ${ }^{1}$

${ }^{1}$ Dipartimento di Agricoltura, Alimentazione e Ambiente, University of Catania, via S. Sofia 100, 95123 Catania, Italy; ${ }^{2}$ Dipartimento di Agraria, University Mediterranea of Reggio Calabria, Feo di Vito s.n.c., 89122 Reggio Calabria, Italy; ${ }^{3}$ Department of Agricultural, Food and Forest Sciences, University of Palermo, Viale delle Scienze, Building 4, 90128 Palermo, Italy: ${ }^{4}$ Council for Agricultural Research and Economics - Research Centre for Olive, Citrus and Tree Fruit (CREA-OFA), Contrada Li Rocchi-Vermicelli, 83 - 87036 Rende (CS), Italy; ${ }^{5}$ Instituto Tecnológico Agrario de Castilla y León - Unidad de Cultivos Leñosos y Hortícolas, Valladolid,Spain.E-mail:mario-riolo@hotmail.it

Phytophthora oleae, a recently described new homothallic species in clade 2 with an optimum temperature of about $20^{\circ} \mathrm{C}$ and persistent, semi-papillate sporangia, causes occasional outbreaks of rot on mature olive drupes. Its geographical distribution has been investigated in olive orchards in Calabria using carob (Ceratonia siliqua) leaf baiting and selective isolation medium for Phytophthora. Isolates were identified by sequencing the ITS1-5.8S-ITS2 region of rDNA and coxl gene. The species was recovered from rhizosphere soil and fine roots of olive trees in a relatively large area along the Tyrrhenian coast of Calabria with no apparent association with root rot and decline symptoms on the tree canopy. Reports of $P$. oleae in undisturbed naturalized riparian ecosystems in Sicily (where olive is an essential and characteristic component of phytocenosysis) as well as in protected natural area in Spain (where $P$. oleae was associated with root rot of wild olive) raise the questions whether its natural host range is restricted to olive or it is a polyphagous species like its close relatives in clade 2 , and whether it is a recently introduced, invasive pathogen or a naturalized, occasional, weak pathogen in olive stands.

\section{Tolerant and resistant citrus genotypes to Plenodomus tracheiphilus}

\author{
R. Russo ${ }^{1,2}$, E. Nicolosi ${ }^{2}$, S.G. Scaccianoce ${ }^{1}$, A. M. Fichera ${ }^{1}$, M. \\ Caruso $^{1}$, S. Di Silvestro ${ }^{1}$ \\ ${ }^{1}$ CREA - Research Centre for Olive, Tree fruits and Citrus, Corso Savoia \\ 190, 95024, Acireale, Catania, Italy; ${ }^{2}$ Department of Agriculture, Food \\ and Environment, University of Catania, Via Valdisavoia 5, 95123 \\ Catania, Italy. E-mail: riccardo.russo.1991@gmail.com
}

Plenodomus tracheiphilus is the agent of "Mal secco", one of the most injurious disease of citrus that is widespread in different Mediterranean countries. It is a tracheomycotic disease, besides lemon, "Mal secco" pathogen infects, with high yield losses, other citrus species. Identification of citrus genotypes tolerant and resistant to Mal secco is the aim of a phenotypic survey that started in the CREA orchard located in Acireale, Italy where there is lemon and lemon-like germplasm. It was planted in 2002 and today contains 25 lemon clonal selections and autotetraploids, as well as 17 citron and lemon hybrids. Other citrus species reported as tolerant or resistant were also embedded in the survey as reference. All genotypes were replicated twice or three times and were planted in the same field block where the pressure of $P$. tracheiphilus is very high. Phenotyping for "Mal secco" symptoms started in 2018 and was performed twice on the survived clones. A screening by PCR was applied to detect possible sources of $P$. tracheiphilus in old flushes, new flushes and branches. The results showed important sources of tolerance in the germplasm. The pathogen was detected in all lemon clones, many lemon hybrids and citron hybrids, also in trees without clear symptoms, informing us about the ability of some genotypes to recover from the disease. Interestingly, an autotetraploid lemon seems to be immune to the disease under natural pressure, since $P$. tracheiphilus was not diagnosed by PCR and visual screening. Pathogen quantification was performed by Real Time PCR. This work was supported by: Associazione "Limone dell'Etna"

\section{Effects of different viroids mixtures on Navelina ISA 315 grafted on five different rootstocks}

\section{T. Russo, M. Guardo, S. Di Silvestro, G. Varrica, G. Sorrentino}

CREA - Research Centre for Olive, Tree fruits and Citrus, Corso Savoia 190, 95024, Acireale, Catania, Italy.E-mail: tiziana30russo86@gmail.com

Citrus viroids are very harmful pathogens in citrus orchards. With the aim to evaluate their effects and symptoms in a long period, a monitoring was conducted in an experimental citrus field planted and inoculated 25 years ago in Sicily. Observations were carried out on plants of 'Navelina ISA $315^{\prime}$ grafted on five different rootstocks, Robidoux trifoliate orange, Swingle citrumelo CPB 4473, Carrizo CES 2863 citrange, BA 300 citrange and Thomasville citrangequat; the seedlings were inoculated with 3 different citrus viroids complex: [Citrus Exocortis Viroid (CEVd) from Israel]; [CEVd, Hop stunt Viroid (HSVd) and CVd-III]; [CEVd, HSVd, CVd-III and citrus bent leaf viroid (CBLVd)]. The trees were planted in 1994 and since 2002 yield, fruit quality and size were evaluated every year for 10 years. The symptoms (gumming, bark scaling and yellowing) were assessed during until 2017. The results showed not only a strong reduction in the development of BA 300 and Robidoux Poncirus trifoliata, but also greater sensitivity to inocula with a higher percentage of dead (74\%) or symptomatic trees. Other graft combinations showed less sensitivity to viroids, with a higher percentage of productivity and lower percentage of dead (36\%) and symptoms, in particular on Thomasville citrangequat. The Navelina grafted on Citrange carrizo showed a size reduction too. Despite the sensitivity of these rootstocks, especially to CEVd, after 23 years from the start of trial, $46 \%$ of the plants survived. The viroids' damage is not death of the plants but the reduction of development and productivity.

\section{New reports of diseases on soft and durum wheat in Tuscany during the 2018/2019 crop season}

\author{
A. Salerno ${ }^{1}$, G. Carella ${ }^{1}$, M. Nocentini ${ }^{1}$, M. Ricciolini ${ }^{2}$, L. Mugnai ${ }^{1}$ \\ ${ }^{1}$ Department of Agricultural, Food, Environmental and Forestry Science \\ and Technology (DAGRI), Plant pathology and Entomology section, \\ University of Florence, P.le delle Cascine, 28, 50144 Firenze, Italy; \\ ${ }^{2}$ Servizio Fitosanitario Regionale, Regione Toscana, Firenze, Italy. E- \\ mail: laura.mugnai@unifi.it
}

Wheat diseases monitoring has been carried out in Tuscany (Grosseto, Arezzo and Florence province) since 2013 within a collaboration agreement with the Regional Phytosanitary Service for monitoring and alert. The varieties of wheat chosen were both ancient and modern varieties of durum and bread wheat, with organic and/or integrated management. The 2018/2019 
weather season was very atypical. A very dry early spring, with daily temperatures almost always below average, slowed down the phenological development of wheat as well as the most precocious diseases such as septoria (Septoria tritici) and yellow rust (Puccinia striiformis). The below average season was accompanied by very high and continuous precipitation, much above the average. In these exceptional conditions diseases that were not usually reported in the previous years caused severe damage. Head blight (Fusarium spp.), especially on durum wheat, caused severe infections in some areas, due to the coincidence of flowering time with prolonged rainy events. Stem rust (Puccinia graminis f. sp. tritici), which was only very occasionally reported in Tuscany in the last 15 years at least, appeared in early June and caused severe damage to both culms and ears. This opened questions about the race that caused such a sudden and aggressive epidemic in Tuscany, especially following the report of the recent epidemic caused by race TTTTF in Sicily and the increasing spread of Ug99. Furthermore, the presence of two almost forgotten diseases, loose smut (Ustilago tritici) and bunt of wheat (Tilletia caries and possibly other species) was reported occasionally but sometimes, especially in organic cereal cultivation, with high severity.

\section{Detecting downy mildew symptoms on wild rocket leaves by hyperspectral imaging}

\section{Santonicola, D. Villecco, A. Pentangelo, C. Pane}

Consiglio per la Ricerca in Agricoltura e l'Analisi dell'Economia Agraria, Centro di Ricerca Orticoltura e Florovivaismo, via Cavalleggeri, 25 - 84098 Pontecagnano Faiano (Salerno).E-mail: catello.pane@crea.gov.it

Italy is among the major European producers of wild rocket (Diplotaxis tenuifolia) for the high-convenience food chain. Downy mildew caused by the oomycete Peronospora parasitica is a key threat to the greenhouse cultivations, where favourable weather conditions to pathogen occur, causing severe yield losses. Symptoms appear as isolated or confluent irregular brown spots on discoloured rocket leaves. In this case, disease management is very challenging; generally it relies on the chemical control. However, for organic or zero-residue produce it is necessary to develop sustainable alternatives, including the use of digital systems to support farmer's decisions. Hyperspectral imaging is an available technology that allows to increase detection, monitoring and mapping capabilities on cropping systems with the aim of leading site-specific and timely intervention. For this purpose, healthy wild rocket leaves and leaves affected by a different degree of Peronospora infection have been collected from a greenhouse cultivation, and scanned by the hyperspectral sensor, Specim IQ spectral camera covering VIS and NIR regions between 400-1000 nm. A pixel-wise mapping of reflectance in these spectral regions enabled the detection and detailed description of diseased leaf areas. Hyperspectral imaging offers high potential as a non-invasive diagnostic tool for disease detection. Findings are encouraging about futuristic application of optical devices to diagnose downy mildew directly on rocket crops. Work is ongoing, attempting to compute multispectral image analysis algorithm for disease detection and classification.

This work was supported by MIPAAFT, D.M. 36503.7305.2018, AGROFILIERE sub-project (AgriDigit program)

\section{Diffusion and variability of Ciborinia camelliae in Italy}

\author{
M. Saracchi, E.M. Colombo, P. Cortesi, C. Pizzatti, A. Kunova, M. \\ Pasquali
}

DeFENS - Department of Food, Environmental and Nutritional Sciences, University of Milan, Via Celoria 2, 20133 Milan Italy. E-mail: marco.saracchi@unimi.it
Camellia flower blight is a severe disease caused by Ciborinia camelliae Kohn, fungus belonging to Ascomycota family of Sclerotiniaceae. It was first described in Japan in 1919, and afterwards in North America, Europe, Asia and Oceania. In Italy, it was reported for the first time in 2001. Studies carried out on the pathogen are occasional. The aim of this research was to study the diffusion and the diversity of the pathogen population in Italy. The presence of Camellia flower blight was confirmed in Piedmont, Tuscany, and Lazio and was reported for the first time in Emilia Romagna, Lombardy, Liguria, and Campania. The morphophenotype of more than seventy strains from different Italian regions was characterized on four culture media at six temperatures. Based on these data, morphotypes were described showing a significant phenotypic variability within the Italian population. Multigene sequencing and universal primers were used to assess genetic variability among Italian strains. Nucleotide sequences of some informative genomic regions (e.g. ITS, beta-tubulin, and gpdh) showed high uniformity among Italian and European isolates, but significant differences with respect to isolates from China and New Zealand. Our study verified the diffusion of the pathogen in the Italian peninsula and will contribute to better define the species of $C$. camelliae from the genetic point of view. Further studies are warranted to assess the level of variability at the global scale.

\section{The devil wears Trichoderma gamsii: competitive interac- tions with Fusarium graminearum to control Fusarium Head Blight}

\section{S. Sarrocco, G. Vannacci}

Dipartimento di Scienze Agrarie, Alimentari e Agro-ambientali, Università di Pisa. Via del Borghetto 80, 56124 Pisa, Italy. E-mail: sabrina.sarrocco@unipi.it

Fusarium Head Blight (FHB) is a worldwide disease responsible of great yield losses as well as mycotoxin contamination of wheat. Among the almost twenty species listed as causal agents, Fusarium graminearum is one of the most important mycotoxigenic fungi. Thanks to a very efficient saprotrophic phase, this pathogen can survive on cultural debris as perithecia, where ascospores infecting flowers at anthesis are produced. Different managing tools such as resistant cultivars, cropping rotations, tillage and fungicides are used to manage the disease, unfortunately with partially unsatisfying results. The use of beneficial fungi represents an innovative approach to control FHB causal agents and to reduce the risk that mycotoxins enter into the food chain.

Trichoderma gamsii T6085 is well known for its ability to reduce $F$. graminearum growth and mycotoxins production through different mechanisms. In the present work, the most recent results concerning competition for wheat straw by T6085 vs $F$. graminearum are reported, both in terms of biomass and perithecia reduction. Information obtained by a transcriptomic analysis also showed iron as an important competitive factor between $\mathrm{T} 6085$ and this pathogen. Competition not only occurs at a lab-scale but seems to be the mechanism used by T6085 when applied on cultural debris and spikes at anthesis also under field conditions where FHB resulted to be reduced. Herewith obtained knowledge contributes to the development of T6085 as bioactive ingredient of a new biopesticide against FHB.

\section{A global assessment of the burden of pathogens and pests on major food crops}

\section{S. Savary ${ }^{1}$, L. Willocquet ${ }^{1}$, S.J. Pethybridge ${ }^{2}$, P.D. Esker ${ }^{3}$, N. McRoberts ${ }^{4}$, A. Nelson ${ }^{5}$}


${ }^{1}$ AGIR, INRA, Université de Toulouse, INPT, INP-EI Purpan, CastanetTolosan, France; ${ }^{2}$ School of Integrative Plant Science, Cornell University, The New York State Agricultural Experiment Station, Geneva, NY, USA; ${ }^{3}$ Department of Plant Pathology and Environmental Microbiology, Penn State University,University Park, PA, USA; ${ }^{4}$ Plant Pathology Department, University of California, Davis, Davis, CA, USA; ${ }^{5}$ Faculty of GeoInformation Science and Earth Observation, University of Twente, Enschede, the Netherlands.E-mail: Serge.Savary@inra.fr

Plant pathogens and pests cause important crop losses which reduce agricultural production; but they also impact societies and the environment, since they affect economic fabrics and drain environmental resources. What is the magnitude of these effects? The landscape of plant pathology is made up of "stars": potato late blight, rice blast, wheat stripe rust. And therefore, it consists also of neglected diseases - one might think of rice brown spot, of wheat spot blotch, of the virus diseases of cassava, and many others. Measuring quantitatively these impacts would allow gauging the value of better plant health, would enable the clear definition of intervention points, and would generate the necessary baseline for research policies - which are the most important diseases, and why? Despite these needs, there is no global data base on disease crop losses, for a number of reasons. One is the sheer diversity of plant pathogens; another is the difficulty for measurement, combined with metric issues; a third is the cost of field experiments and surveys; and a fourth is the inherent variability of crop health over time, seasons, and space, within field and from plots to continents. This presentation will report a recent effort (to quantify losses in wheat, rice, potato, soybean, and maize globally, through an international expert survey. This work enables better positioning plant pathology with major challenges of our times: global and climate change, food (in)security, and the state of the biosphere.

The authors are grateful to the International Society for Plant Pathology (ISPP) for help in reaching out to experts. The authors thank all experts who have contributed to the online survey. N.M. was partly supported by USDA-NIFA project CA-D-PPA-2131-H.

\section{Etiological studies over kiwifruit decline reveal the in- volvement of both flooding and biotic factors}

\section{F. Savian, R. Musetti, N. Sandrin, P. Ermacora, M. Martini}

University of Udine, Department of Agricultural, Food, Environmental and Animal Sciences, Via Delle Scienze 206-33100 Udine, Italy. E-mail: francesco.savian@uniud.it

In Italy kiwifruit cultivation is facing a new threat due to the recent spreading of a disease named kiwifruit decline. Diseased plants with a collapsed root system go through a sudden and irreversible dieback process. The aetiology of the disease is still unknown, but it seems that waterlogged conditions do play a significant role in the induction of the symptoms and the involvement of some biotic factors is more than probable. To shed light on the aetiology, an experimental trial was set up in a greenhouse with 8-month-old kiwifruit plants combining sterilised and unsterilized soil (from diseased orchards) with two different water managements, optimal and temporary submersion conditions. Symptom monitoring, biometric measurements of leaf and root weight and electron microscopy observations were performed on kiwifruit plants. Moreover, fungal isolation from the roots of diseased plants was achieved on PDA and isolates were identified via molecular tools using the ITS region. Only plants grown in unsterilized soil and submersion conditions showed wilting symptoms. Genera Fusarium and Phytopithium were the most frequently isolated but pathogenicity tests are still ongoing. Nevertheless, our results clearly show that a biotic factor is truly involved in the disease complex together with waterlogging conditions.
Twiching motility and biofilm formation influenced by lipid entities selected by lipid profile of olive orchard

\author{
V. Scala ${ }^{1}$, N. Pucci ${ }^{1}$, M. Salustri ${ }^{2}$, V. Modesti $^{1}$, A. L'Aurora ${ }^{1}$, M. \\ Scortichini $^{3}$, M. Reverberi ${ }^{2}$, S. Loreti ${ }^{1}$ \\ ${ }^{1}$ Council for Agricultural research and the Analysis of Agricultural \\ Economics (CREA), Research Centre for Plant Protection and \\ Certification, Via C.G. Bertero 22, 00156 - Roma, Italy; ${ }^{2}$ Sapienza \\ University, Dept. of Environmental Biology, P.le Aldo Moro 5, 00185 \\ Roma, Italy; ${ }^{3}$ Council for Agricultural research and the Analysis of \\ Agricultural Economics (CREA), Research Centre for Olive, Fruit Trees \\ and Citrus, Via di Fioranello, 52,00134 Roma, Italy. E-mail: \\ valeria.scala@crea.gov.it
}

Xylella fastidiosa is an insect vector-transmitted bacterial plant pathogen associated with severe diseases in a wide range of plants. In the last decade, $X$. fastidiosa was reported in European Community: Italy, France, Spain, Portugal, The Netherlands, Germany and Israel. In Italy, controls are in place to prevent the bacterium from spreading. Among $X$. fastidiosa subspecies this research study was focused on $X$. fastidiosa subsp. pauca ( $X f p$ ) associated to the Olive Quick Decline Syndrome causing severe losses in Salento (Apulia region, southern Italy). A metabolomic approach by LC-TOF and LC-MS/ MS was used to analyse the lipids profile of naturally infected and uninfected olive trees. Sixty samples (xylem tissue, from 1- or 2year-old twigs) of Olea europaea (cv. Ogliarola salentina) were collected in groves located in Copertino (Lecce province) and in Grottaglie (Taranto province). The untargeted profile highlighted that lipids entities significantly clustered the infected samples apart from the healthy ones. In the negative ion modality, we identified $\sim 400$ compounds differently accumulated. HPLC-MS/MS targeted methods was used to characterize and quantify different lipid entities amongst the group of previously identified compounds. By a chemometric analysis, we created a shortlist of 13 compounds significantly different in the infected versus healthy samples. These compounds were added in the liquid culture media to determine their effect on Xfp growth and biofilm formation. The selected compounds affected both process in Xfp, confirming the role of the lipids as signal molecules to arrange the bacteria cell lifestyle (i.e. twitching motility and biofilm formation).

Funding information: Regione Puglia thought the agreement "Strategie di controllo integrato per il contenimento di Xylella fastidiosa in oliveti pugliesi ed analisi epidemiologiche del complesso del disseccamento rapido dell'olivo (CoDiRO; Italian Ministry of Agriculture, Food Forestry and Turism Policies Salvaguardia e valorizzazione del patrimonio olivicoloitaliano con azioni di ricerca nel settore della difesa fitosanitaria (SALVAOLIVI)" and Olivicoltura e difesa da Xylella fastidiosa e da insetti vettori in Italia (Oli.Di.X.I.It)

\section{The role of micronutrients and phenolic compounds in the infection and control of Xylella fastidiosa subsp. pauca in olive trees}

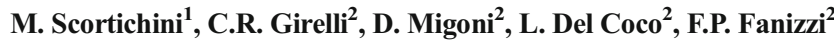

${ }^{1}$ Consiglio per ricerca in agricoltura e l'analisi dell'economia agrariaCentro di ricerca per l'Olivicoltura, Frutticoltura e Agrumicoltura, Via di Fioranello, 52; 00134 Roma; ${ }^{2}$ Dipartimento di Scienze e tecnologie Biologiche ed Ambientali-Centro Ecotekne, Università del Salento, S.P. 6 Lecce-Monteroni.E-mail: marco.scortichini@crea.gov.it
} 
In the different Xylella fastidiosa-plants pathosystems, micronutrients and phenolic compounds are involved in the virulence of the bacterium and in the crop defense system, respectively. Recent studies revealed that also for $X$. $f$. subsp. pauca-olive pathosystem, some micronutrients and phenolic compounds play important roles either in the infection process or in the pathogen control. Through inductively coupled plasma atomic emission spectroscopy (ICP-AES), a low content of manganese, molybdenum and boron was found in soils of Gallipoli area where outbreaks of the disease were firstly observed. A low copper and molybdenum availability was consistently found in the leaves of infected olive trees in different locations of the infected area (Lecce province). Together with zinc and above $200 \mu \mathrm{M}$, copper ion inhibits biofilm formation by $X$. fastidiosa within the xylem vessels, and an in planta detoxification of such ions is required for the bacterium multiplication. The management of such ions within the xylem network could have potential benefit for the field control of disease. Phenolic compounds were consistently found associated in different olive cultivars naturally-infected by $X$. $f$. subsp. pauca. However, solely quinic acid, a precursor of lignin that relevantly accumulates during the infection, would seem a biomarker for the infection, as revealed by ${ }^{1} \mathrm{H}-\mathrm{NMR}$ metabolite fingerprinting and multivariate statisti$\mathrm{cal}$ analyses. These compounds are putatively involved in the plant defense mechanisms even though in the $X . f$. subsp. pauca-olive pathosystem, if the cell density of the pathogen is not lowered by effective bactericidal compounds, the infection can kill the tree in some years.

\section{Use of biocontrol agents to control Phytium ultimum and Rhizoctonia solani and their effects on plant root microbi- ota}

G.R. Shahzad ${ }^{1}$, A. Passera ${ }^{1}$, V. Vacchini $^{2}$, G. Cocetta ${ }^{1}$, A. Ferrante ${ }^{1}$, L. Piazza ${ }^{2}$, P. Casati ${ }^{1}$

${ }^{1}$ Department of Agricultural and Environmental Sciences, University of Milano, Via Celoria 2, 20133 Milano, Italy; ${ }^{2}$ Department of Environmental Science and Policies, University of Milano, Via Celoria 2, 20133 Milano, Italy.E-mail: paola.casati@unimi.it

Pythium ultimum and Rhizoctonia solani are two of the most destructive soil-borne pathogens of Romaine lettuce (Lactuca sativa L. var. longifolia). Protecting the lettuce from these pathogens without losing its nutraceutical values are the growing challenges for Nutrition Sensitive Agriculture (NSA). Application of antagonists as biological control agents can be an alternative without compromising health-related properties, sustainable production and maintaining biodiversity in the agroecosystem. In this study, we tested two endophytic bacteria Paenibacillus pasadenesis 'R16' and Bacillus amyloliquefaciens ' $\mathrm{CC} 2$ ' to evaluate their biocontrol efficacy, and the changes they induced in the microbial diversity inside root tissues, plants' photosynthetic efficiency, and nutraceutical quality parameters. Strain R16 significantly reduced the symptom severity against both pathogens, while $\mathrm{CC} 2$ gave a significant reduction only against $R$. solani. Higher seed germination rate was observed among Rhizoctonia infected plants treated with R16, while CC2 strain showed higher rate in uninfected plants depicting plant growth promotion. A PCA analysis of bacterial community data identified three separate clusters, as the treatments segregate from one another. Both strains stimulated a change in the microbiota but no direct colonization in roots was found. $\mathrm{CC} 2$ reduced the overall diversity, suggesting no detrimental effect on the plant, but, on the contrary, supported a healthier phenotype. Furthermore, higher level of total chlorophyll content supported the efficacy of R16 among treated and infected leaves displaying positive physiological effect on carbon fixation.

Research supported by the project "Difes a fitosanitaria sostenibile per un programma agro-alimentare nutrition sensitive" funded by the Italian Ministry of Health.

\section{An innovative solarization system working like a "solar hot panel" to control soil-borne pathogens}

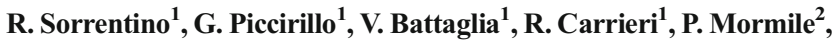 \\ M. Cermola ${ }^{1}$, E. Lahoz ${ }^{1}$ \\ ${ }^{1}$ CREA-Council for Agricultural Research and Economics - Research \\ Centre for Cereal and Industrial Crops, Via Torino 2, 81100 Caserta, \\ Italy; ${ }^{2}$ Institute of Applied Sciences and Intelligent Systems of CNR, Via \\ Campi Flegrei 34, 80072 Pozzuoli (Naples), Italy. E-mail: \\ ernesto.lahoz@crea.gov.it
}

Soil-borne fungal diseases are among the major problems in agricultural crops and actual strategies to contain them rely on the use of ecofriendly approaches such as developing alternative inputs to synthetic chemicals. The solarization is a non-chemical practice aimed to reduce the use of agrochemicals and the fungal inoculum in the soil. An innovative soil solarization system ( $\mathrm{InnS}$ ) working like a solar hot panel has been tested over two years in Sele valley in combination with the addition to the soil of compost. In 2014, InnS and standard solarization method (SFS) significantly lowered viability percentage of propagules of three fungi (Fusarium oxysporum f. sp. melonis, Sclerotinia sclerotiorium and Plectosphaerella cucumerina) compared to the untreated control. Indeed, viability of $F$. oxysporum propagules, as average of three soil depths, was significantly reduced by $80 \%$ in InnS compared to SFS, whereas $S$. sclerotiorum and P. cucumerina viability were reduced by $60 \%$ and $73 \%$, respectively. In 2016, InnS and SFS treatments showed a similar efficacy in the control of $F$. oxysporum and P. cucumerina, while InnS was more effective in the control of $S$. sclerotiorum reducing viability of propagules of $54 \%$. This method is more effective than the traditional one to reduce the inoculum of three important soil-borne fungal pathogens, particularly at $30 \mathrm{~cm}$ depth in the soil. These results were due to a major heat accumulation in InnS which overcomes the well-known limitation of soil heating at major depths of standard solarization. This approach might be associated to other good practices to contribute to the management of these pathogens.

This work was supported by MIPAAF, Project MABISCOMBI, Decree of Ministry n.15532/7818/2013 on 07/31/2013

\section{First report and partial characterization of saffron latent virus infecting saffron (Crocus sativus $\mathbf{L}$.) in Italy}

\section{A. Taglienti, S. Bertin, L. Tomassoli}

CREA Research Centre for Plant Protection and Certification, Via C.G. Bertero 22 - 00156 Rome, Italy. E-mail: anna.taglienti@crea.gov.it

Saffron (Crocus sativus L.) is a minor crop in Italy but with a high added value, being the most expensive spice grown in the world. In November 2018, a field survey was carried out for pathogen investigation in two saffron cropping areas of Latium (central Italy). Plants showing yellowing and stunting and asymptomatic plants were collected (total 45 samples). Potyviruses cause symptomatic and asymptomatic infections in saffron; genus-specific RT-PCR 
indicated the presence of potyvirus in $48 \%$ of the samples. Speciesspecific RT-PCR were positive for bean common mosaic virus (BCMV) and negative for bean yellow mosaic virus (BYMV) and turnip mosaic virus (TuMV). The BCMV sequenced amplicons had $86 \%$ identity with saffron latent virus (SaLV) (isolate Ir-Kh1 from Iran, accession number KY562565). Based on species demarcation criteria for Potyviridae, in $2018 \mathrm{SaLV}$ was released by the International Committee on Taxonomy of Viruses (ICTV) as a new Potyvirus species belonging to BCMV subgroup. The presence of SaLV in BCMV-positive samples was confirmed by RT-PCR using SaLV-specific primers targeting the coat protein $(\mathrm{CP})$ gene. The complete CP sequences had $85 \%$ identity with isolate Ir-Kh1. A phylogenetic tree was built, showing Italian SaLV isolates clustered with Iranian isolate in the potyviruses BCMV subgroup. Genetic variability between Italian and Iranian isolates was analyzed, obtaining a significant nucleotide diversity $\left(\mathrm{P}_{\mathrm{i}}=0.191\right)$. Bio-assays were performed by mechanical inoculation of SaLVinfected sap on Chenopodium amaranticolor; all inoculated plants developed chlorotic local leaf lesions at 5-7 dpi, and RT-PCR confirmed the presence of SaLV in the inoculated leaves.

\section{Insights on grapevine Pinot gris disease: development of viral infectious clones to study virus-host interaction}

\section{G. Tarquini $^{1}$, G. Zaina $^{1}$, P. Ermacora ${ }^{1}$, F. De Amicis ${ }^{2}$, B. Franco- Orozco $^{1}$, N. Loi ${ }^{1}$, M. Martini ${ }^{1}$, G.L. Bianchi ${ }^{2}$, L. Pagliari ${ }^{1}$, G. Firrao $^{1}$, E. de Paoli ${ }^{1}$, R. Musetti ${ }^{1}$}

${ }^{1}$ Department of Agriculture, Food, Environmental and Animal Sciences, University of Udine, via delle Scienze, 206, I-33100 Udine, Italy; ${ }^{2}$ ERSA, Plant Protection Service. Via Sabbatini 5, 33050, Pozzuolo del Friuli (UD), Italy.E-mail: giulia.tarquini@uniud.it

The grapevine Pinot gris disease (GPG-d) is a novel disease characterized by symptoms such as mottling and leaf deformation, which has been recently reported in grapevines, and mostly in Pinot gris. Plants show obvious symptoms at the beginning of the growing season, while in summer symptom recovery frequently occurs, manifesting as symptomless leaves. A new Trichovirus, named grapevine Pinot gris virus (GPGV), belonging to Betaflexiviridae family was found in association with infected grapevines. The detection of the virus also in asymptomatic plants raised doubts about disease aetiology. The primary target of this work was to develop a reliable system for the study of the disease in controlled conditions, avoiding interfering factor(s) that could affect symptom occurrence. To this end, two clones of the virus, pRI::GPGV-vir and pRI::GPGV-lat, were generated from total RNA collected from one symptomatic and one asymptomatic Pinot gris grapevine, respectively. The clones, which encompassed the entire genome of the virus, were used in Agrobacterium-mediated inoculation of Vitis vinifera and Nicotiana benthamiana plants. All inoculated plants showed symptoms regardless of their inoculum source, demonstrating a correlation between the presence of GPGV and symptomatic manifestations. Four months post inoculum, the grapevines inoculated with the pRI::GPGV-lat clone developed asymptomatic leaves that were still positive to GPGV detection. Three to four weeks later, the same phenomenon was observed in the grapevines inoculated with pRI::GPGV-vir. This observation perfectly matches symptom progression in infected fieldgrown grapevines, suggesting a possible role for plant antiviral mechanisms, such as RNA silencing, in the recovery process.

The work has been funded by Regione Friuli-Venezia Giulia (Italy) CUP: F22I15000110002.
The Fusarium graminearum FGSG_03624 xylanase primes plant immune responses and increases plant disease resistance

\author{
S. Tundo ${ }^{1}$, M.C. Paccanaro ${ }^{1}$, I. Moscetti ${ }^{2}$, R. D'Ovidio ${ }^{3}$, F. Faoro ${ }^{4}$, F. \\ Favaron $^{1}$, L. Sella ${ }^{1}$
}

${ }^{1}$ Department of Land, Environment, Agriculture and Forestry (TESAF), University of Padova, Viale dell'Università 16, 35020 Legnaro (PD), Italy; ${ }^{2}$ Department of Ecology and Biology (DEB), University of Tuscia, Via S. Camillo de Lellis snc, 01100 Viterbo, Italy; ${ }^{3}$ Department of Agricultural and Forestry Sciences (DAFNE), University of Tuscia, Via S. Camillo de Lellis snc, 01100 Viterbo, Italy; ${ }^{4}$ Department of Agricultural and Environmental Sciences, University of Milano, Via Celoria 2, 20133 Milano, Italy.E-mail: silvio.tundo@unipd.it

Plants activate defense responses against pathogens once pattern recognition receptors (PRRs) perceive the presence of pathogen-associated molecular patterns (PAMPs). Some cell wall degradating enzymes function as PAMPs and activate the plant immune responses, as shown for EIX and Xyn11A xylanases of Trichoderma viride and Botrytis cinerea, respectively. Since the Fusarium graminearum FGSG_03624 xylanase has been shown to induce necrosis and hydrogen peroxide accumulation in wheat independently from its enzymatic activity, we investigated its ability to modulate plant immunity. To this aim, we transiently and constitutively expressed an enzymatically inactivated form of FGSG_03624 in tobacco and Arabidopsis, respectively. Afterwards, these plants were challenged with Pseudomonas syringae pv. maculicola and B. cinerea. Effectiveness in reducing symptoms caused by the bacterial pathogen was evident both in Arabidopsis and in tobacco whilst no symptoms reduction was observed after $B$. cinerea infection. The increased resistance of Arabidopsis plants to $P$. syringae was associated to a faster and stronger activation of jasmonate/ethylene and salicylate pathways detected after pathogen inoculation. The priming effect was also confirmed after $F$. graminearum inoculation of durum wheat spikes exogenously treated with the FGSG_03624 xylanase. Indeed, wheat spikes treated with the xylanase exhibited reduced symptoms in the early phases of infection and a lower fungal biomass accumulation in semolina compared to control. Besides, callose deposition was detected in infected spikes previously treated with the xylanase and not in infected control plants. In conclusion, our results highlight the ability of FGSG_03624 to prime defense responses in plants thus conferring disease resistance.

\section{Early molecular detection of the bunt disease fungi, Tilletia} caries and T. laevis, in wheat plants

\section{M.T. Valente, L. Orzali, G. Di Giambattista, L. Riccioni}

CREA-DC, Research Centre for Plant Protection and Certification. Email: luca.riccioni@crea.gov.it

Common bunt is an economically important disease of wheat caused in Italy by two fungi, Tilletia caries and T. laevis. The disease has been effectively controlled by seed treatments and use of tolerant/resistant cultivars. Recently, the spread of organic and low-input farming, where no chemical treatments are used and ancient/obsolete species are cultivated, led to the re-emergence of the disease. In this scenario, the development of new tools for screening process in breeding programs or for testing organic seed treatments is required. To this purpose, an accurate method for the early detection of $T$. caries and T. laevis was evaluated in wheat seedling, starting from 30 days following seeding (dfs), before the 
tillering phase. A qualitative and quantitative diagnostic assay was evaluated using a crude extract and a purified DNA, respectively, from a 0.5$1 \mathrm{~cm}$ of the basal shoots $(30,49$ and $79 \mathrm{dfs})$ and from first and second leaves (102 dfs) of naturally infected wheat plants. A TaqMan Real Time PCR assay with species-specific primers and probe was used. The amplification standard curves for both the extracts showed an efficiency $>90 \%$ and $<100 \%$, with a high coefficient of determination $(\mathrm{R} 2>0.99)$ and a high sensitivity (10 fg). The percentages of molecular detection were $83 \%$ at $30 \mathrm{dfs}, 57 \%$ at $49 \mathrm{dfs}$ and $100 \%$ at $79 \mathrm{dfs}$ for shoots; $83 \%$ and $50 \%$ at $102 \mathrm{dfs}$ for first and second leaves of the same plant respectively. Symptom assessment, at maturity stage, will need to confirm the accuracy of the molecular predictions.

Regulation of griseofulvin biosynthesis and role on growth and virulence of Penicillium griseofulvum, causal agent of apple blue mold

S. Valente ${ }^{1,2}$, A. Cometto ${ }^{2}$, G. R. Meloni ${ }^{1,2}$, E. Piombo ${ }^{1,2}$, A.R. Ballester $^{3}$, L. Gonzalez Candelas ${ }^{3}$, M.L. Gullino ${ }^{1,2}$, D. Spadaro ${ }^{1,2}$

${ }^{1}$ AGROINNOVA - Centro di competenza per l'Innovazione in campo agro-ambientale, Largo Paolo Braccini 2, 10095 Grugliasco, Italy; ${ }^{2}$ DISAFA - Dipartimento di Scienze Agrarie, Forestali ed Alimentari, Universitá degli Studi di Torino, Largo Paolo Braccini 2, 10095 Grugliasco, Italy; ${ }^{3}$ IATA-CSIC - Instituto de Agroquímica y Tecnología de Alimentos. Catedrático Agustín Escardino 7, Paterna, 46980Valencia,Spain.E-mail: davide.spadaro@unito.it

Many Penicillium spp. can cause apple blue mold, an important postharvest disease of apple fruits. These species are able to produce many secondary metabolites including mycotoxins on infected fruits, such as patulin and cyclopiazonic acid. Among the casual agents of apple blue mold, $P$. griseofulvum produces huge amount of patulin and a characteristic antifungal compound called griseofulvin. In order to study the role of griseofulvin for the growth and pathogenicity of P. griseofulvum, the regulation of its biosynthesis was investigated. Two approaches were followed: generation of deletion mutants for the putative genes encoding transcription factors in griseofulvin gene cluster and promoter analysis to study the involvement of global regulators. The analyses revealed that $g f R l$ encodes a negative transcription factor of the cluster, which could be involved in the regulation of other genes, such as other secondary metabolites biosynthetic genes and genes involved in the asexual development and virulence of the pathogen. Many stimuli are also important to activate griseofulvin biosynthesis, such as carbon and nitrogen available in the environment, stress response and sporulation. This study reveals that the regulation of griseofulvin involves both a pathway-specific transcription factor and global regulators of gene clusters. Furthermore, the analysis highlights a deep interconnection between secondary metabolism and fungal development.

This work was supported by Fondazione Cassa di Risparmio di Cuneo (progetto SMARTAPPLE - Innovative and SMART technologies for sustainable apple production).

\section{Terpene synthases in Trichoderma gamsii T6085}

I. Vicente ${ }^{1,2}$, R. Baroncelli ${ }^{2}$, M.E. González-Rey ${ }^{3}$, I.G. Collado ${ }^{3}$, R.

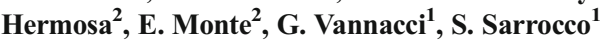

${ }^{1}$ Department of Agriculture, Food and Environment, University of Pisa, Pisa (Italy); ${ }^{2}$ Department of Microbiology and Genetics, SpanishPortuguese Institute for Agricultural Research, University of Salamanca, Villamayor (Spain); ${ }^{3}$ Department of Organic Chemistry, University of Cádiz, Puerto Real (Spain). E-mail: i.vicentemu@gmail.com
Secondary metabolites (SM) play an important role in the interaction between fungi and the environment. The isolate T6085 of Trichoderma gamsii is a promising biocontrol agent, with demonstrated activity against Fusarium graminearum, one of the most aggressive causal agents of Fusarium Head Blight on wheat. The complete genome sequence of $T$. gamsii $\mathrm{T} 6085$ was screened by antiSMASH 5.0 for the identification of the gene clusters putatively involved in SM biosynthesis. The availability of genomic sequences of a large number of Trichoderma species enabled to compare the core-gene content involved in SM biosynthesis among them. Genes encoding terpene synthases (TS) are widely represented in this genus, suggesting an important role in the ecology of Trichoderma. A comparative genomic approach has been carried out in order to characterize TS genes within Trichoderma genus. The genome of T. gamsii T6085 harbours eleven putative terpene synthase-encoding genes, seven of which have been predicted to belong to SM clusters. Interestingly, one of these was predicted to encode a trichodiene synthase (TRI5), which has only been described in the trichothecene-producer Trichoderma species belonging to the Brevicompactum clade. Aimed to decipher the putative function of these genes and to infer their possible role in the interactions of our isolate with the environment, expression patterns of TS genes have been assessed in several induction media and during T. gamsii T6085-wheat and T. gamsii T6085-F. graminearum-wheat interactions. In addition, a preliminary metabolic profile has been determined by HPLC-RMN in 12-day PDB cultures of this Trichoderma strain.

\section{Insight into the citrus Huanglongbing pathosystem and} disease control

\section{N. Wang}

Citrus Research and Education Center, Department of Microbiology and Cell Science, University of Florida/ Institute of Food and Agricultural Sciences, Lake Alfred, FL 33823, USA. Email: nianwang@ufl.edu

Citrus is one of the most important fruit crops worldwide. However, citrus production is facing an unprecedented challenge due to Huanglongbing (HLB, also known as greening). HLB is the most destructive disease of citrus and causes tremendous damage to citrus industry in the world. HLB, caused by the Gram-negative bacterium Candidatus Liberibacter, is widely distributed in the citrus producing regions in Asia, Africa, and Americas. Ca. Liberibacter resides inside the phloem and is vectored by psyllids. All commercial citrus varieties are susceptible to HLB. The understanding of the biology and virulence mechanism of the pathogen remains limited due to the inability to culture the bacteria. Here I will present our current progress in understanding the biology and virulence mechanism of Las, as well as their implications in HLB/ACP management. I will talk about HLB/ACP control strategies based on the epidemiological stages of HLB pathosystem.

\section{Walnut blight causing by endophytic fungi during differ- ent phenological phases of the host}

\author{
A. Zabiák ${ }^{1}$, F. Takács ${ }^{2}$, E. Sándor ${ }^{1}$ \\ ${ }^{1}$ Institute of Food Science, Faculty of Agricultural and Food Sciences and \\ Environmental Management, University of Debrecen, Debrecen, Hungary; \\ ${ }^{2}$ Fruit Research Institute, National Agricultural Research and Innovation \\ Centre, Újfehértó, Hungary. E-mail: toth.andrea@agr.unideb.hu
}


Walnut (Juglans regia) production and export is significant in Hungary. Fruit blight is responsible for great economic losses. It has been reported in many areas of the world, including Hungary. Botryosphaeria and Diaporthe genera are well-known pathogens on different hosts, however fungicide treatment has not been included in the plant protection of walnut yet. Our aims were to identify the fungal pathogens of this destructive disease and determine the peak period of susceptibility of the plant. More than 200 diseased and symptomless walnuts were sampled in North-East Hungary in 2018, and fungi were isolated from the diseased fruits. Cankered shoots as well as symptomless buds were also collected during 2018 and 2019 from the same orchards for the detection and analysis of fungal pathogens. Isolated plant pathogenic fungi were identified based on morphological characters and sequence analysis of ITS and TEF1 regions. Botryosphaeria spp. and Diaporthe spp. were widely distributed in the studied orchards, both in the rotten fruits and on the cankered shoots. The ratio of the two genera isolated from the symptomless buds were proportional to fungi isolated from fruits during the previous autumn. It can be concluded that Botryosphaeria spp. and Diaporthe spp. are the most widespread fungal pathogens causing fruit blight on walnut in North-East Hungary. The pathogens are present on the cankered shoots and in the bud. The pathogens can be transferred by infected pollens or originate from the cankered shoots.

\section{Isolating mycoviruses for a promising biocontrol ap- proach of Fusarium head blight disease}

\section{J. Zhang, M. Calassanzio, A. Prodi, C. Ratti}

Department of Agricultural and Food Sciences, University of Bologna, Viale G. Fanin, 40127, Bologna, Italy.E-mail: claudio.ratti@unibo.it
Fusarium head blight (FHB) is a global wheat disease caused by Fusarium spp., mainly $F$. culmorum and $F$. graminearum which causes severe yield losses and releases mycotoxins in the grains. According to the requirements of sustainable agriculture and food safety, this research aims to identify mycoviruses that are able to infect and confer hypovirulence to species of the genus Fusarium in order to control FHB. By using Next Generation Sequencing (NGS) analyses on 30 F. poae strains, more than 40 putative viral species have been identified belonging to the genera Partitivirus, Betapartitivirus, Victorivirus, Totivirus, Betaendornavirus, Mitovirus, Megabirnavirus, Chrysovirus, Hypovirus, and to the proposed new genera Polymycovirus, Fusaguravirus, and Alternavirus or to unclassified mycoviruses. Some of the detected viruses are similar to the mycovirus from genera that have been studied as inducers of hypovirulence therefore growth rate analysis has been performed on selected fungal strains. In addition, in collaboration with the Nagoya University (Japan), bicistronic, dual-luciferase assay system was developed for the identification of IRESs in the 5' untranslated regions of the genomes of diverse positive-sense single stranded RNA and double stranded RNA. This tool is suitable to understand the initial regulation mechanisms of the translation of viruses in the host, a key step for the interaction virus-fungus.

Publisher's note Springer Nature remains neutral with regard to jurisdictional claims in published maps and institutional affiliations. 